Florida International University FIU Digital Commons

\title{
A Floristic Study of Halmahera, Indonesia Focusing on Palms (Arecaceae) and Their Seed Dispersal
}

Melissa E. Abdo

Florida International University, mabdo002@gmail.com

DOI: $10.25148 /$ etd.FIDC001976

Follow this and additional works at: https://digitalcommons.fiu.edu/etd

Part of the Biodiversity Commons, Botany Commons, Environmental Studies Commons, and the Other Ecology and Evolutionary Biology Commons

\section{Recommended Citation}

Abdo, Melissa E., "A Floristic Study of Halmahera, Indonesia Focusing on Palms (Arecaceae) and Their Seed Dispersal" (2017). FIU Electronic Theses and Dissertations. 3355.

https://digitalcommons.fiu.edu/etd/3355 


\section{FLORIDA INTERNATIONAL UNIVERSITY}

Miami, Florida

\section{A FLORISTIC STUDY OF HALMAHERA, INDONESIA}

FOCUSING ON PALMS (ARECACEAE) AND THEIR SEED DISPERSAL

A dissertation submitted in partial fulfillment of the requirements for the degree of

DOCTOR OF PHILOSOPHY

in

BIOLOGY

by

Melissa E. Abdo 
To: Dean Michael R. Heithaus

College of Arts, Sciences and Education

This dissertation, written by Melissa E. Abdo, and entitled A Floristic Study of Halmahera, Indonesia Focusing on Palms (Arecaceae) and Their Seed Dispersal, having been approved in respect to style and intellectual content, is referred to you for judgment.

We have read this dissertation and recommend that it be approved.

Javier Francisco-Ortega

Joel Heinen

Suzanne Koptur

Scott Zona

Hong Liu, Major Professor

Date of Defense: May 24, 2017

The dissertation of Melissa E. Abdo is approved.

Dean Michael R. Heithaus

College of Arts, Sciences and Education

Andrés G. Gil

Vice President for Research and Economic Development and Dean of the University Graduate School

Florida International University, 2017 
(C) Copyright 2017 by Melissa E. Abdo

All rights reserved. 


\section{ACKNOWLEDGMENTS}

This research was supported in part by the International Center for Tropical Botany (ICTB), Kenari Eco Institute (KEI), Fairchild Tropical Botanic Garden (FTBG), Bogor Botanical Garden of the Indonesian Institute of Sciences (LIPI), and Burung Indonesia. I also thank the Indonesian Ministry of Research and Technology \& the Taman Nasional Aketajawe-Lolobata for assistance with all relevant national and local research permits, and for providing access.

I am grateful for the professionalism of the staff of the Plant Conservation Center of the Bogor Botanical Garden, and especially to Yayan Wahyu Kusuma for his expertise and the extended time in remote forests in the name of research, and to Risna Rosniati for her many contributions in the field as well as her efforts in the herbarium. I am indebted to Burung Indonesia’s Halmahera Team (especially David Purmiasa, Hanom Bashari, Irfan Rosiati) for their extraordinary generosity sharing their expert knowledge, the memorable birding and botanizing outings together come rain or shine, and logistical support at their office and field sites.

I am grateful for the expert capabilities, knowledge, and friendship of Mahroji, whose enthusiasm for biodiversity and conservation leadership is having meaningful impacts on the preservation of Halmahera's forests. I am thankful to the many Indonesian field assistants for their tireless work and to the Halmaheran people for their hospitality and kindness. Thanks to their willingness to welcome strangers and science into their homes and forests, this research was possible. 
I am indebted to all my committee members for their patience, support, and constructive advice throughout this PhD journey. Dr. Joel Heinen was the first professor to help me gain research experience in Indonesia, and he has provided me with guidance for over a decade; his positive impact has helped me to achieve many goals. Dr. Suzanne Koptur has inspired me over the years through her passion for conserving native flora and fauna, and for investigating ecology. Dr. Javier Francisco-Ortega has been a fountain of knowledge on island plants and endemics, always generously sharing his expertise. Dr. Scott Zona's boundless knowledge of the world of palms and tropical plants has benefitted my research, as has his keen willingness to help improve the quality of this dissertation.

I have endless appreciation for Dr. Hong Liu, my major professor and mentor over the years, for her steadfast support during challenging times and her positive encouragement during every research milestone. Her research, spanning from Florida to China and from classical to progressive ecology, has shown me how to push my own boundaries. I am grateful for her integrity, wisdom, energy, and friendship, and am inspired by the scientist and person that she is. 


\author{
ABSTRACT OF THE DISSERTATION \\ A FLORISTIC STUDY OF HALMAHERA, INDONESIA \\ FOCUSING ON PALMS (ARECACEAE) AND THEIR SEED DISPERSAL
}

by

Melissa E. Abdo

Florida International University, 2017

Miami, Florida

3URIHMRUHong Liu, Major Professor

The dispersal services of frugivores affect plant community assembly, persistence, and gene flow in the short-term, and in the long-term are critical to ensuring that tropical trees and palms can regenerate in disturbed areas and can migrate amidst climate change. Halmahera is the largest Moluccan island within the Wallacea biodiversity hotspot, yet data on its plant and animal distributions and interactions are almost null. I studied the tropical trees and palms of Halmahera and their seed dispersal dynamics. Chapter I explores the palms of the Moluccan islands through field-, herbarium-, and literaturebased studies. The results of herbarium specimen collections are presented within a preliminary list of palms for Halmahera's Aketajawe-Lolobata National Park, and contextualized in a review of regional palm biogeography. Expanding beyond the study of one plant family, Chapter II compiles and examines all the tree and palm taxa of the Moluccan islands in order to infer seed dispersal syndromes for each taxon, resulting in an analysis of over 900 taxa. Zoochory was found in $93 \%$ of plant families, and nearly 
$30 \%$ of endemic taxa rely primarily on dispersal by large-bodied frugivores. The role of a hypothesized keystone disperser (the Papuan hornbill, Rhyticeros plicatus ruficollis) is confirmed experimentally to disperse about $10 \%$ of Halmahera's tropical tree and palm taxa. The final chapter encompasses an ex-situ germination trial and a year-long in-situ experimental study that examines the germination and recruitment of three palm species post-ingestion by Papuan hornbills, in four different habitat types representative of Halmahera's landscape. Palms dispersed by hornbills into disturbed habitats and primary forests resulted in enhanced recruitment, although results varied by habitat and species. Hornbills aid both in forest conservation and recovery/assembly after disturbance. The results of this dissertation provide a foundation for further ecological studies and for enhanced conservation of Halmahera island. 


\section{TABLE OF CONTENTS}

CHAPTER

PAGE

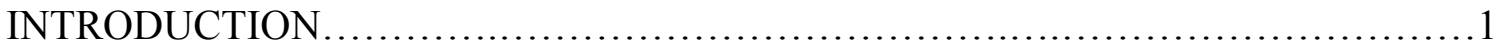



I. THE PALMS (ARECACEAE) OF MALUKU, INDONESIA $\ldots \ldots \ldots \ldots \ldots \ldots \ldots \ldots \ldots$

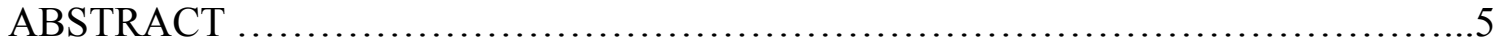

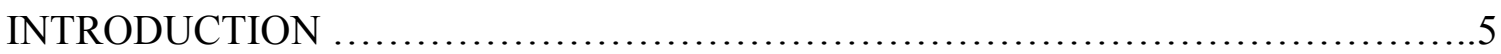

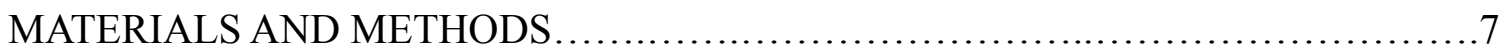

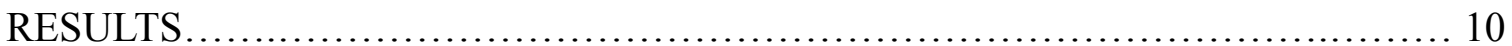

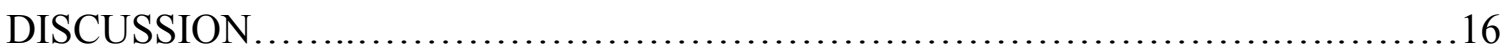

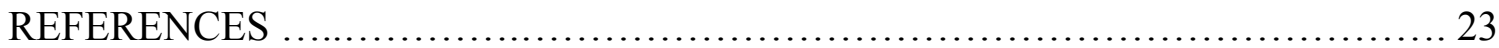

II. SEED DISPERSAL SYNDROMES OF TROPICAL TREES IN WALLACEA, AND AN EXAMINATION OF THE PAPUAN HORNBILL'S ROLE IN SEED

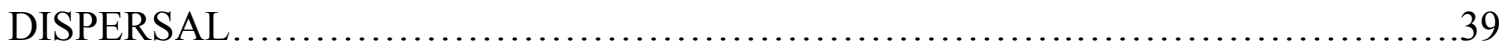

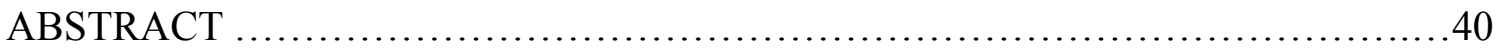

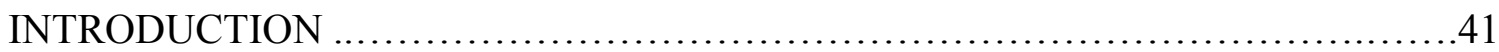



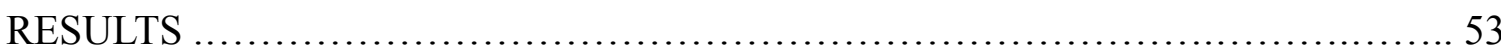

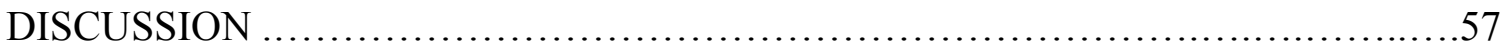

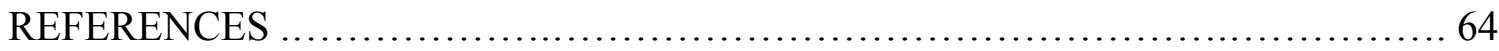

III. PALM (ARECACEAE) SEED DISPERSAL BY THE PAPUAN HORNBILL IN

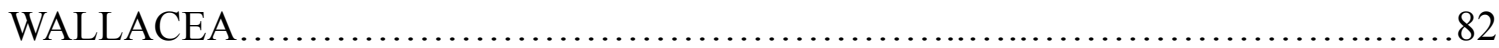

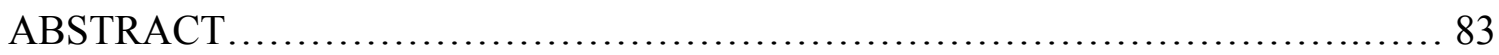

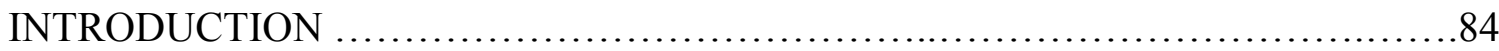

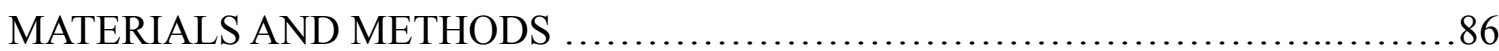

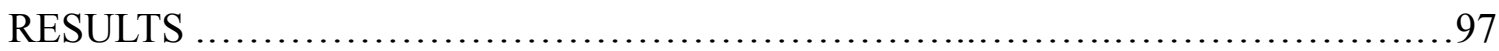

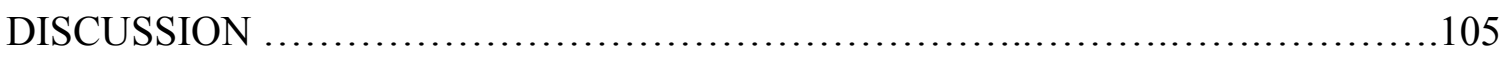

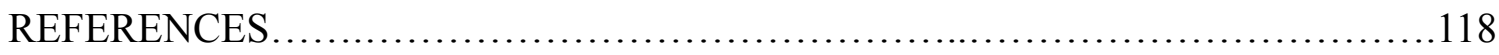

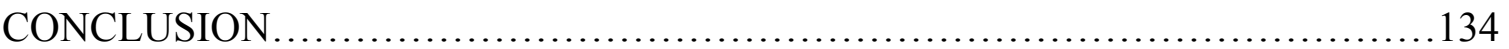

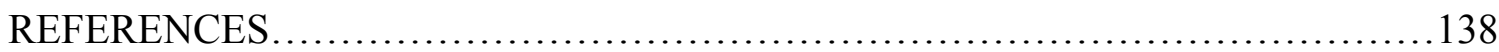

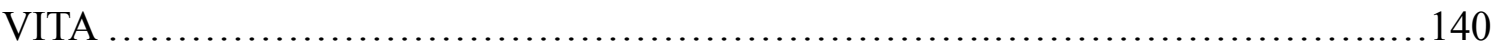




\section{LIST OF TABLES}

TABLE

PAGE

\section{CHAPTER I}

1.1 List of the palms (Arecaceae) of Maluku, Indonesia, noting regional endemism. In "Endemic to Maluku" column: $\mathrm{N}=$ not endemic, $\mathrm{Y}=$ species is endemic

1.2. Summary of the taxonomic diversity of the palms (Arecaceae) of Maluku, Indonesia

1.3. Annotated list of the palms of Aketajawe-Lolobata National Park, Halmahera, Indonesia. In "Presence in Aketajawe-Lolobata NP" column: A= present in Aketajawe block, $\mathrm{L}=$ present in Lolobata block, and A, $\mathrm{L}=$ present in both NP blocks; (s) = a voucher specimen was collected, $(\mathrm{o})=$ an observation of presence was made. In "Endemic to Maluku" column: $\mathrm{N}=$ not endemic, $\mathrm{Y}=$ species is endemic, and $\mathrm{U}=$ unknown status.

1.4. Distribution patterns of palm genera found in Maluku, Indonesia. In "Notes on the distribution..." column: $\mathrm{B}=$ bimodal distribution, with species occurring both East and West of Wallace's Line ("WL"), C= a widespread or cosmopolitan distribution in tropical areas, $\mathrm{E}=$ species occurring primarily East of $\mathrm{WL}, \mathrm{W}=$ species occurring primarily West of WL.......................... 36

\section{CHAPTER II}

2.1. Categories of dispersal syndromes utilized in this study...................76

2.2. Most speciose tree families of the Moluccan Islands of Indonesia. *There were many seed species found in the Papuan hornbill's excrement that were not possible to identify, and may or may not have been from these families. As these families all contain species with zoochorous dispersal, frugivorous birds such as the hornbill could be involved in their seed dispersal.

2.3. Plant species dispersed by the Papuan hornbill, including notation of species utilized in the germination trial. In the "habitat occurrences" column, $1=$ primary forest contiguous \& adjacent to protected forest; $2=$ community agroforest in a mixed-habitat landscape; $3=$ primary forest adjacent to unprotected/mixed use areas. In the "germination trial" column, $U=$ species trialled that did not germinate during study period; $\mathrm{Y}=$ species trialled that did germinate 


\section{CHAPTER III}

3.1. List of palm species ingested by the Papuan hornbill at three study sites in Halmahera island, Indonesia. "Habitat occurrences" are represented by 1: primary forest contiguous with NP; 2 : community agroforest/secondary forest; 3 : primary forest in unprotected mixed habitat matrix......................................133 


\section{LIST OF FIGURES}

FIGURE

PAGE

\section{CHAPTER I}

1.1. Map of Halmahera island highlighting palm study areas, inset within the Wallacea biodiversity hotspot of Indonesia................................... 27

\section{CHAPTER II}

2.1. Map illustrating the Wallacea Biodiversity hotspot containing the Moluccan islands, highlighting Halmahera Island and its Aketajawe-Lolobata National Park.

2.2. A pair of Papuan hornbills (Rhyticeros plicatus ruficollis), frugivores and seed dispersers, in forest habitat of Halmahera, Indonesia.

2.3. Examples of tree fruits with zoochorous dispersal syndromes in the Moluccan Islands of Indonesia........................................... 72

2.4. Proportions of tree species in Moluccan Islands of Indonesia across dispersal syndromes................................................. 73

2.5. Number of plant species ingested by Papuan hornbill at each site in Halmahera............................................................ 74

2.6. Comparison of seed size classes of plant taxa digested by the Papuan hornbill................................................................ 75

\section{CHAPTER III}

3.1. Map illustrating the Wallacea Biodiversity hotspot (Indonesia) containing the Moluccan islands, highlighting Halmahera island and its Aketajawe-Lolobata National Park

3.2. Diagram illustrating planting design of the in-situ palm seed fate experiment carried out at four habitat types on Halmahera island, Indonesia. P1 $=$ palm species $\# 1, \mathrm{~T} 1$ = seed treatment \#1, and so forth for all three palm species and all three treatments. Dashed lines indicate separate zones for each palm species. Dotted lines indicate separate zones for each treatment. Outline

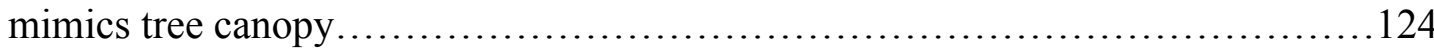


3.3. Comparison of mean germination of three palm (Arecaceae) species experiencing three different diaspore treatments, in a nursery setting...............125

3.4. Germination results of three seed treatments of Pigafetta filaris planted at four different habitat types on Halmahera island, Indonesia.......................126

3.5. Recruitment results of three seed treatments of Pigafetta filaris planted at four different habitat types on Halmahera island, Indonesia.......................127

3.6. Germination results of three seed treatments of Rhopaloblaste ceramica planted at four different habitat types on Halmahera island, Indonesia...............128

3.7. Recruitment results of three seed treatments of Rhopaloblaste ceramica planted at four different habitat types on Halmahera island, Indonesia................129

3.8. Germination results of three seed treatments of Saribus rotundifolius planted at four different habitat types on Halmahera island, Indonesia................130

3.9. Recruitment results of three seed treatments of Saribus rotundifolius planted at four different habitat types on Halmahera island, Indonesia............... 131

3.10. Habitats of Halmahera, Indonesia, including habitats studied over the course of my dissertation and used in the in-situ experiment on seed fates in three native palm (Arecaceae) species. 


\section{INTRODUCTION}

The Wallacea biodiversity hotspot (Myers et al. 2000) is known for its floristic and faunal diversity, but information on the biodiversity and ecology of the Moluccan islands of Wallacea is sparse. The overall goal of my dissertation is to gather and explore data on the dispersal ecology of tropical trees, with a focus on palms (Arecaceae), and connect research findings to the local conservation context in Wallacea. Palms are iconic components of the region's tropical ecosystems, and the Papuan hornbill (Rhyticeros plicatus ruficollis) was identified as a hypothesized keystone disperser of tropical trees. A combination of studies focusing on natural history, field exploration, and ecological experimentation were carried out as part of the dissertation research. Results of these studies have provided insights on the distribution and biogeography of Moluccan palms, the dispersal syndromes of Moluccan plants, the diversity of plants dispersed by Papuan hornbills, and the importance of frugivores' seed dispersal to the assembly and recovery of current and future forests in Wallacea.

Chapter I explores the palms of the Moluccan islands through field-, herbarium-, and literature- based studies. The palm flora of the Moluccan islands was documented in the extraordinary early works of Rumphius (1741), but recently a new monotypic genus of palms, Jailoloa (Heatubun) Heatubun \& WJ Baker, was discovered in Maluku's largest island, Halmahera (Heatubun et al. 2014). Therefore, undertaking a detailed review of the diversity and biogeography of the palms of Maluku was a critical first step to supporting ecological studies of palm dispersal. The results of herbarium specimen 
collections are presented within a preliminary list of palms for Halmahera's AketajaweLolobata National Park, and contextualized in a review of regional palm biogeography.

Going beyond the study of one plant family, in Chapter II I compile and examine all the tree and palm taxa of the Moluccan islands in order to infer seed dispersal syndromes for each taxon. Through analyses of over 900 taxa, I summarize the region's floristic diversity and the dispersal modes of regional trees and palms. A field study and germination trial are employed to test whether species inferred to have zoochorous dispersal syndromes are indeed dispersed by a frugivore (the Papuan hornbill), and to learn whether 35 plant species dispersed by Papuan hornbills are able to germinate successfully.

Chapter III focuses on the dispersal of three different palm species by Papuan hornbills. Specifically, I aimed to discover how dispersal by hornbills and varied habitat types influence the early life history stages of three palms: Pigafetta filaris, Rhopaloblaste ceramica, and Saribus rotundifolius. Although palms are well-known to rely on the dispersal services of frugivores (Zona and Henderson 1989), to date, little is known about hornbills' role in dispersing palm seeds. Information on the fate of hornbill-dispersed seeds in habitats outside of roost and nest sites is also lacking (Kitamura 2011). My study first describes a comparative shade house germination experiment. Then a yearlong field study in four different habitat types provides insights into how dispersal (ingestion) by hornbills, seed species, and habitat affect the germination and recruitment of palms. 
In all chapters, conservation recommendations and future research directions relevant to the local context are provided.

\section{REFERENCES}

Heatubun, C.D., Zona, S., and Baker, W.J. (2014). Three new genera of arecoid palm (Arecaceae) from eastern Malesia. Kew Bulletin 69: 9525.

Kitamura, S. (2011). Characteristics of hornbill-dispersed fruits in lowland dipterocarp forests of southern thailand. The Raffles Bulletin of Zoology, (24), 137-147.

Myers, N., Mittermeier, R. A., Mittermeier, C. G., Da Fonseca, G. A., \& Kent, J. (2000). Biodiversity hotspots for conservation priorities. Nature, 403 (6772), 853-858.

Rumphius, G.E. (1741). Herbarium Amboinense, Netherlands.

Zona, S., and Henderson, A. (1989). A review of animal-mediated seed dispersal of palms. Selbyana 11: 6-21. 


\section{CHAPTER I}

THE PALMS (ARECACEAE) OF MALUKU, INDONESIA 


\begin{abstract}
The Wallacea biodiversity hotspot is known for its floristic diversity, and palms (Arecaceae) are iconic components of the region's tropical ecosystems. On a global level palms are one of the most comprehensively studied plant families. However, there remains a paucity of detailed information on palms at local scales in some tropical areas such as this study's focal area: the Moluccan islands of Indonesia, which lie within the Wallacea biodiversity hotspot. Local-scale information is critical to conservation efforts that are often managed by local practitioners. My study had two related objectives. One objective was to carry out fieldwork, and prepare herbarium specimen collections of palms in Halmahera, the largest Moluccan island. The second objective of this study was to compile a review of Moluccan palms using literature and herbarium resources. Results indicate that nearly half of the palms of Maluku are endemic to the region, and $36 \%$ of the endemic species are conserved within Halmahera's only National Park (NP). Nearly half of all forty-seven palms native to Maluku are present within the NP. The diversity and biogeography of the palms that occur in Maluku are presented and discussed.
\end{abstract}

\title{
INTRODUCTION
}

The overall goal of the present dissertation is to gather and explore data on the dispersal ecology of tropical trees and palms and connect research findings to the local conservation context in Wallacea. The palm (Arecaceae) flora of the Moluccan islands was documented in the extraordinary early works of Rumphius (1741), yet only three years ago a new monotypic genus of palms, Jailoloa (Heatubun) Heatubun \& WJ Baker, 
was discovered in Maluku's largest island, Halmahera (Heatubun et al. 2014). Therefore, undertaking a detailed review of the diversity and biogeography of the palms of Maluku was a critical first step to supporting ecological studies of palm dispersal. To this end, the first objective of this study was to review the palm diversity of the Moluccan islands and examine its regional biogeography. The other objective was to study the palms of Halmahera firsthand and conduct herbarium specimen collection accordingly.

The Wallacea biodiversity hotspot (Myers et al. 2000) is known for its floristic and faunal diversity, and palms are iconic components of tropical ecosystems of Wallacea and almost every tropical site. Situated within the world's largest island archipelago (Indonesia), Wallacea consists of numerous islands (an exact count is elusive). The review focuses upon one of the biogeographic regions within Wallacea: the Moluccan islands, hereinafter referred to as Maluku, following Vane-Wright et al. (1994). The establishment in 2004 of the Aketajawe-Lolobata National Park ("NP”) on Halmahera island resulted in the protection of an estimated $9.4 \%$ of the island's terrestrial landscape. However, even protected areas such as the NP face threats of illegal encroachment and logging (M. Abdo, unpublished data), and unprotected forests are threatened by mining and conversion to agriculture or plantations. There is an urgent need in Maluku for conservation-relevant information to help mitigate these threats to biodiversity.

In this context of opportunities and challenges facing forest conservation in Halmahera, there exists a paucity of information available to local conservation practitioners and NP staff on how they may support species-level conservation of local flora and fauna. Such 
fine-grain information that allows for detailed monitoring of ecological communities is valuable as it can extend benefits not only to in-situ NP conservation but also to ex-situ conservation and restoration of biodiversity.

\section{MATERIALS AND METHODS}

\section{Study Site}

Three biogeographical regions are delineated within Wallacea: the Lesser Sundas, Maluku, and Sulawesi (Vane-Wright et al. 1994). The biogeographical region of Maluku mostly encompasses the Indonesian geopolitical provinces of "North Maluku" and "Maluku," except most workers agree that it does not include the islands in the Sula or Banda Arc chains (e.g., de Jong 1998, Vane-Wright and Peggie 1994). The major island groups within the biogeographical delimitation used for Maluku herein are Halmahera and surrounds (e.g., Bacan, Morotai, Obi, Ternate, Tidore), Seram, Buru, Ambon, and Kai. Many of the islands of Maluku are comprised of fragments of continental crusts, namely Seram, Buru, Obi, Bacan, and Ambon (Monk et al. 1997). Geologically interesting composite islands (islands that are formed from at least two different origins) occur frequently in northern Maluku, such as Halmahera, Morotai, Makian, and Ternate. Maluku is estimated to retain Wallacea's highest overall forest cover, with varied forest ecosystems encompassing about $63 \%$ of Maluku's terrestrial landscape (unpublished data, Indonesian Ministry of Forestry 2011). Native forests have a range of habitats within them and in contrast to some of the forests found west of Wallacea (e.g., Bornean forests), forests in Maluku do not typically have one dominant tree family (e.g., 
Dipterocarpaceae) providing the canopy cover. Many of the forests in Maluku are lowland evergreen forests, especially on Halmahera and adjacent islands where topography is generally low or hilly in elevation (with hills often being between $400 \mathrm{~m}$ $800 \mathrm{~m}$ in elevation). However, many islands in Maluku do have tropical montane forests, especially on the slopes of mountains of volcanic origin and mostly above the elevation of $1000 \mathrm{~m}$, although several instances of montane forest have been found at lower elevations, i.e. $850 \mathrm{~m}$, in Maluku (M. Abdo, unpublished data). Forest habitats on limestone (karst) geologies and on ultrabasic (serpentine) geologies also occur in the region. Notable examples of karst and ultrabasic forests can be found in central and east Halmahera, areas that are also threatened by mining for gold and nickel.

\section{Study species}

Malesia (from peninsular Malaysia south and east into Papua New Guinea) has a palm flora that is estimated to contain about $42 \%$ of the total species diversity found within the family (Dransfield et al. 2008). Within Malesia, the palm flora of Maluku was first studied in depth by Rumphius (1741). Palms are found in a range of habitats in Maluku, from sea level and alluvial zones upwards through a range of elevations to at least $900 \mathrm{~m}$ in elevation (M. Abdo, pers. obs.) except for tall mountains and volcano peaks where the flora is typically composed of perennial herbs, grasses, and shrubs associated with scrub communities (M. Abdo, pers. obs.). Palms are common components of forest communities in Maluku (M. Abdo, unpublished data) yet species diversity of palms in Wallacea is lower than that of bordering areas such as Borneo, western Malesia, and 
Papua New Guinea (Baker and Couvreur 2012). Regardless, significant discoveries continue to be made in Maluku, including the discovery of a new monotypic palm genus (Jailoloa) in Halmahera (Heatubun et al. 2014).

\section{Field study \& literature review of Moluccan palms}

A field study to observe and document fertile palms occurring on Halmahera island was undertaken, as was a detailed review of relevant literature- and herbarium-based information on palms of Maluku. The field studies during which palm specimens were collected were conducted in several primary and secondary forest areas of Halmahera including both the Aketajawe and Lolobata blocks of the NP (Figure 1). On the basis of recommendations from an authority on palms, surveys were designed to encompass habitats that varied in their geological substrates and elevation (S. Zona, pers. comm.) and were carried out over the course of nearly fourteen non-consecutive months in order to account for variation in fruiting and flowering times of palm species. Surveys were conducted by walking along 15 belt transects approximately $1000 \mathrm{~m}$ by $5 \mathrm{~m}$ within each NP block and complemented by additional walks along riverbeds/forest edges to search for palms. Because of the inaccessibility of most forested areas on Halmahera (i.e. lack of trails, boardwalks, or footpaths and terrain that is difficult to traverse) local field guides indigenous to the study areas provided guidance in navigating within the study sites. Herbarium specimens of fertile palms and other vascular plants were collected (in duplicate/triplicate when possible) and deposited in the herbaria of Bogor Botanic Garden, BO, and a NP field station. Estimates of the frequency of occurrence of all palm 
species observed in the NP were made using three categories: rare, occasional, or common. Species were considered rare if fewer than ten individuals were counted in observed subpopulations numbering fewer than five. Species were considered common if they were among the dominant or most abundant species (on the basis of visual estimate) found in more than one habitat type, and were observed consistently throughout the majority $(>80 \%)$ of areas visited within a given Park block. Species were considered occasional if they did not qualify as rare and they were not among the most abundant species in any observed habitat type, and were observed in fewer than $80 \%$ of the areas visited within a given Park block.

The World Checklist on Selected Plant Families was used as a primary source for populating the list of palms extant in Maluku (Govaerts et al. 2016). Collections of palms made in Maluku were consulted in the physical and/or digital herbaria of BO, SING, $\mathrm{K}$, and $\mathrm{L}$. The resulting data were compiled in a database on the palms of Maluku in order to facilitate analysis. Additional literature review was conducted in order to gain insights into the drivers that affect palm biogeography in Maluku, and to summarize the taxonomic diversity of the palms of Maluku.

\section{RESULTS}

\section{Palms of Maluku}

There are twenty-two genera of palms in Maluku that contain a total of forty-seven palm taxa (Table 1). Nearly half of the palms (47\%) of Maluku are endemic to the region: 
twenty-two palm taxa have been recorded only on islands within Maluku. As with other areas in Indonesia and throughout Malesia, most of the palm diversity of Maluku is found in the tribes Areceae and Calameae (Govaerts et al. 2016); combined they contain about $77 \%$ of the species diversity of Maluku (Table 2). All of these species are considered native to Maluku. Cocos nucifera L. is widespread in tropical regions and of uncertain geographic origin (Baker and Couvreur 2012) and therefore not included in this analysis, although it is abundant in Maluku and cultivated widely as a major staple of livelihoods in Halmahera and other Moluccan islands (M. Abdo pers. obs.).

Field study of palms in Halmahera and in Aketajawe-Lolobata National Park resulted in a preliminary assessment of the palms of the NP (Table 3) that includes notes on each species' observed habitats and frequencies within the NP. All palms that were observed in the NP fell into three frequency categories (rare, occasional, or common) and frequencies were noted in the preliminary list (Table 3) in order to aid continued study and conservation of the Park's palms. Twenty-four palm collections were made during the course of fieldwork in Halmahera, representing thirteen palm species. Several of these collections are noteworthy in that they represent species that are poorly known in the wild, and are discussed later in this chapter. Many of the palms in the NP were observed in only one of the two disjunct Park blocks, with six species found only in Aketajawe and six other palm species found only in Lolobata. Further study is needed to determine if these disjunct occurrences are indicative of incomplete field study, variation 
in habitat/community, or are biogeographic in origin. Possible explanations for the disjunct distributions are mentioned in the discussion section of this paper.

\section{Arecoideae}

The palm subfamily Arecoideae is the most diverse globally (Dransfield et al. 2008), but in Maluku it is the second most speciose subfamily with eighteen taxa. Except for Orania palindan, which is in the Oranieae tribe and ranges from the Philippines to N. New Guinea, all Moluccan Arecoideae taxa fall within tribe Areceae. Moluccan taxa of this subfamily are all characterized by having pinnate leaves and flowers arranged in groups of three composed of one pistillate and two staminate flowers (Dransfield et al. 2008).

There are five species of palms in subtribe Arecinae, the most well-known of which is the betel palm, Areca catechu, which is also the subtribe's most widely distributed palm ranging from Bangladesh through to the Solomon Islands (Govaerts et al. 2016). The broad distribution of Areca catechu is a consequence of it being cultivated by humans throughout South and Southeast Asia (Rumphius 2011, Baker and Couvreur 2012). Except for one taxon, the remainder of Arecinae in Maluku range from Sulawesi to New Guinea, and include Areca vestiaria, A. macrocalyx, and Pinanga rumphiana. The one exception is a taxon endemic to Maluku, Pinanga globulifera. Pinanga globulifera was first discussed by Rumphius who called it Pinanga oryzae-formis (Rumphius 2011).

Two subtribes contain only one taxon each in Maluku. The only Moluccan taxon in the Linospadicinae is Calyptrocalyx spicatus, which is also endemic to the region. The 
species was discussed by Rumphius under the name Pinanga globosa, because of its musket-shaped fruits, whose observation that it was found only in mountainous forested areas (Rumphius 2011) holds true today (at least for the species as observed in Halmahera during field study). Subtribe Iguanurinae also has one taxon, Rhopaloblaste ceramica, native to both Maluku and New Guinea; this genus has a remarkably disjunct distribution (Banka and Baker 2004) discussed later in the chapter.

Subtribe Ptychospermatinae is represented by four species in Maluku, three of which are found in both Maluku and New Guinea (Govaerts et al. 2016): Drymophloeus litigiosus, D. oliviformis, and Ptychosperma propinquum. The fourth Ptychospermatinae, Jailoloa halmaherensis, is endemic to the island of Halmahera and was discovered recently and described within a new monotypic genus (Heatubun et al. 2014).

The remaining six species of the Arecoideae are as yet unplaced into subtribes (Baker and Couvreur 2012): Heterospathe elata var. elata, H. glauca, Hydriastele beguinii, $H$. microcarpa, H. moluccana, and H. oxypetala. Except for Heterospathe elata var. elata, the five unplaced Arecoideae are endemic to Maluku (Govaerts et al. 2016) and are poorly known in the wild (Baker and Loo 2004, Gardiner et al. 2012). Three of these poorly known species were found in the wild during the course of field study: Heterospathe glauca, Hydriastele beguinii, and Hydriastele microcarpa.

\section{Calamoideae}

The Calamoideae is the most species rich subfamily in Maluku with nineteen taxa. The Moluccan species in this subfamily are dominated by the climbing palms known as 
rattans, but several Calamoid tree palms also occur in the region (e.g., the Sagu palm and the Pigafetta palm). All Moluccan species in this subfamily are characterized by pinnate leaves, scales covering their fruits, and spines occurring on various parts of their morphology (Dransfield et al. 2008).

Most of the diversity in this subfamily is in subtribe Calaminae, which has fifteen Calamus species: C.albus, C.aruensis, C. beguinii, C.buroensis, C.calapparius, $C$. cawa, C. equestris, C. graminosus, C. halmaherensis, C. heteracanthus, C. niger, $C$. pisicarpus, C. robinsonianus, C. rumphii, and C. validus. Of all Calamus species in Maluku, only $C$. aruensis is a wide-ranging species, extending from the Aru islands to the Solomon islands. Calamus validus and C. robinsonianus occur in both Maluku and Sulawesi, while C. heteracanthus extends from Maluku into Papua (Govaerts et al. 2016). The remaining eleven rattan species of Maluku are endemic to the region, and most are poorly known in the wild.

The remaining four subtribes in the Calamoideae each have only one species that occurs in Maluku. Korthalsiinae is represented by Korthalsia celebica, which is reported only from Sulawesi and the Sula islands of Maluku, although there are unverified reports of this taxon occurring in Halmahera (M. Abdo, pers. obs.). Metroxylon sagu, ranges from Maluku to Papua and is the sole representative of the Metroxylinae subtribe. The sagu palm is important in Maluku (and elsewhere in its range) because of its ecological dominance of many coastal estuaries and inland alluvial habitats in the region, and because of its prominence as a major source of carbohydrates and as a roofing material for local populaces. Pigafetta filaris occurs in Maluku and Papua, and is the only 
representative of subtribe Pigafettinae. Finally, representing subtribe Salaccinae is Salacca zalacca, the wide ranging and often-cultivated salak palm that is believed to have a native range from Sumatra to Maluku.

\section{Coryphoideae}

There are nine species in the subfamily Coryphoideae in Maluku, most of which have rather wide ranges. All Moluccan species in this subfamily are characterized by palmate or pinnate leaves and solitary flowers (Dransfield et al. 2008).

There are three species in the tribe Caryoteae, Arenga microcarpa, $A$. pinnata, and Caryota rumphiana, all with ranges that extend far beyond Maluku.

The sole species in the Corypheae tribe in Maluku is Corypha utan, which also has a very wide range, extending into the Andaman islands and Northern Australia (Govaerts et al. 2016).

Lastly, the Trachycarpeae tribe has five species present in Maluku that all have more limited distributions compared to the other two tribes' species. Three Trachycarpeae are endemic to Maluku: Licuala adscendens, which is known only from a single collection on Obi island (Barfod and Heatubun 2009), Licuala flexuosa, and L. rumphii. Pholidocarpus ihur and Saribus rotundifolius are the other two species in the Trachycarpeae, the former native to Maluku and North Sulawesi, and the latter widespread in Malesia. 


\section{Nypoideae}

The Nypoideae subfamily is unique in having only one palm species within it, Nypa fruticans, a species that is abundant in Maluku. Nypa fruticans has pinnate leaves, a dichotomously branching stem, and is well known for its floating fruit that have aided its dispersal. It is distributed from Sri Lanka to the Caroline Islands (Govaerts et al. 2016).

\section{DISCUSSION}

\section{Conservation of palms within Aketajawe-Lolobata National Park}

On the basis of preliminary studies made in the Park, I estimate that at least twenty-seven species of palms are extant in the NP. Twenty-four species were observed and/or collected and most were identified to species level. Three taxa were Calamus spp. observed in sterile condition and identified only to genus; each of these taxa had vegetative morphologies that appeared distinctive enough to warrant inclusion herein. Eight of the species that occur in the NP are endemic to Maluku, enabling ex-situ conservation of $36 \%$ of the region's endemic taxa to be achieved by the Park. Further study to locate and assess the remaining endemic taxa of Maluku (most of which are Calamus species) and determine if any are extant in the Park is warranted.

The habitats and frequencies of all palm species observed and/or collected as specimens within Aketajawe-Lolobata NP vary, as with other tropical forests in Wallacea and beyond. Of all the palm species observed in the Park, the only palm species that were observed to be markedly rare (fewer than ten individuals in observed subpopulations 
numbering fewer than five) were Calyptrocalyx spicata and Heterospathe glauca. However, many palm species were observed in only one block of the disjunct Park, and not the other. Therefore, a prudent management recommendation is to study the distribution and demography of palms found only in one Park block and monitor them. Given the context of limited research and monitoring resources within the NP, endemic species should be prioritized because endemic species have limited global distributions, and therefore the only opportunity to protect their ex-situ wild populations is likely to be within the Aketajawe-Lolobata National Park.

The palm species that occur within the Park provide food and shelter for, and are dispersed by, frugivorous birds (Monk et al. 1997). Many palms are dispersed by Papuan hornbills, and this subject is covered in detail in the remainder of my dissertation. During the course of fieldwork, I noted any anecdotal observations that I made (i.e. observations outside the formal methodology or experimental scope of my research) that pertained to palm seed dispersal in my field books. Bats (Pteropus spp., Rousettus sp., Nyctimene albiventer, and at least three other unidentified medium-to-large bodied bats) were observed dispersing the seeds of Areca vestiara, Orania palindan, Pinanga rumphiana, Rhopaloblaste ceramica, and Saribus rotundifolius. The dispersal of Orania palindan by bats is particularly interesting because the large size of Orania fruits impedes consumption by many frugivorous birds (Papuan hornbills, for example, were not found to consume this species). Orania palindan was also observed in several instances to recruit successfully to juvenile seedling stage below parent palms (on the basis of 
anecdotal observations of mature Orania palindan palms made during fieldwork). Future study to determine how Orania palindan disperses in the Park would be worthwhile, and potentially indicative of how other "extra large-sized" tree seed species in the park are dispersed. Fruit pigeons were observed feeding on Drymophloeus litigiosus, Pinanga rumphiana, and Hydriastele sp. These observations support the well-accepted notion that frugivores play important roles in the dispersal of palms (Zona and Henderson 1989, Baker and Couvreur 2012).

\section{Diversity, biogeography, and conservation of Moluccan palms}

The most species rich palm genus in Maluku is Calamus (sensu Baker 2015), which has fifteen species in Maluku. The Calamus species encountered during field studies in Halmahera were frequently sterile and therefore a limited number of collections and definitive identifications could be made. Further study focusing solely on this genus in Halmahera (and elsewhere in Maluku) is warranted, especially given that species originally described by Rumphius $(1741,2011)$ are still unknown in the wild and may be extant in Maluku. Although the genus has considerably less diversity in Maluku in comparison with surrounding areas (e.g., Philippines, Papua, and Borneo), recent focal studies on Calamus in adjacent areas (i.e., Sulawesi and Papua) have shown that knowledge of regional Calamus species is inadequate (Powling 2009, Baker and Dransfield 2014). Part of the high diversity of Moluccan Calamus could be explained by the fact that Calamus is among a handful of palm genera that have higher rates of diversification relative to the rest of the Arecaceae (Baker and Couvreur 2013b). 
The next most diverse palm genera in Maluku all have between two and four species that occur in Maluku: Hydriastele, Licuala, Areca, Drymophloeus, Heterospathe, Pinanga, and Arenga. Many of the twenty-two endemic palms of Maluku are found within the most diverse genera; $86 \%$ of them occur in Calamus, Hydriastele, Licuala, and Pinanga alone. Two poorly known endemic Hydriastele species (Baker and Loo 2004) were found extant in Aketajawe-Lolobata NP: Hydriastele microcarpa and H. beguinii.

Heterospathe glauca, another endemic also poorly known in the wild (Gardiner et al. 2012) was found in Tidore, Maluku, within a conservation area. A specimen identified tentatively as a variable phenotype of Heterospathe glauca was also collected within the NP on Halmahera.

In recent years there have been advances in knowledge of global Arecaceae biogeography and distribution (Baker and Couvreur 2013a and 2013b), but in Maluku, island-by-island data on distribution and endemism do not yet exist for vascular plants. In contrast, geological information for Maluku (e.g., Hall 1987, Monk et al. 1997, Suparan et al. 2001) is more plentiful than plant data. The geologic history of the islands in Maluku is complex, and the timeframe during which Halmahera's originating land masses emerged above sea level is unknown (de Jong 1998). The second largest island in Maluku, Seram, is estimated to have emerged five million years ago (Audley-Charles 1993). It is likely that Halmahera collided into its present composite state less than ten million years ago (Hall 1987, Monk et al. 1997). Examination of the pollen record from north Halmahera (within the Kao area) points to the existence of well-established lowland rainforests with 
diverse palm and fern assemblages for at least 3000 years on Halmahera (Suparan et al. 2001). Halmahera and other islands in Maluku contain a diversity of substrates, including alluvial soils, volcaniclastics, volcanic conglomerates, river deltas, ferricrete layers, karst formations, and ultrabasics (Monk et al. 1997, Suparan et al. 2001). Halmahera, while slightly larger (approximately $680 \mathrm{~km}^{2}$ ) in area than Seram, has fewer elevational gradients and probably lower beta-diversity; Halmahera's highest point is $1635 \mathrm{~m}$ and Seram's is $3027 \mathrm{~m}$ (de Jong 1998).

Palms are widespread in many insular ecologies across Malesia (extending from peninsular Malaysia through Indonesia and the Philippines to eastern Papua New Guinea), which has the greatest palm diversity globally (Dransfield et al. 2008). Within Malesia, Wallace's Line (Wallace 1860) provides an accepted biogeographic boundary that is also useful in categorizing varied palm distributions throughout Malesia (Dransfield 1987). As with other Malesian palms, palm genera that occur in Maluku can be categorized into five groups according to the distribution patterns of their phylogenetic lineages. The lineages of Malesian palm genera fall into the following categories that indicate where most of their diversity is present: 1) distributions to the west of Wallace's Line ("WL"); 2) distributions to the east of WL; 3) bicentric distributions; 4) disjunct distributions; and 5) widespread distributions (after Dransfield 1987, Baker and Couvreur 2012).

The most diverse palm genera in Maluku (the eight genera with two or more species that occur in Maluku) have their centers of diversity either east or west of WL, or have 
bicentric distributions (Table 4). Two genera are most diverse east of WL, and one of them, Hydriastele, has speciated within Wallacea (Baker and Couvreur 2012). Three genera have centers of diversity west of WL. Two of these are Areca and Pinanga, which are likely to have diverged and dispersed eastward into Wallacea and Papua after the Sunda and Sahul zones came together during the Miocene epoch (Dransfield 1987, Dransfield et al. 2008). The three remaining most diverse genera of Maluku have bicentric distributions. In the case of Calamus, it is thought that the genus has had a wide range (extending from the Sunda lands through Malesia and into the Pacific) since the Eocene and possibly as early as 65 million years ago (Baker and Couvreur 2013a). Another genus with bicentric distribution, Heterospathe, has high diversity in the Philippines and Papua but is otherwise absent from the far western lands of Malesia, and is believed to have diverged during the early Miocene (Baker and Couvreur 2012).

The varied distributional patterns combined with the geographic spreads of palms across the islands of Maluku are indicative of long-distance dispersal (e.g., spanning oceans) and a variety of dispersal mechanisms and events. (Other factors surrounding speciation and extinction affect palm biogeography but are outside the scope of this chapter.) Although there is evidence that palms may be dispersal limited to some degree (Kissling et al. 2012), the dispersal of palms across long distances is accepted to have been enhanced by frugivores, especially birds (Zona and Henderson 1989) and, less frequently, by their floating seeds (Baker and Couvreur 2012). 
Understanding the diversity, distribution, and dispersal of palms is integral to supporting their conservation (Dransfield et al. 2008, Eiserhardt et al. 2011, Bacon et al. 2012). Given the limited availability of resources available for research and conservation efforts in Maluku, and the relative ease with which palms can be identified (if they are observed while fertile) in comparison to other tropical trees, palms are excellent candidates for utilizing in a variety of conservation and monitoring efforts. For example, palms that were found in the NP to be restricted to primary forest habitats (e.g., Drymophloeus spp. and Hydriastele spp.) may be indicative of good habitat quality in park-wide monitoring studies. While further research would be necessary to refine such qualitative rankings of palms that could be used in habitat quality monitoring studies, given the shortage of resources in Halmahera there is nonetheless value in this (albeit imperfect) approach. Furthermore, Moluccan palms not found in the NP could be prioritized for ex-situ germplasm collections in the national botanic gardens of Indonesia.

Palms that are found only in perennially wet habitats in the NP (e.g., Nypa fruticans) could be monitored over time and their distributions examined in order to learn about plant and habitat responses to extreme weather events (i.e., extreme droughts or floods). Studying the dispersal of large-fruited palms is another relevant research direction, in that it may shed light upon the dispersal of other large-fruited tropical trees and impart insights regarding dispersal redundancy or obligate dispersers for other trees of Maluku. Some palms (e.g., Pigafetta filaris) exhibit relatively rapid growth in open areas, and could have value in restoration efforts; while restoration is a highly complex endeavor, 
working to not only restore plant species but also to restore plant-animal interactions

(e.g., seed dispersal mutualisms) is increasingly important (Kaiser-Bunbury et al. 2010,

Howe 2016).

\section{REFERENCES}

Audley-Charles, M. G. (1993). Geological evidence bearing upon the Pliocene emergence of Seram, an island colo- nizable by land plants and animals, in Natural History of Seram, Eds. I. D. Edwards, A. A. Macdonald, and J. Proctor. Intercept Ltd, Andover.

Bacon, C. D., Baker, W. J., \& Simmons, M. P. (2012). Miocene dispersal drives island radiations in the palm tribe Trachycarpeae (Arecaceae). Systematic Biology, 61 (3), 426-442.

Baker, W.P. (2015). A revised delimitation of the rattan genus Calamus (Arecaceae). Phytotaxa 197 (2): 139-152.

Baker, W.P. and Couvreur, T.L.P. (2012). Biogeography and distribution patterns of Southeast Asian palms, in Biotic Evolution and Environmental Change in Southeast Asia, Eds. D. J. Gower et al. The Systematics Association, Cambridge University Press.

Baker, W.P. and Couvreur, T.L.P. (2013a). Global biogeography and diversification of palms sheds light on the evolution of tropical lineages. I. Historical biogeography. Journal of Biogeography 40: 274-285.

Baker, W.P. and Couvreur, T.L.P. (2013b). Global biogeography and diversification of palms sheds light on the evolution of tropical lineages. II. Diversification history and origin of regional assemblages. Journal of Biogeography 40: 286-298.

Baker, W.P. and Dransfield, P. (2014). New rattans from New Guinea (Calamus, Arecaceae). Phytotaxa 163 (4): 181-215.

Baker, W.P. and Loo, A.H.B. (2004). A synopsis of the genus Hydriastele (Arecaceae). Kew Bulletin 59: 61-68.

Banka, R., and Baker, W.J. (2009). A monograph of the genus Rhopaloblaste (Arecaceae). Kew Bulletin 59: 47-60. 
Barfod, A.S., and Heatubun, C.D. (2009). Two new species of Licuala Thunb. (Arecaceae: Coryphoideae) from North Moluccas and Western New Guinea. Kew Bulletin 64: 553-557.

de Jong, R. (1998). Halmahera and Seram: different histories, but similar butterfly faunas, in Biogeography and Geological Evolution of SE Asia, Eds. R. Hall and J. D. Holloway, Backhuys Publishers, Leiden, The Netherlands

Dransfield, J. (1987). Bicentric distributions in Malesia as exemplified by palms. In Biogeographical Evolution of the Malay Archipelago, ed. T. C. Whitmore. Oxford: Clarendon Press.

Dransfield, J., Uhl, N.W., Asmussen, C.B., Baker, W.J., Harley, M.M. \& Lewis, C.E. (2008). Genera Palmarum - the evolution and classification of palms, 2nd edn. Royal Botanic Gardens, Kew, Richmond, UK.

Eiserhardt, W. L., Svenning, J. C., Kissling, W. D., \& Balslev, H. (2011). Geographical ecology of the palms (Arecaceae): determinants of diversity and distributions across spatial scales. Annals of Botany, 108 (8), 1391-1416.

Gardiner, L. M., Dransfield, J., Marcus, J., and Baker, W.J. (2012). Heterospathe barfodii, a new species from Papua New Guinea. Palms 56 (2): 91-100.

Govaerts, R., Dransfield, J., Zona, S.F, Hodel, D.R. \& Henderson, A. (2016). World Checklist of Arecaceae. Facilitated by the Royal Botanic Gardens, Kew. Published on the Internet; http://apps.kew.org/wcsp/ Retrieved 2016-11-24

Hahn, W. J. and Sytsma, K. J. (1999). Molecular systematics and biogeography of the Southeast Asian genus Caryota (Palmae). Systematic Botany, 24, 558-80.

Hall, R. (1987). Plate boundary evolution in the Halmahera region, Indonesia Tectonophysics 144: 337-352

Heatubun, C. D., Dransfield, J., Flynn, T., Tjitrosoedirdjo, S. S., Mogea, J. P., \& Baker, W. J. (2012). A monograph of the betel nut palms (Areca: Arecaceae) of East Malesia. Botanical Journal of the Linnean Society, 168(2): 147-173.

Heatubun, C.D., Zona, S., and Baker, W.J. (2014). Three new genera of arecoid palm (Arecaceae) from eastern Malesia. Kew Bulletin 69: 9525.

Howe, H. F. (2016). Making dispersal syndromes and networks useful in tropical conservation and restoration. Global Ecology and Conservation 6: 152-178. 
Kaiser-Bunbury, C.N., Traveset, A., and Hansen, D.M. (2010). Conservation and restoration of plant-animal mutualisms on oceanic islands. Perspectives in Plant Ecology, Evolution and Systematics 12: 131-143.

Kissling, W.D., Eiserhardt, W.L., Baker, W.J., Borchsenius, F., Couvreur, T.L.P., Balslev, H. and Svenning, J.C. (2012). Cenozoic imprints on the phylogenetic structure of palm species assemblages worldwide. Proceedings of the National Academy of Sciences USA, 109: 7379-7384.

Monk, K. A., de Fretes, Y., and Reksodiharjo-Lilley, G. (1997). The Ecology of Nusa Tenggara and Maluku. Periplus Edition, Singapore

Myers, N., Mittermeier, R. A., Mittermeier, C. G., Da Fonseca, G. A., \& Kent, J. (2000). Biodiversity hotspots for conservation priorities. Nature, 403 (6772), 853-858.

Powling, A. (2009). The palms of Buton, Indonesia, an island in Wallacea. Palms 53 (2) 84-91.

Rumphius, G.E. (1741) Herbarium Amboinense, Netherlands.

Rumphius, G.E. (2011). The Ambonese Herbal, translated by E.M. Beekman. Yale University Press and National Tropical Botanic Garden, New Haven and London.

Suparan, P., R.A.C. Dam, S. van der Kaars, \& T.E. Wong. (2001). Late Quaternary tropical lowland environments on Halmahera, Indonesia. Palaeogeography, Palaeoclimatology, Palaeoecology 171: 229-258

Vane-Wright, R. I. and Peggie, D. (1994). The butterflies of Northern and Central Maluku: diversity, endemism, biogeography, and conservation priorities. Tropical Biodiversity 2: 212-230.

Wallace, A. R. (1860). On the zoological geography of the Malay Archipelago. Journal of the Proceedings of the Linnean Society, Zoology, 4: 172-84.

Zona, S., and Henderson, A. (1989). A review of animal-mediated seed dispersal of palms. Selbyana 11: 6-21. 


\section{FIGURES AND TABLES}

Figure 1. Map of Halmahera island highlighting palm study sites, inset within the Wallacea biodiversity hotspot of Indonesia.

Table 1. List of the palms (Arecaceae) of Maluku, Indonesia, noting regional endemism.

Table 2. Summary of the taxonomic diversity of the palms (Arecaceae) of Maluku, Indonesia.

Table 3. Annotated list of the palms of Aketajawe-Lolobata National Park, Halmahera, Indonesia.

Table 4. Distribution patterns of palm genera found in Maluku, Indonesia. 
Figure 1. Map of Halmahera island highlighting palm study areas, inset within the Wallacea biodiversity hotspot of Indonesia.

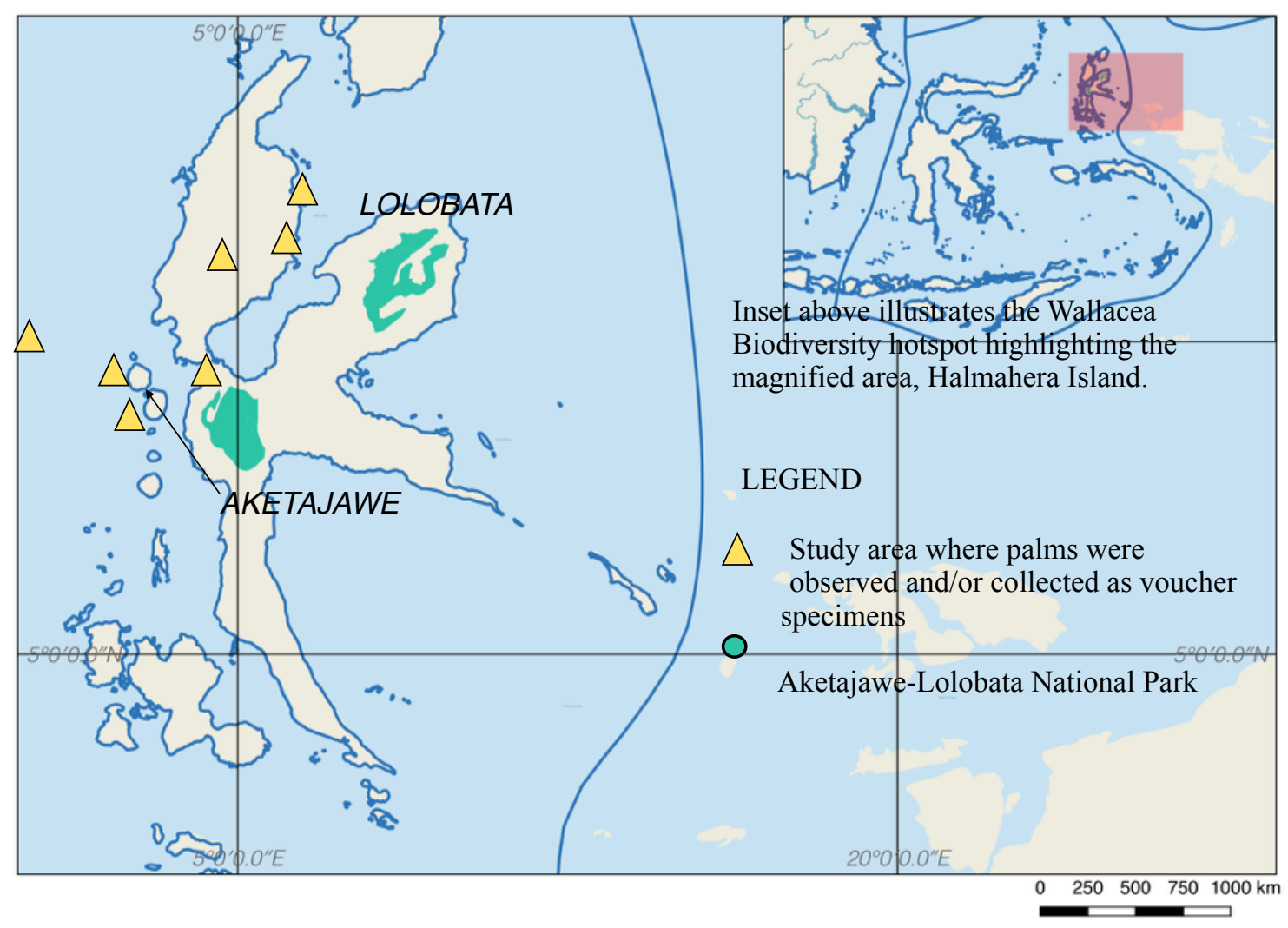


Table 1. List of the palms (Arecaceae) of Maluku, Indonesia, noting regional endemism. In "Endemic to Maluku" column: $\mathrm{N}=$ not endemic, $\mathrm{Y}=$ species is endemic.

\begin{tabular}{|c|c|c|c|}
\hline Subfamily & Tribe & Species & $\begin{array}{l}\text { Endemic } \\
\text { to } \\
\text { Maluku }\end{array}$ \\
\hline Arecoideae & Areceae & Areca vestiaria Giseke & $\mathrm{N}$ \\
\hline Arecoideae & Areceae & Areca catechu L. & $\mathrm{N}$ \\
\hline Arecoideae & Areceae & Areca macrocalyx Zipp. ex Blume & $\mathrm{N}$ \\
\hline Arecoideae & Areceae & Calyptrocalyx spicatus (Lam.) Blume & $\mathrm{Y}$ \\
\hline Arecoideae & Areceae & $\begin{array}{l}\text { Drymophloeus oliviformis (Giseke) } \\
\text { Mart. }\end{array}$ & $\mathrm{N}$ \\
\hline Arecoideae & Areceae & $\begin{array}{l}\text { Drymophloeus litigiosus (Becc.) } \\
\text { H.E.Moore }\end{array}$ & $\mathrm{N}$ \\
\hline Arecoideae & Areceae & $\begin{array}{l}\text { Heterospathe glauca (Scheff.) } \\
\text { H.E.Moore }\end{array}$ & $\mathrm{Y}$ \\
\hline Arecoideae & Areceae & Heterospathe elata Scheff. var. elata & $\mathrm{N}$ \\
\hline Arecoideae & Areceae & $\begin{array}{l}\text { Hydriastele beguinii (Burret) } \\
\text { W.J.Baker \& Loo }\end{array}$ & $\mathrm{Y}$ \\
\hline Arecoideae & Areceae & $\begin{array}{l}\text { Hydriastele oxypetala (Burret) } \\
\text { W.J.Baker \& Loo }\end{array}$ & $\mathrm{Y}$ \\
\hline Arecoideae & Areceae & $\begin{array}{l}\text { Hydriastele moluccana (Becc.) } \\
\text { W.J.Baker \& Loo }\end{array}$ & $\mathrm{Y}$ \\
\hline Arecoideae & Areceae & $\begin{array}{l}\text { Hydriastele microcarpa (Scheff.) } \\
\text { W.J.Baker \& Loo }\end{array}$ & $\mathrm{Y}$ \\
\hline Arecoideae & Areceae & $\begin{array}{l}\text { Jailoloa halmaherensis (Heatubun) } \\
\text { Heatubun \& WJ Baker }\end{array}$ & $\mathrm{Y}$ \\
\hline Arecoideae & Areceae & Pinanga globulifera (Lam.) Blume & $\mathrm{Y}$ \\
\hline Arecoideae & Areceae & $\begin{array}{l}\text { Pinanga rumphiana (Mart.) J.Dransf. } \\
\text { \& Govaerts }\end{array}$ & $\mathrm{N}$ \\
\hline Arecoideae & Areceae & $\begin{array}{l}\text { Ptychosperma propinquum (Becc.) } \\
\text { Becc. ex Martelli }\end{array}$ & $\mathrm{N}$ \\
\hline Arecoideae & Areceae & Rhopaloblaste ceramica (Miq.) Burret & $\mathrm{N}$ \\
\hline Arecoideae & Oranieae & Orania palindan (Blanco) Merr. & $\mathrm{N}$ \\
\hline
\end{tabular}




\begin{tabular}{|c|c|c|c|}
\hline Subfamily & Tribe & Species & $\begin{array}{l}\text { Endemic } \\
\text { to } \\
\text { Maluku }\end{array}$ \\
\hline Calamoideae & Calameae & Calamus albus Pers & $\mathrm{Y}$ \\
\hline Calamoideae & Calameae & Calamus aruensis Becc & $\mathrm{N}$ \\
\hline Calamoideae & Calameae & Calamus beguinii (Burret) W.J.Baker & $\mathrm{Y}$ \\
\hline Calamoideae & Calameae & Calamus buroensis Mart. ex Walp. & $\mathrm{Y}$ \\
\hline Calamoideae & Calameae & Calamus calapparius Mart. & $\mathrm{Y}$ \\
\hline Calamoideae & Calameae & Calamus cawa Blume & $\mathrm{Y}$ \\
\hline Calamoideae & Calameae & Calamus equestris Willd & $\mathrm{Y}$ \\
\hline Calamoideae & Calameae & Calamus graminosus Blume & $\mathrm{Y}$ \\
\hline Calamoideae & Calameae & Calamus halmaherensis Burret & $\mathrm{Y}$ \\
\hline Calamoideae & Calameae & $\begin{array}{l}\text { Calamus heteracanthus Zipp. ex } \\
\text { Blume }\end{array}$ & $\mathrm{N}$ \\
\hline Calamoideae & Calameae & Calamus niger Willd. & $\mathrm{Y}$ \\
\hline Calamoideae & Calameae & Calamus pisicarpus Blume & $\mathrm{Y}$ \\
\hline Calamoideae & Calameae & Calamus robinsonianus Becc & $\mathrm{Y}$ \\
\hline Calamoideae & Calameae & Calamus rumphii Blume & $\mathrm{Y}$ \\
\hline Calamoideae & Calameae & Calamus validus W.J.Baker & $\mathrm{Y}$ \\
\hline Calamoideae & Calameae & Korthalsia celebica Becc. & $\mathrm{N}$ \\
\hline Calamoideae & Calameae & Metroxylon sagu Rottb. & $\mathrm{N}$ \\
\hline Calamoideae & Calameae & Pigafetta filaris (Giseke) Becc. & $\mathrm{N}$ \\
\hline Calamoideae & Calameae & Salacca zalacca (Gaertn.) Voss & $\mathrm{N}$ \\
\hline Coryphoideae & Caryoteae & Arenga microcarpa Becc. & $\mathrm{N}$ \\
\hline Coryphoideae & Caryoteae & Arenga pinnata (Wurmb) Merr. & $\mathrm{N}$ \\
\hline Coryphoideae & Caryoteae & Caryota rumphiana Mart & $\mathrm{N}$ \\
\hline Coryphoideae & Corypheae & Corypha utan Lam. & $\mathrm{N}$ \\
\hline Coryphoideae & Trachycarpeae & $\begin{array}{l}\text { Licuala adscendens Barfod \& } \\
\text { Heatubun }\end{array}$ & $\mathrm{Y}$ \\
\hline Coryphoideae & Trachycarpeae & Licuala flexuosa Burret & $\mathrm{Y}$ \\
\hline
\end{tabular}




\begin{tabular}{|l|l|l|l|}
\hline Subfamily & Tribe & Species & $\begin{array}{l}\text { Endemic } \\
\text { to } \\
\text { Maluku }\end{array}$ \\
\hline Coryphoideae & Trachycarpeae & Licuala rumphii Blume & Y \\
\hline Coryphoideae & Trachycarpeae & Pholidocarpus ihur (Giseke) Blume & N \\
\hline Coryphoideae & Trachycarpeae & Saribus rotundifolius (Lam.) Blume & N \\
\hline Nypoideae & N/A & Nypa fruticans Wurmb & N \\
\hline
\end{tabular}


Table 2. Summary of the taxonomic diversity of the palms (Arecaceae) of Maluku, Indonesia.

\begin{tabular}{|l|l|l|l|l|}
\hline Subfamily & Tribe & $\begin{array}{l}\text { Number of } \\
\text { genera in } \\
\text { tribe in } \\
\text { Maluku }\end{array}$ & $\begin{array}{l}\text { Number of } \\
\text { species in } \\
\text { Maluku }\end{array}$ & $\begin{array}{l}\text { Endemic } \\
\text { species in } \\
\text { Maluku }\end{array}$ \\
\hline Arecoideae & Areceae & 9 & 17 & 8 \\
\hline & Oranieae & 1 & 1 & 0 \\
\hline Calamoideae & Calameae & 5 & 19 & 11 \\
\hline Coryphoideae & Caryoteae & 2 & 3 & 0 \\
\hline & Corypheae & 1 & 1 & 0 \\
\hline & Trachycarpeae & 3 & 5 & 3 \\
\hline Nypoideae & N/A & 1 & 1 & 0 \\
\hline
\end{tabular}


Table 3. Annotated list of the palms of Aketajawe-Lolobata National Park, Halmahera, Indonesia.

In "Presence in Aketajawe-Lolobata NP" column: $A=$ present in Aketajawe block, $\mathrm{L}=$ present in Lolobata block, and A, L=present in both NP blocks; (s) = a voucher specimen was collected, (o) = an observation of presence was made. In "Endemic to Maluku" column: $\mathrm{N}=$ not endemic, $\mathrm{Y}=$ species is endemic, and $\mathrm{U}=$ unknown status.

\begin{tabular}{|c|c|c|c|c|}
\hline Species & $\begin{array}{l}\text { Presence in } \\
\text { Aketajawe- } \\
\text { Lolobata } \\
\text { NP }\end{array}$ & $\begin{array}{l}\text { Habitat } \\
\text { Occurrences }\end{array}$ & $\begin{array}{l}\text { Estimated } \\
\text { frequency of } \\
\text { occurrence } \\
\text { within observed } \\
\text { habitats }\end{array}$ & $\begin{array}{l}\text { Endemic } \\
\text { to } \\
\text { Maluku }\end{array}$ \\
\hline $\begin{array}{l}\text { Areca vestiaria } \\
\text { Giseke }\end{array}$ & $\begin{array}{l}\text { A, L } \\
\text { (s) }\end{array}$ & $\begin{array}{l}\text { Primary forest } \\
\text { especially along } \\
\text { rivers; karst } \\
\text { forest; disturbed } \\
\text { primary forest; } \\
\text { elev. }<500 \mathrm{~m}\end{array}$ & Common & $\mathrm{N}$ \\
\hline Areca catechu L. & $\begin{array}{l}\text { A } \\
\text { (o) }\end{array}$ & $\begin{array}{l}\text { Secondary forest } \\
\text { (probably from } \\
\text { cultivation) }\end{array}$ & $\begin{array}{l}\text { Rare (probably } \\
\text { cultivated) }\end{array}$ & $\mathrm{N}$ \\
\hline $\begin{array}{l}\text { Calyptrocalyx } \\
\text { spicatus (Lam.) } \\
\text { Blume }\end{array}$ & $\begin{array}{l}\mathrm{L} \\
(\mathrm{s})\end{array}$ & $\begin{array}{l}\text { Primary forest; } \\
\text { karst forest; } \\
\text { disturbed primary } \\
\text { forest; } \\
\text { premontane } \\
\text { forest; elev. } \\
>250 \mathrm{~m}\end{array}$ & Rare & $\mathrm{Y}$ \\
\hline $\begin{array}{l}\text { Drymophloeus } \\
\text { oliviformis } \\
\text { (Giseke) Mart. }\end{array}$ & $\begin{array}{l}\text { A, L } \\
\text { (s) }\end{array}$ & $\begin{array}{l}\text { Primary forest; } \\
\text { dense karst forest; } \\
\text { elev. }<700 \mathrm{~m}\end{array}$ & Occasional & $\mathrm{N}$ \\
\hline $\begin{array}{l}\text { Drymophloeus } \\
\text { litigiosus (Becc.) } \\
\text { H.E.Moore }\end{array}$ & $\begin{array}{l}\text { A, L } \\
\text { (s) }\end{array}$ & $\begin{array}{l}\text { Primary forest; } \\
\text { dense karst forest; } \\
\text { premontane forest } \\
\text { elev. }<800 \mathrm{~m}\end{array}$ & $\begin{array}{l}\text { Common in small } \\
\text { areas of primary } \\
\text { forest; occasional in } \\
\text { most occurrences }\end{array}$ & $\mathrm{N}$ \\
\hline $\begin{array}{l}\text { Heterospathe } \\
\text { glauca cf. } \\
\text { (Scheff.) } \\
\text { H.E.Moore }\end{array}$ & $\begin{array}{l}\mathrm{L} \\
(\mathrm{s})\end{array}$ & $\begin{array}{l}\text { Primary forest; } \\
\text { elev. }>230 \mathrm{~m} \text {, } \\
<500 \mathrm{~m}\end{array}$ & Rare & $\mathrm{Y}$ \\
\hline $\begin{array}{l}\text { Hydriastele } \\
\text { beguinii (Burret) } \\
\text { W.J.Baker \& Loo }\end{array}$ & $\begin{array}{l}\text { A, L } \\
\text { (s) }\end{array}$ & $\begin{array}{l}\text { Primary forest; } \\
\text { karst forest with } \\
\text { sparse substrate; } \\
\text { dense karst forest; } \\
\text { elev. }<500 \mathrm{~m}\end{array}$ & Occasional & $\mathrm{Y}$ \\
\hline
\end{tabular}




\begin{tabular}{|c|c|c|c|c|}
\hline Species & $\begin{array}{l}\text { Presence in } \\
\text { Aketajawe- } \\
\text { Lolobata } \\
\text { NP }\end{array}$ & $\begin{array}{l}\text { Habitat } \\
\text { Occurrences }\end{array}$ & $\begin{array}{l}\text { Estimated } \\
\text { frequency of } \\
\text { occurrence } \\
\text { within observed } \\
\text { habitats }\end{array}$ & $\begin{array}{l}\text { Endemic } \\
\text { to } \\
\text { Maluku }\end{array}$ \\
\hline $\begin{array}{l}\text { Hydriastele } \\
\text { microcarpa } \\
\text { (Scheff.) } \\
\text { W.J.Baker \& Loo }\end{array}$ & $\begin{array}{l}\text { A, L } \\
\text { (s) }\end{array}$ & $\begin{array}{l}\text { Primary forest; } \\
\text { karst forest with } \\
\text { sparse substrate; } \\
\text { dense karst forest; } \\
\text { elev. }<500 \mathrm{~m}\end{array}$ & Occasional & $\mathrm{Y}$ \\
\hline $\begin{array}{l}\text { Pinanga } \\
\text { rumphiana (Mart.) } \\
\text { J.Dransf. \& } \\
\text { Govaerts }\end{array}$ & $\begin{array}{l}\text { A, L } \\
\text { (s) }\end{array}$ & $\begin{array}{l}\text { Primary forest; } \\
\text { karst forest; } \\
\text { disturbed primary } \\
\text { forest; rarely } \\
\text { secondary forest; } \\
\text { elev. }<600 \mathrm{~m}\end{array}$ & $\begin{array}{l}\text { Common in patches } \\
\text { especially along } \\
\text { rivers; otherwise } \\
\text { occasional }\end{array}$ & $\mathrm{N}$ \\
\hline $\begin{array}{l}\text { Ptychosperma } \\
\text { propinquum } \\
\text { (Becc.) Becc. ex } \\
\text { Martelli }\end{array}$ & $\begin{array}{l}\text { A } \\
(\mathrm{s})\end{array}$ & $\begin{array}{l}\text { Primary forest; } \\
\text { elev. }<400 \mathrm{~m}\end{array}$ & Occasional & $\mathrm{N}$ \\
\hline $\begin{array}{l}\text { Rhopaloblaste } \\
\text { ceramica (Miq.) } \\
\text { Burret }\end{array}$ & $\begin{array}{l}\text { A, L } \\
\text { (o) }\end{array}$ & $\begin{array}{l}\text { Primary forest; } \\
\text { karst forest; open } \\
\text { alluvial habitats; } \\
\text { disturbed primary } \\
\text { forest; elev. } \\
<500 \mathrm{~m}\end{array}$ & Occasional & $\mathrm{N}$ \\
\hline $\begin{array}{l}\text { Orania palindan } \\
\text { (Blanco) Merr. }\end{array}$ & $\begin{array}{l}\text { A, L } \\
\text { (s) }\end{array}$ & $\begin{array}{l}\text { Primary forest; } \\
\text { karst forest; } \\
\text { alluvial habitats; } \\
\text { secondary forest; } \\
\text { semi-open } \\
\text { agroforest; elev. } \\
<500 \mathrm{~m}\end{array}$ & $\begin{array}{l}\text { Common in some } \\
\text { patches of alluvial } \\
\text { habitat; otherwise } \\
\text { occasional }\end{array}$ & $\mathrm{N}$ \\
\hline $\begin{array}{l}\text { Calamus albus } \\
\text { Pers }\end{array}$ & $\begin{array}{l}\text { A, L } \\
(\mathrm{s})\end{array}$ & $\begin{array}{l}\text { Primary forest; } \\
\text { karst forest; } \\
\text { alluvial habitats; } \\
\text { elev. }<700 \mathrm{~m}\end{array}$ & $\begin{array}{l}\text { Common in several } \\
\text { areas; occasional } \\
\text { otherwise }\end{array}$ & $\mathrm{Y}$ \\
\hline $\begin{array}{l}\text { Calamus } \\
\text { calapparius Mart. }\end{array}$ & $\begin{array}{l}\text { A, L } \\
\text { (s) }\end{array}$ & $\begin{array}{l}\text { Primary forest; } \\
\text { karst forests; elev. } \\
<700 \mathrm{~m}\end{array}$ & Occasional & $\mathrm{Y}$ \\
\hline $\begin{array}{l}\text { Calamus } \\
\text { heteracanthus } \\
\text { Zipp. ex Blume }\end{array}$ & $\begin{array}{l}\text { A } \\
\text { (o) }\end{array}$ & $\begin{array}{l}\text { Primary forest; } \\
\text { karst forests; elev. } \\
>150 \mathrm{~m},<700 \mathrm{~m}\end{array}$ & Occasional & $\mathrm{N}$ \\
\hline
\end{tabular}




\begin{tabular}{|c|c|c|c|c|}
\hline Species & $\begin{array}{l}\text { Presence in } \\
\text { Aketajawe- } \\
\text { Lolobata } \\
\text { NP }\end{array}$ & $\begin{array}{l}\text { Habitat } \\
\text { Occurrences }\end{array}$ & $\begin{array}{l}\text { Estimated } \\
\text { frequency of } \\
\text { occurrence } \\
\text { within observed } \\
\text { habitats }\end{array}$ & $\begin{array}{l}\text { Endemic } \\
\text { to } \\
\text { Maluku }\end{array}$ \\
\hline Calamus sp. A & $\begin{array}{l}\text { A, L } \\
\text { (o) }\end{array}$ & $\begin{array}{l}\text { Primary forest } \\
\text { especially along } \\
\text { rivers; elev. } \\
<350 \mathrm{~m} \text {. }\end{array}$ & Occasional & $\mathrm{U}$ \\
\hline Calamus sp. B & $\begin{array}{l}\mathrm{L} \\
\text { (o) }\end{array}$ & $\begin{array}{l}\text { Primary forest, } \\
\text { hilly karst forest } \\
\text { with thin soils/ } \\
\text { exposed substrate } \\
\text { in places; elev. } \\
>150,<700 \mathrm{~m} \text {. }\end{array}$ & Occasional & $\mathrm{U}$ \\
\hline Calamus sp. C & $\begin{array}{l}\mathrm{L} \\
(\mathrm{o})\end{array}$ & $\begin{array}{l}\text { Primary forest, } \\
\text { karst forest, } \\
<700 \mathrm{~m}\end{array}$ & $\begin{array}{l}\text { Occasional, except } \\
\text { common in several } \\
\text { areas (steep } \\
\text { hillsides) forming } \\
\text { dense stands }\end{array}$ & $\mathrm{U}$ \\
\hline $\begin{array}{l}\text { Pigafetta filaris } \\
\text { (Giseke) Becc. }\end{array}$ & $\begin{array}{l}\text { A, L } \\
\text { (o) }\end{array}$ & $\begin{array}{l}\text { Primary forest; } \\
\text { karst forests; } \\
\text { disturbed forests; } \\
\text { secondary forests; } \\
\text { open areas } \\
\text { adjacent to rivers, } \\
\text { elev. }<600 \mathrm{~m}\end{array}$ & $\begin{array}{l}\text { Occasional; rarely } \\
\text { becoming common } \\
\text { in some disturbed } \\
\text { habitats }\end{array}$ & $\mathrm{N}$ \\
\hline $\begin{array}{l}\text { Metroxylon sagu } \\
\text { (Giseke) Becc. }\end{array}$ & $\begin{array}{l}\mathrm{L} \\
\text { (o) }\end{array}$ & $\begin{array}{l}\text { Perennially wet } \\
\text { forest; sagu } \\
\text { swamps }\end{array}$ & $\begin{array}{l}\text { Common only in } \\
\text { this habitat type }\end{array}$ & $\mathrm{N}$ \\
\hline $\begin{array}{l}\text { Arenga pinnata } \\
\text { (Wurmb) Merr. }\end{array}$ & $\begin{array}{l}\text { A } \\
\text { (o) }\end{array}$ & $\begin{array}{l}\text { Secondary forest } \\
\text { (possibly from } \\
\text { cultivation) }\end{array}$ & $\begin{array}{l}\text { Rare (probably } \\
\text { cultivated) }\end{array}$ & $\mathrm{N}$ \\
\hline $\begin{array}{l}\text { Caryota } \\
\text { rumphiana Mart }\end{array}$ & $\begin{array}{l}\text { A, L } \\
\text { (o) }\end{array}$ & $\begin{array}{l}\text { Secondary forest; } \\
\text { disturbed primary } \\
\text { forest }\end{array}$ & Occasional & $\mathrm{N}$ \\
\hline $\begin{array}{l}\text { Corypha utan } \\
\text { Lam. }\end{array}$ & $\begin{array}{l}\text { A } \\
\text { (o) }\end{array}$ & $\begin{array}{l}\text { Disturbed primary } \\
\text { forest; primary } \\
\text { forest }\end{array}$ & Rare & $\mathrm{N}$ \\
\hline
\end{tabular}




\begin{tabular}{|c|c|c|c|c|}
\hline Species & $\begin{array}{l}\text { Presence in } \\
\text { Aketajawe- } \\
\text { Lolobata } \\
\text { NP }\end{array}$ & $\begin{array}{l}\text { Habitat } \\
\text { Occurrences }\end{array}$ & $\begin{array}{l}\text { Estimated } \\
\text { frequency of } \\
\text { occurrence } \\
\text { within observed } \\
\text { habitats }\end{array}$ & $\begin{array}{l}\text { Endemic } \\
\text { to } \\
\text { Maluku }\end{array}$ \\
\hline $\begin{array}{l}\text { Licuala flexuosa } \\
\text { Burret }\end{array}$ & $\begin{array}{l}\text { A } \\
\text { (o) }\end{array}$ & $\begin{array}{l}\text { Primary forest; } \\
\text { minimally } \\
\text { disturbed primary } \\
\text { forest; on thins } \\
\text { soils upon } \\
\text { limestone } \\
\text { adjacent to rivers; } \\
\text { elev. }<500 \mathrm{~m}\end{array}$ & Occasional & $\mathrm{Y}$ \\
\hline $\begin{array}{l}\text { Licuala rumphii } \\
\text { Blume }\end{array}$ & $\begin{array}{l}\text { A, L } \\
\text { (s) }\end{array}$ & $\begin{array}{l}\text { Primary forest; } \\
\text { disturbed primary } \\
\text { forest; secondary } \\
\text { forest; elev. } \\
<700 \mathrm{~m}\end{array}$ & $\begin{array}{l}\text { Occasional in } \\
\text { primary forest } \\
\text { habitats; rare in } \\
\text { secondary forest }\end{array}$ & $\mathrm{Y}$ \\
\hline $\begin{array}{l}\text { Saribus } \\
\text { rotundifolius } \\
\text { (Lam.) Blume }\end{array}$ & $\begin{array}{l}\text { A, L } \\
\text { (o) }\end{array}$ & $\begin{array}{l}\text { Primary forest; } \\
\text { karst forest; } \\
\text { disturbed primary } \\
\text { forest; secondary } \\
\text { forest; elev. } \\
>200 \mathrm{~m},<700 \mathrm{~m}\end{array}$ & Occasional & $\mathrm{N}$ \\
\hline $\begin{array}{l}\text { Nypa fruticans } \\
\text { Wurmb }\end{array}$ & $\begin{array}{l}\mathrm{L} \\
\text { (o) }\end{array}$ & $\begin{array}{l}\text { Swampy, } \\
\text { perennially wet } \\
\text { habitat with Nypa } \\
\text { dominant }\end{array}$ & $\begin{array}{l}\text { Common only in } \\
\text { this habitat type }\end{array}$ & $\mathrm{N}$ \\
\hline
\end{tabular}


Table 4. Distribution patterns of palm genera found in Maluku, Indonesia.

In "Notes on the distribution..." column: $\mathrm{B}=$ bimodal distribution, with species occurring both East and West of Wallace's Line ("WL"), $\mathrm{C}=\mathrm{a}$ widespread or cosmopolitan distribution in tropical areas, $\mathrm{E}=$ species occurring primarily East of WL, W= species occurring primarily West of WL.

\begin{tabular}{|c|c|c|}
\hline Genus & $\begin{array}{l}\text { Number of } \\
\text { Moluccan } \\
\text { species in genus }\end{array}$ & $\begin{array}{l}\text { Notes on the distribution of the } \\
\text { genus and centers of diversity in } \\
\text { Malesia }\end{array}$ \\
\hline Calamus & 15 & $\begin{array}{l}\text { (B) Three centers of diversity in Malesia: } \\
\text { West of Wallace's Line ("WL"), especially } \\
\text { Borneo and the Philippines. Also occurring } \\
\text { East of WL (especially Papua), but } \\
\text { depauperate in Wallacea in comparison } \\
\text { with other areas (although it is the most } \\
\text { speciose genus of palms in Maluku). }\end{array}$ \\
\hline Hydriastele & 4 & $\begin{array}{l}\text { (E) Center of diversity is in Papua and the } \\
\text { genus ranges into the W. Pacific. The genus } \\
\text { also speciated in Wallacea, although } \\
\text { Wallacean diversity is far lower than that of } \\
\text { Papua }\end{array}$ \\
\hline Areca & 3 & $\begin{array}{l}\text { (W) Distributed primarily West of WL } \\
\text { with Borneo the center of diversity; species } \\
\text { also occur E of WL extending to the } \\
\text { Solomon islands. }\end{array}$ \\
\hline Licuala & 3 & $\begin{array}{l}\text { (B) Very high diversity into the far West of } \\
\text { WL (especially Borneo) as well as East of } \\
\text { WL (in Papua), but depauperate in } \\
\text { Wallacea. }\end{array}$ \\
\hline Arenga & 2 & $\begin{array}{l}\text { (W) Most of the species diversity occurs } \\
\text { West of WL. }\end{array}$ \\
\hline Drymophloeus & 2 & $\begin{array}{l}\text { (E) Species in the genus occur from } \\
\text { Wallacea, through Papua, into the W. } \\
\text { Pacific. }\end{array}$ \\
\hline Heterospathe & 2 & $\begin{array}{l}\text { (B) Absent from far West of WL, except in } \\
\text { the Philippines where there is high } \\
\text { diversity. The genus is depauperate in } \\
\text { Wallacea but diverse in Papua. }\end{array}$ \\
\hline Pinanga & 2 & $\begin{array}{l}\text { (W) Primarily distributed West of WL, } \\
\text { although the genus crosses into Wallacea } \\
\text { and Papua }\end{array}$ \\
\hline Calyptrocalyx & 1 & $\begin{array}{l}\text { (E) Nearly all species diversity of the } \\
\text { genus occurs in Papua }\end{array}$ \\
\hline
\end{tabular}




\begin{tabular}{|c|c|c|}
\hline Genus & $\begin{array}{l}\text { Number of } \\
\text { Moluccan } \\
\text { species in genus }\end{array}$ & $\begin{array}{l}\text { Notes on the distribution of the } \\
\text { genus and centers of diversity in } \\
\text { Malesia }\end{array}$ \\
\hline Caryota & 1 & $\begin{array}{l}\text { (W) Probably dispersed from West to East, } \\
\text { with most species West of WL; however, } \\
\text { the Philippines has } 3 \text { species compared } \\
\text { with most other areas which have only } 1 \text { or } \\
2\end{array}$ \\
\hline Corypha & 1 & $\begin{array}{l}\text { (C) Only one taxon, C. utan, in Malesia } \\
\text { except for the Philippines which has an } \\
\text { additional endemic taxon. Diversity of the } \\
\text { genus is higher outside of Malesia, which } \\
\text { could be because of relatively recent } \\
\text { dispersal into the region. }\end{array}$ \\
\hline Jailoloa & 1 & $\begin{array}{l}\text { (E) Monotypic genus endemic to } \\
\text { Halmahera island }\end{array}$ \\
\hline Korthalsia & 1 & $\begin{array}{l}\text { (W) Primarily distributed West of WL, } \\
\text { although the genus crosses into Wallacea } \\
\text { and Papua }\end{array}$ \\
\hline Metroxylon & 1 & $\begin{array}{l}\text { (E) Only found East of WL where one } \\
\text { taxon is widespread, and other taxa extend } \\
\text { into the W. Pacific }\end{array}$ \\
\hline Nypa & 1 & $\begin{array}{l}\text { (C) Only one taxon in the genus, Nypa } \\
\text { fruticans, which is widespread in humid } \\
\text { tropical environs }\end{array}$ \\
\hline Orania & 1 & $\begin{array}{l}\text { (B) Main center of diversity is in Papua, } \\
\text { with far fewer species in the Philippines } \\
\text { and one extending into W. Malesia and } \\
\text { Thailand. A disjunct group of } 3 \text { taxa is in } \\
\text { Madagascar. }\end{array}$ \\
\hline Pholidocarpus & 1 & $\begin{array}{l}\text { (W) Primarily distributed West of WL, } \\
\text { although the genus extends into Wallacea. }\end{array}$ \\
\hline Pigafetta & 1 & $\begin{array}{l}\text { (E) No species occur West of WL; the } \\
\text { genus ranges only from Sulawesi through } \\
\text { Maluku and into Papua }\end{array}$ \\
\hline Ptychosperma & 1 & $\begin{array}{l}\text { (E) Center of diversity is in Papua, with } \\
\text { just a couple other species in the W. Pacific } \\
\text { and Maluku }\end{array}$ \\
\hline Rhopaloblaste & 1 & $\begin{array}{l}\text { (D) Disjunct distribution in that only one } \\
\text { species occurs in the Malay peninsula, and } \\
\text { all other taxa occur East of WL }\end{array}$ \\
\hline Salacca & 1 & $\begin{array}{l}\text { (W) All diversity in the genus is } \\
\text { distributed West of WL, with one taxon } \\
\text { either native or naturalized East of WL }\end{array}$ \\
\hline
\end{tabular}




\begin{tabular}{|l|l|l|}
\hline Genus & $\begin{array}{l}\text { Number of } \\
\text { Moluccan } \\
\text { species in genus }\end{array}$ & $\begin{array}{l}\text { Notes on the distribution of the } \\
\text { genus and centers of diversity in } \\
\text { Malesia }\end{array}$ \\
\hline Saribus & 1 & $\begin{array}{l}\text { (B) Relatively wide range from Borneo in } \\
\text { the West and Raja Ampat islands in the } \\
\text { East, with Papua having the highest species } \\
\text { diversity of the genus }\end{array}$ \\
\hline
\end{tabular}


CHAPTER II

SEED DISPERSAL SYNDROMES OF TROPICAL TREES IN WALLACEA, AND AN EXAMINATION OF THE PAPUAN HORNBILL'S ROLE IN SEED DISPERSAL 


\section{ABSTRACT}

Tropical ecologists have called for prioritizing the protection of seed dispersal processes, especially for the frugivores that provide most of the seed dispersal services for tropical tree species. Their dispersal services affect plant community assembly, persistence, and gene flow in the short-term, and in the long-term are critical to ensuring that tropical trees can regenerate in disturbed areas and migrate amidst climate change. Efforts to conserve keystone dispersers and trees, and to restore mutualisms that aid landscape-scale tree regeneration, are impeded in biodiversity hotspots lacking information. My study aimed to generate inferred information on seed dispersal of tropical trees for one such datascarce island archipelago (the Moluccan Islands of the Wallacea biodiversity hotspot, Indonesia), and examine experimentally the role of a hypothesized keystone disperser (the Papuan hornbill, Rhyticeros plicatus ruficollis). To achieve this aim, I asked: 1) What are the proportions of the varied dispersal syndromes amongst tropical trees and palms in the Moluccan Islands? 2) Is there evidence that a potential disperser, the Papuan hornbill, does actually ingest seeds of trees inferred to be of zoochorous syndrome? If so, then 2a) how many and which plant species does the Papuan hornbill ingest? 2b) is there evidence that the tropical trees being eaten by hornbills are in fact being effectively dispersed? Study results demonstrate that the vast majority of Moluccan tree and palm species are dispersed by frugivores; of all the species dispersed by frugivores, $\sim 35 \%$ rely in part upon the dispersal services of large-bodied frugivores, and an estimated $20 \%$ of the species with zoochorous dispersal syndromes appear to rely exclusively on large- 
bodied frugivores. Of the endemic species, most (77.4\%) are dispersed by frugivores, and of these $29 \%$ appear to be dispersed exclusively by large-bodied frugivores. Papuan hornbills ingest an estimated 10\% of Halmahera Island's tree and palm species, and most of the 35 hornbill-ingested species that were trialled germinated, indicating that Papuan hornbills are indeed effective keystone seed dispersers. Study results emphasize the importance of halting the hunting of large-bodied frugivores to conserve seed dispersal processes that support the region's current and future forests. The study also adds support to the recent calls that functionally important seed dispersers be targeted by conservation efforts not only to sustain their minimum viable populations, but to sustain their populations in a way that conserves their ecological functionality as dispersers.

\section{INTRODUCTION}

Many ecological processes determine how species and communities fare within changing environments. With specific regard to plant-animal interactions, perhaps the most significant of these ecological processes are dispersal, herbivory, pollination, predation, and soil microbial interactions (Herrera and Pellmyr 2009, Kearns et al. 1998, Wardle et al. 2004). Recently, leading tropical ecologists have posited that far greater attention needs to be paid to prioritizing the protection of seed dispersal processes in particular (Howe 2016 and 2014, McConkey et al. 2012). These recent calls for emphasis on examination and conservation of dispersal processes in the tropics are summarily based on two reasons: first, because most tropical trees are dispersed by animals (Howe and Smallwood 1982, Dennis et al. 2007) and those dispersal services affect immediate 
persistence and gene flow (Howe 2014), and second, because seed dispersal dynamics have far-reaching spatio-temporal implications on genetic diversity and migration potential in the face of climate- and environmental- change (Howe 2016, Corlett 2009).

The last two decades have undergone a great deal of progress in seed dispersal research (Traveset et al. 2014), yet gaps in knowledge for many tropical biomes remain. For example, some highly biodiverse islands remain lacking in practical information that will aid in the conservation of their seed dispersal systems; and on a regional scale, many such islands fall within broader areas where data are also limited. Such is the context for our study site of Halmahera island of the Moluccan islands, a geologically complex archipelago within the Wallacea Biodiversity Hotspot (Myers et al. 2000). To help fill knowledge gaps, conducting examinations of certain plant traits - in our case, of tropical trees and palms - may help in predicting species' seed dispersal syndromes and hence provide insights into important seed dispersal interactions and processes (Thomson et al. 2010). Such information can be used for further analyses and applied work in both conservation and restoration planning (Kaiser-Bunbury et al. 2010, Howe 2016, Kuhlman and Ribeiro 2016). While their usefulness is clear, caveats to the practical usage of dispersal syndromes do exist: one of three main categories of potential caveats identified in a recent review by Howe (2016) is the "assum[ption] that use of fruit resources by fruit-eating animals implies effective seed dispersal.” Our study aims not only to examine seed dispersal syndromes amongst the tropical trees and palms in the Moluccan islands, but also to address this caveat by learning about the actual dispersal efficacy of a 
potential disperser, the Papuan hornbill (Rhyticeros plicatus ruficollis) (Fig.2). These aims were achieved by conducting an examination of traits of tropical tree and palm species in order to categorize their inferred dispersal syndromes; then a field study of the actual seed species dispersed by the frugivorous Papuan hornbill was carried out, and lastly we took a preliminary look into whether a subset of the hornbill-dispersed seed species were indeed being effectively dispersed, via a germination trial.

Plant traits \& seed dispersal dynamics in a conservation context

Many modes of dispersal are known to operate in tropical rainforests, including zoochory (dispersal by vertebrates- especially birds, mammals, and reptiles- through ingestion or attachment), autochory (primarily gravity-dispersed or with minimal mechanics), anemochory (dispersal by wind), hydrochory (dispersal by water), myrmecochory (dispersal by ants), and ballochory (dispersal by mechanical ballistics, a form of autochory). Plant phenology and several functional plant traits that have developed through long-term evolutionary processes are associated with certain modes of dispersal (Van der Pijl 1982, Thomson et al. 2010, Howe 2016). Several of the functional traits predominantly linked to dispersal of tropical trees are diaspore size; fruit color, odor, fleshiness, dehiscence, and presentation; and plant habit, in this case tree height (Traveset et al. 2014, Lomáscolo et al. 2010, Corlett 1998, Van der Pij1 1982). Dispersal syndromes that can be inferred through examination of the aforementioned traits can then be associated with potential dispersal distances (again, with caution, as other plant lifehistory and abiotic factors affect this). In Tropical East Asia different categories of 
dispersal vectors have been associated to a coarse level with 'routine maxima' of plant dispersal distances (Corlett 2009).

Ecologists and early naturalists have long perceived the importance of the dispersal services provided by birds, bats, and many other frugivores (e.g., Rumphius 1741, Wallace 1869, Howe and Smallwood 1982, Wheelwright 1985, Loiselle and Blake 1991), yet as Traveset et al. (2014) noted, detailed information on "entire species assemblages of plants, seed dispersers and their interactions is still rarely available." Therefore, efforts to conserve dispersal functionality in habitats and across landscapes, and restore mutualisms (or at minimum, "keystone" dispersers and trees) in disturbed or recovering areas, are generally lacking in important information. It is, however, widely considered that dispersers that enable seeds to travel longer distances, e.g., large-bodied birds and bats, are especially important for protecting landscape-level processes (Corlett 1998) and may have low functional redundancy in some island communities (McConkey and Drake 2015). Evidence also suggests that large-fruited plant species are more susceptible than small-fruited plant species to declines in large-bodied frugivores (e.g., Wotton and Kelly 2011, Mueller et al. 2014). Long distance dispersal by large-bodied frugivores is important for island plants, considering the spectrum of environmental scenarios encountered in the Moluccan islands. At the "relatively intact" end of the spectrum (such as protected forests), it aids them to recruit into areas away from their parent trees, thus avoiding density-dependent mortality (Janzen 1970) and persist in maintaining plant population dynamics and ongoing community assembly. In "disturbed" landscape 
matrices (such as forest fragments, secondary growth, fallow areas), large-bodied frugivores are key to regenerating woody growth in recovering areas that may otherwise remain diversity-limited (Howe and Miriti 2004), and moving diaspores (and hence genetic material) between fragments (Corlett 2009). In the face of climate change, longdistance dispersal is also likely a critical factor in ensuring that plant populations are able to migrate effectively (Howe 2016).

\section{Hornbills as important large-bodied dispersers of zoochorous trees}

Hornbills are iconic examples of large-bodied frugivores. While hornbills are widely considered to be agents of effective seed dispersal (e.g., Holbrook 2002, Kinnaird and O'Brien 2007), a broad and detailed review of hornbill literature suggests that only a few studies to date have examined the potential viability of seeds defecated or regurgitated by hornbills (Kitamura 2011). When considering seed dispersal effectiveness (or "SDE") one may examine elements within two major components of the dispersal service: quantity and quality (Schupp et al. 2010). Germination potential, i.e., viability of dispersed seeds, is an important part of the variables affecting overall "quality" of seed deposition. Across all hornbill species only a small number of studies have demonstrated that seeds defecated or regurgitated by hornbills are able to germinate and, in some cases, exhibit enhanced germination compared with control seeds (e.g., Whitney et al. 1998, Datta 2001, Balasubramanian and Maheswaran 2003, Kitamura et al. 2008, Santhoshkumar and Balasubramanian 2011). 
In order to contribute both broad information on regional plant ecology in Wallacea as well as species-specific information, we asked these questions: 1) what are the proportions of the varied dispersal syndromes amongst tropical trees and palms in the Moluccan Islands? 2) Is there evidence that a potential disperser, the Papuan hornbill, does actually ingest seeds of trees inferred to be of zoochorous syndrome? If so, then 2a) how many and which plant species does the Papuan hornbill ingest? $2 b$ ) is there evidence that the tropical trees being eaten by hornbills are in fact being effectively dispersed? Long-term studies that seek to learn if dispersed seeds ultimately result in a reproductive adult plant are desirable (Schupp et al. 2010) but outside the scope of the current study.

\section{MATERIALS AND METHODS}

\section{Study site}

Within Malesia, our area of interest is that of the Moluccan islands, which lie within the Wallacea hotspot (while not nearly as well inventoried as neighboring areas in Wallacea such as Sulawesi). The entire region of Wallacea is thought to be habitat for approximately 900 - 1000 genera of vascular plants (Marsh et al. 2009), including all plant life forms (e.g., trees, palms, epiphytes, herbs, shrubs, vines). Our study is limited to the Moluccan islands, which occur within the Wallacean biogeographical context, and includes a subset of 317 genera of tropical trees and palms, encompassing approximately 902 tree and palm species that are inferred extant in the Moluccan islands (see below for inference methods). 
Halmahera is the largest island within the Moluccan islands (which span the Indonesian provinces of North Maluku and Maluku). It is recognized not only as a high-biodiversity area being within the Wallacea Biodiversity Hotspot (sensu Myers et al. 2001) (Fig. 1) but also as a high priority area for bird conservation, as it contains several "Important Bird Areas" (sensu BirdLife International 2016). Halmahera contains the only National Park within its geopolitical region of North Maluku. Despite the island's significant biological importance, Halmahera's plant and animal diversity and distributions have barely been mapped, and studies in plant-animal interactions do not yet exist for Halmahera. Further, because of a suite of factors, Halmahera and other islands in the Moluccas have retained very high percentage of forest cover with large areas still containing intact primary forest conditions (e.g., undisturbed by logging, hunting, or conversion) (M. Abdo, unpublished data). Thus there is great need for further scientific examination of Halmahera's ecology to help inform conservation priorities and restoration approaches specific to the island and its National Park.

\section{Study species}

The Papuan (or Blyth's) hornbill, Rhyticeros plicatus ruficollis, is the only hornbill species native to Halmahera. It is one of six subspecies with the easternmost range of $R$. plicatus taxa occuring in the Solomon Islands (Clements et al. 2016). It is a large frugivorous bird approximately $85 \mathrm{~cm}$ in length and up to $2000 \mathrm{~g}$ in body mass (del Hoyo et al. 2001). Papuan hornbills are relatively common, although declining, throughout the island of Halmahera (M. Abdo, unpublished data). Papuan hornbills on Halmahera are 
found both in and adjacent to primary and secondary forests, in "ecotones" and disturbed areas between agroforests, in fallow lands, and in alluvial zones (M. Abdo, unpublished data).

Moluccan tree \& dispersal database

I compiled a database of tropical trees and palms likely to occur in the Moluccan Islands via the best available sources, utilizing the Tree Flora of Indonesia Checklist for Maluku (Whitmore et al. 1989) as the primary source and complementing this with georeferenced records for Maluku from online herbaria resources (e.g., Tropicos) and by querying Kew's World Checklist of Selected Plant Families (WCSP 2017) for the botanical country of Maluku. FileMaker Pro software was used for building and querying the database.

Plant functional traits and dispersal mechanism information (where available) were gathered for each species in the database from both published and unpublished data utilizing detailed diaspore and dispersal data where available (e.g., Smith et al. 2015 for taxa in the Dipterocarpaceae) and utilizing general sources such as LaFrankie (2010) and Flora Malesiana series (2017) for general diaspore characteristics, with Whitmore et al. (1989) providing most attribute data for plant habits and heights, and supplementary attribute data gathered from field observations and other references. Field observations were made over a period of approximately 14 months in Halmahera island and included the collection of diaspore and seed size data using digital calipers (at least 10 diaspores/ 
seeds were measured for each taxon observed, with an averaged measurement reported). Our approach is expected to generate reliable data because intraspecific variability of the plant traits examined, namely seed size, fleshiness, color, and other key dispersal-relevant traits, are not typically significantly plastic within a species (Albert et al. 2011). We chose five dispersal syndrome categories to encompass all potential syndromes in our area, also taking into account associated dispersal distances of related syndromes (Table 1): 1) anemochory, 2) "general" autochory, including ballistic dispersal and/or myrmecochory, 3) hydrochory, 4) zoochory "small-medium," and 5) zoochory "large." For the second category, the term "general" autochory was used as some typically gravity-dispersed tree taxa, e.g., dipterocarps, may sometimes be wind dispersed although they are unlikely to travel far (Smith et al. 2015); myrmecochory was lumped here as 1) data on regional plant taxa that are ant-dispersed are lacking, possibly because myrmecochory is uncommon in tropical trees of this area and 2) ant-dispersed seeds typically have a similar dispersal distance to that of unassisted (large) diaspores (Corlett 2009). For the zoochorous categories, there is some evidence that large diaspores are dispersed by a limited number of large-bodied dispersers (e.g., Wotton and Kelly 2011, Kitamura et al. 2004). Therefore distinctions were made in the attribute data so that the dispersal of large diaspores could be examined. Every species in the database was then assigned a dispersal syndrome category, on the basis of diaspore size and other aforementioned characteristic traits, and on tree height (Table 1 and Fig. 3). Since dispersal syndromes are not mutually exclusive more than one syndrome could be 
assigned to a given taxon, where applicable. In theory, general autochory is a dispersal strategy that may be used by all plants (i.e., their mature diaspores simply fall to the ground in the vicinity of the parent plant), but it was not listed for all taxa, rather only for those taxa that appear to rely on this as a primary or secondary dispersal strategy in comparison to other syndromes. Additional attribute data for each taxon was collected when possible e.g., conservation status, regional endemism, poorly known species or genera (i.e., one or few collections, regionally speciose genera with one or few species described for Maluku), and specific distribution/occurrence data.

\section{Statistical analyses}

A "primary dispersal syndrome" was utilized in the database in order to assign only one dispersal syndrome category (using functional plant traits and available references, we inferred the dispersal syndrome most closely corresponding to the plant's functional features) to trees and palms that had more than one dispersal syndrome category associated with them. For this "primary dispersal syndrome" analysis, one of only three dispersal syndrome categories was assigned to each plant: 1) abiotic dispersal, including gravity or ballistic modes (autochory), hydrochory, and anemochory; 2) zoochory by small- to medium- bodied frugivores; and 3) zoochory by large-bodied frugivores. Data were then analyzed to determine whether there was a significant difference in the proportions of plant families associated with each primary dispersal syndrome, using a chi-square test of independence (McDonald 2014) with a significance of 5\%. 
For the germination trial analysis, seeds were collected from seed traps that captured the freshly-excreted seeds from roosting Papuan hornbills; all collected seeds were categorized into five seed size classes: 1) less than $10 \mathrm{~mm}$; 2) $10-19 \mathrm{~mm}$; 3) $20-29 \mathrm{~mm}$; 4) 30-39mm; and 5) over 40mm. Analysis was carried out using a chi-square test of independence (McDonald 2014) to determine if there was a significant difference in the proportions of seed species that germinated that were associated with different seed species' size classes, with a significance of $5 \%$.

Papuan hornbill dispersal study: observation, seed collecting, and identification

Seeds were collected from hornbill- excreted and regurgitated seeds collected in seed traps below roosting sites. Three known hornbill roosting locations in three different habitats (mixed agroforest, primary forest contiguous with a protected area, and primary forest within a mixed land-use landscape) were surveyed over 12 months in order to capture hornbill-digested seed samples. Hornbills themselves were not handled nor their roosting trees disturbed in any way during the course of this study. Roosting areas consisted of tall emergent trees, one per habitat type, and were frequented by at least 15 hornbills each (on a recurring basis) and occasionally up to 46 individuals simultaneously. One sampling location was located adjacent to the Aketajawe block of the Aketajawe-Lolobata National Park in east Halmahera in primary forest habitat; one sampling location was within a community agroforest/secondary forest matrix in central Halmahera; and one was located within unprotected primary forest in north Halmahera, adjacent to mixed land uses. Each location was visited a minimum of 4 times during the 
12-month period to survey hornbill roosting activity during consecutive 5-day watches. (Note: three other roosting locations were originally chosen for the study in 2011, and additional roosting locations were documented and observed, however by 2014 hornbills had been extirpated from these sites because of hunting, a relatively new local phenomenon discussed later). Six-hour watches of two palm species, Pigafetta filaris and Saribus rotundifolius (two individuals of each species), were conducted three times at each individual (in total 72 observation hours), and hornbill visitations and fruit consumption per visitation was quantified in order to gain a basic estimate for the mean number of fruits that are potentially consumed by Papuan hornbills during their visitation to any individual fruit source.

Sampled collections of hornbill-digested seeds were captured in traps, collected once every five days or on a daily basis when field conditions permitted, i.e., when there was not constant heavy rainfall or flooded impasses. All seeds (across all plant families) were sorted and identified at least to the level of genus and/or by vernacular names, and when identification was not possible seeds were categorized into morpho-species (e.g., Seed A, etc.). Measurements of each seed found in hornbill excrement was collected using digital calipers and seed taxa were sorted into six seed size classes: $<10 \mathrm{~mm}, 10-19 \mathrm{~mm}$, 20-29mm, 30-39mm, 40-49mm, and $>50 \mathrm{~mm}$.

An ex-situ germination trial of 35 hornbill-digested seed taxa (i.e., 35 plant species) was also undertaken. All seed taxa from seed classes over $10 \mathrm{~mm}$ in size were used for the trial, in addition to several palm taxa with seeds less than $10 \mathrm{~mm}$ in size. One hundred 
seeds of each taxon were included in the germination trial. No controls or comparisons were utilized in this germination as a result of field limitations (i.e., insufficient time in the field to locate and collect mature control seeds for all taxa in the study); only hornbilldigested seeds were planted to examine whether or not they germinated. Planting conditions consisted of sterile media in plastic trays kept consistently moist, protected from heavy rainfall with shade cloth and transparent plastic, and otherwise open to the elements with ambient humidity consistently $>85 \%$, in a field nursery located nearby the study sites.

\section{RESULTS}

Dispersal syndromes of tropical trees in the Moluccan islands

A total of 902 tree and palm species encompassing 84 families and 317 genera was enumerated for the Moluccan islands. The 16 most diverse tree families in the region (Table 2) are Moraceae, Euphorbiaceae, Arecaceae, Meliaceae, Phyllanthaceae, Sapotaceae, Fabaceae, Myrtaceae, Araliaceae, Anacardiaceae, Myristicaceae, Ebenaceae, Sapindaceae, Sterculiaceae, Burseraceae, and Rubiaceae. Among them, they contain about $61 \%$ of the total known species diversity of the trees of the Moluccan islands. All of the most diverse families include species that are dispersed by frugivores.

Indeed, the majority (78\%) of Moluccan tree and palm taxa is dispersed via zoochory

(Fig. 4), with zoochory being a primary form of seed dispersal for species from within $69 \%$ of the genera and $93 \%$ of the families. Of all the species dispersed by frugivores, 
about $35 \%$ rely in part upon the dispersal services of large-bodied frugivores. An estimated $20 \%$ of the species with zoochorous dispersal syndromes appear to rely exclusively on large-bodied frugivores, such as Papuan hornbills and large-bodied fruit bats (because of the large diaspore sizes that limit the range of animals that are able to handle or ingest them while keeping their seeds mostly intact). Of the trees and palms dispersed by frugivores of all sizes, about $83 \%$ are exclusively dispersed by frugivores; in other words, they did not appear to have other dispersal syndromes. Anemochory, dispersal by wind, appears to to be the mode of dispersal for approximately $8 \%$ of the Moluccan trees. Hydrochory closely follows this with about $7 \%$ of trees and palms producing diaspores that may be carried by water. In our case this includes the freshwater rivers that are common in the region or the sea (although further studies to determine viability of all hydrochorous seed species after submergence in either salt/fresh water are desirable). General autochory, here including ballistic dispersal as well as dispersal of large seeds with inefficient wing structures (e.g., Dipterocarpaceae), was found in approximately $5 \%$ of species. The proportion of plant families associated with each primary dispersal syndrome differed significantly (chi square $=729.01, \mathrm{df}=166, P<$. 0001).

The regionally endemic species represented about $16 \%$ of the total species we studied and 147 total tree and palm species from 51 genera and 35 families. Of the endemic species, most (77.4\%) are dispersed by frugivores, of these $29 \%$ appear to be exclusively dispersed by large-bodied frugivores. By far, zoochory was the most important dispersal 
syndrome amongst the endemic trees and palms of the Moluccan islands, with autochory coming in a distant second with approximately $12.9 \%$ of the endemics classified as such. Abiotic dispersal via wind or water were also found in endemic species, with $5.6 \%$ and $2.4 \%$ of species using these dispersal mechanisms.

\section{Seed species dispersed by the Papuan hornbill}

Study of the seeds consumed by Rhyticeros plicatus ruficollis yielded at least 71 distinct plant taxa from at least 22 plant families identified in the excrement of the Papuan hornbill (Table 3) from three locations on the island of Halmahera. The primary forest habitat site contiguous with the Aketajawe block of the National Park had the highest number of distinct species of food plants in the Papuan hornbill diet (68 taxa, Table 3), followed by the primary forest contiguous with unprotected habitat and mixed-use areas (57 taxa), and the community agroforest/secondary forest site contained seeds from 37 hornbill food plants (Fig. 5). Table 3 indicates the habitat for each taxon.

Of the plant taxa ingested by the hornbill, I was able to determine 54 to either local vernacular name, botanical plant family, or genus. An additional 17 taxa were identified as distinct morpho-species on the basis of their seed morphology. Of all the fruits consumed by hornbills, about half (50\%) yielded seeds with diameters/lengths less than $10 \mathrm{~mm}$ in size. Seeds in the size class of $10-19 \mathrm{~mm}$ composed $32 \%$ of the total seed species collected, followed by the $20-29 \mathrm{~mm}$ size class consisting of about $12 \%$ of the total seed species. Seed over $30 \mathrm{~mm}$ in size represented about $6 \%$ of the seed species 
ingested by the Papuan hornbill with only 2 seed species falling into the $30-39 \mathrm{~mm}$ size class, and 1 taxon each in both the 40-49mm size class and $>50 \mathrm{~mm}$ size class (see Fig. 6).

Dispersal syndromes of hornbill dispersed species

All of the identified seed species observed as being dispersed by Papuan hornbills were indeed among the species inferred to be zoochorous according to the database we compiled. Eighty-four percent of them are considered to be exclusively dispersed by a range of frugivores. Of these, approximately $10 \%$ were predicted to only be dispersed by large-bodied frugivores. An estimated $16 \%$ of the seed species being dispersed by Papuan hornbills were also classed within abiotic dispersal syndromes i.e., anemochorous, hydrochorous, and/or autochorous dispersal syndromes.

\section{Quantity of fruits ingested during palm visitations}

Papuan hornbills were observed carrying out 5 separate feeding visitations to two different palm species over the course of the 72 hours of observation, with the average number of palm fruits consumed per visitation per hornbill being $145.2( \pm 48.8 \mathrm{SD})$.

\section{Hornbill dispersed seed germination trial}

In the germination trials, most $(29$, or $83 \%)$ of the seed species germinated successfully (Table 3), with all 29 of 35 seed species experiencing at least $50 \%$ germination. The proportion of seed species that germinated was not significantly associated with different seed species size classes (chi square $=3.295 ; \mathrm{df}=4, P=0.51$ ). 


\section{DISCUSSION}

Frugivores are critical in dispersing Moluccan trees and palms

Our results support the view that the Moluccan islands are one of Wallacea's highbiodiversity areas in terms of floristic diversity. A total of 902 tree and palm species encompassing 84 families and 317 genera was enumerated for the Moluccan islands. On the basis of the results of our study, we estimate Halmahera island to contain at least 700 tree species. Halmahera is about $1,000 \mathrm{~km}^{2}$ larger than the total land mass of the Hawaiian Islands, which in comparison are estimated to contain about 1,400 native plant species (including all vascular plants e.g., trees, shrubs, herbs) (Division of Forestry \& Wildlife, State of Hawaii, unpublished data, 2017). Estimates of tree or plant species diversity are unavailable for other Indonesian islands similar in size to Halmahera island.

Ours is the first study to review the dispersal syndromes at work amongst the trees of the Moluccan islands. We found that the majority of the archipelago's trees, and nearly $80 \%$ of the region's endemic species, rely upon the dispersal services of frugivores. Our results support the widely-held perspective that the majority of tropical tree species are dispersed by frugivores (Howe and Smallwood 1982, Dennis et al. 2007). Further, the findings that an estimated $20 \%$ of all trees in the Moluccan islands and nearly $30 \%$ of the region's endemic species rely almost exclusively upon the dispersal services of largebodied frugivores (e.g., hornbills, fruit bats, fruit pigeons) is noteworthy. 
Although there is a far lower percentage of tree species that have "unassisted" dispersal syndromes (e.g., autochory) relative to zoochorous syndromes, some autochoric species may in fact be the abundant trees within various forest habitats within the Moluccas, as in the famous case of Malesia's Dipterocarpaceae trees whose diaspores auto-gyrate typically within $10 \mathrm{~m}$ of their parent plants (Smith et al. 2015). It is also important to note that autochory, including when diaspores are deposited nearby parent plants, is probably an important mechanism whereby some tree and palm populations persist over time (Donatti et al. 2007, Hubbell 1980).

\section{Papuan hornbills are keystone dispersers}

The combined results of our study point to the Papuan hornbill as a keystone disperser in Halmahera. Our study demonstrates that Papuan hornbills provide dispersal services for over 70 species of tropical trees and palms on Halmahera. Papuan hornbills not only disperse a diverse range of species from many different genera, but they also disperse a variety of plant forms including trees, palms, climbers, and "giant herbs" such as wild bananas. Field observations made during the course of the study found hornbills perching and foraging in the forest canopy and among emergent species as well as in the forest understory (not all hornbill species forage in forest understory e.g., Kitamura et al. 2004). In the case of the palms, hornbills were observed consuming a large quantity of fruits during a single visitation, indicating that, in terms of the quantity side of the "seed dispersal effectiveness" equation (sensu Schupp et al. 2010), they are capable of consuming a significant amount of potential diaspores. Studies of different hornbill 
species have observed far fewer fruits consumed per visit (e.g., Kitamura et al. 2004), and thus the number of fruits consumed per visit varies by hornbill, season, and fruit species. Papuan hornbills were tracked opportunistically (on foot) on four occasions in Halmahera. They traveled more than eleven kilometers from their roosts to foraging locations, but we do not yet have meaningful data on their home ranges or total distances travelled while tracking fruit resources. Studies on African hornbills that have tracked their movement precisely using radiotelemetry show that home ranges can span hundreds of kilometers (Holbrook et al. 2002, Chasar et al. 2014). In Halmahera, Papuan hornbills were observed across a spectrum of intact and disturbed habitats and crossing a variety of such habitats including rural roads (M. Abdo, unpublished data) which may indicate that they play a role in forest regeneration in disturbed areas or forest margins, in addition to dispersing seeds between/within intact forest habitats. Other studies have shown hornbills to track fruit resources (Kinnaird and O'Brien 2007, Naniwadekar et al. 2015, Whitney and Smith 1998) and vary their diets according to breeding season (Lamperti et al. 2014). During our study period, hornbills were extirpated (by hunting) from several study sites in different habitats that were thus not possible to sample properly. On the basis of these factors, we believe that this study's results may be an underestimate of the total plant species that Papuan hornbills consume and disperse within Halmahera. The current study shows that, considering the potential distances travelled by Papuan hornbills, the high number of fruits ingested during a visitation, the large proportion of hornbill-digested dispersed seeds that successfully germinate, and the diversity of tree 
and palm species consumed, Papuan hornbills are effective seed dispersers in the Moluccan islands. Hornbills in Halmahera likely play important roles in forest community assembly as well as forest regeneration, dispersing at least $10 \%$ of the island's tree and palm species.

\section{Species interactions}

While this study provides important new information and analysis of both the tree and palm dispersal syndromes for the Moluccan islands, as well as insights into Papuan hornbills as keystone dispersers for the area, there remain many avenues for exploration into the plant and animal communities and their interactions in Wallacea. A review of Halmahera's frugivores and seed predators is desirable, as are detailed long-term studies of tree and palm seed fates after diaspore interactions with various guild members (e.g., dispersers, scavengers, predators). Helgen (2002) noted in a brief review of museum collections that "mammalian patterns of species richness and distribution in the Moluccas are still obscure," and in 2013 a new genus of terrestrial rodents (with vegetative remains in their stomachs) was discovered in Halmahera (Fabre et al. 2013). In a study site in Thailand, the large-fruited tree Aglaia spectabilis was effectively dispersed by hornbills, with fallen seeds that were not bird-dispersed being heavily preyed upon by rodents and other mammals, and only in a few autochoric cases surviving into seedlings in the vicinity of the parent trees (Kitamura et al. 2004). Early-life seed fates of palms dispersed by hornbills is experimentally investigated in a separate chapter of this dissertation; however, the interactions between guild members and their effects on seed 
fate await investigation in Halmahera and many questions remain. For example, we anecdotally observed scat from below three white cockatoo (Cacatua alba) nesting sites and found evidence that the cockatoos may be seed predators as a result of the way they crush the fruits they ingest, precluding seed viability for most species but possibly permitting it for Ficus spp. and other fruits with small embedded seeds. Interestingly, white cockatoos and hornbills in Halmahera were also found nesting in the same holes in the same tree individuals, but at different times; when cockatoos nested they often snapped branches and carried out "shrub clearing" activity around their nesting trees creating mini-gaps in the forest. When hornbills nest in the same trees subsequently, do these cockatoo-created mini-gaps cause hornbill dispersed seeds to have an increased chance of attaining light and space resources for survival?

There are many mammals and birds whose varied interactions with plants need to be investigated. Halmahera alone harbors at least 265 species of birds, many of which are endemic species, and an estimated 19 of which are species of native fruit doves and pigeons including several large-bodied endemic fruit pigeons (e.g., Ptilinopus and Ducula species, among other genera) (Clements et al. 2015). To our knowledge, a complete checklist of all of Halmahera's bats is not yet available, but there are numerous species of bats that are likely important pollinators and seed dispersers extant on the island, including megabat (i.e., flying foxes and large fruit bats) and microbat taxa. During the course of this study, one unidentified species of megabat was documented dispersing at least three species of very large-fruited trees and palms; in fact, these plant 
species' diaspores were so large (such as the palm Orania palindan) we do not believe they could be dispersed by hornbills (and they were not found in any hornbill seed trap sites). The $O$. palindan fruits were carried over 20 meters from their parent plants by the bats, and three of six bat-dispersed $O$. palindan seeds we trialled in our field nursery germinated. In the case of another palm species with smaller fruits, Rhophaloblaste ceramica, we observed bats dispersing apparently intact seeds (after the fruits' fleshy parts or exocarp had been consumed). Hornbills were also found dispersing this species, albeit depositing the seeds closer to parent plants than hornbills typically do with other palm species.

Detailed investigations are needed in order to begin gaining insights about broader ecological questions for the insular ecologies of the Moluccas (e.g., whether dietary overlap exists for some species, and where does Halmahera fall along the low-to-high dispersal redundancy spectrum?). Several of the differences among insular dispersal guilds in comparison to mainland guilds are briefly reviewed by Traveset et al. (2014) and include broadening or "niche expansion" of diets otherwise not typically frugivorous (Wright 1980), and higher frequency of unusual mechanisms (e.g., secondary seed dispersal). An example of niche expansion is known north of the Moluccas in the Philippines, where survey efforts have documented frugivorous monitor lizards that feed upon palm, fig, and pandan fruits (Siler et al. 2011); congeners of these reptiles are known to occur in the Moluccas but their ecological interactions and dispersal potential is unknown. 


\section{Conservation implications}

Other studies have shown that large-bodied frugivores are very important to ensuring effective dispersal of large-seeded tree species (Wotton and Kelly 2011, Vanthomme et al. 2010) and in some cases may be the only effective dispersers for certain plant species (McConkey and Drake 2015). Studies have also shown that hunting and logging pressures affecting hornbill presence/abundance in turn have effects on seed arrival and recruitment of trees typically dispersed by frugivores (Naniwedekar et al. 2015). In a study of megabats (flying foxes), researchers found that declines in their numbers caused them to become functionally extinct as dispersers prior to the tipping point when their numbers would have delineated them as rare (McConkey and Drake 2006). Our study found that Papuan hornbills are being increasingly hunted in Halmahera, as observed in cases where roost trees previously inhabited by hornbills consistently $>15$ years have recently been hunted out. The decline of hornbills caused by hunting must be halted not only before their numbers become rare, but before the ecologically critical service of dispersal that hornbills provide is impeded.

Most of the diversity of tropical trees is held within small populations of rare taxa (Slik et al. 2015, Howe 2014, Hubbell 2013). Although it was far outside the scope of our study to take into account species abundances for all the tree species included in our analysis, we did learn that the majority of the region's trees and palms - and known endemic and endangered taxa - relies heavily on dispersal services provided by frugivores. In some forest habitats such as those studied within Halmahera, trees and palms dispersed by 
frugivores composed the dominant forest canopy community (M. Abdo, unpublished data). With areas such as the Wallacea biodiversity hotspot (and many other tropical and temperate biomes worldwide) having genuine social and economic constraints impeding efforts to conserving ecosystem functionality at multiple scales (Hooper et al. 2005), it seems that the roles many frugivores play goes beyond that of "forest farmers" and indeed also includes "rare species conservators." Humans do not yet know the identity or phenology or location of many of the world's rare plant species, but frugivores like the Papuan hornbill do, and are actively dispersing and conserving their lineages. Our study results emphasize the importance of earlier pleas made to halt the hunting/poaching of large-bodied frugivores (e.g., frugivorous birds and bats) in particular (Corlett 2009), in order to support the conservation of seed dispersal processes that support the region's current and future forests. Furthermore, our study adds support to the recent calls that functionally important seed dispersers be targeted by conservation efforts not only according to goals to sustain their minimum viable populations, but in order to sustain their populations in a way that conserves their ecological functionality as dispersers (McConkey et al. 2012).

\section{REFERENCES}

Albert, C. H., Grassein, F., Schurr, F. M., Vieilledent, G., \& Violle, C. (2011). When and how should intraspecific variability be considered in trait-based plant ecology? Perspectives in Plant Ecology, Evolution and Systematics, 13(3), 217-225.

BirdLife International. (2016). Important Bird Areas factsheet: Aketajawe. Downloaded from http://www.birdlife.org on 26/11/2016. 
Brown, R. M. (2011). Amphibians and Reptiles, Luzon Island, Aurora Province and Aurora Memorial National Park, Northern Philippines: New island distribution records.

Clements, J. F., T. S. Schulenberg, M. J. Iliff, D. Roberson, T. A. Fredericks, B. L. Sullivan, and C. L. Wood. (2016). The eBird/Clements checklist of birds of the world: v2016. Downloaded from http://www.birds.cornell.edu/clementschecklist/download/ on 26/11/2016.

Corlett, R. T. (1998). Frugivory and seed dispersal by vertebrates in the Oriental (Indomalayan) Region. Biological Reviews, 73(4), 413-448.

Corlett, R. T. (2009). Seed dispersal distances and plant migration potential in tropical East Asia. Biotropica, 41(5), 592-598.

del Hoyo, J., Elliott, A. and Sargatal, J. (2001). Handbook of the Birds of the World. Volume 6: Mousebirds to Hornbills. Lynx Edicions, Barcelona.

Dennis, A.J., Schupp, E.W., Green, R.J., \& Westcott, D.A. (2007). Seed Dispersal: Theory and Its Application in a Changing World. CAB International, Wallingford, UK.

Donatti, C.I., Galetti, M., Pizo, M.A., Guimaraes Jr., P.R., \& Jordano, P. (2007). Living in the Land of Ghosts: Fruit Traits and the Importance of Large Mammals as Seed Dispersers in the Pantanal, Brazil, in Seed Dispersal: Theory and its Application in a Changing World, Eds A.J. Dennis et al.

Graham, L. L., \& Page, S. E. (2012). Artificial bird perches for the regeneration of degraded tropical peat swamp forest: a restoration tool with limited potential. Restoration Ecology, 20(5), 631-637.

Herrera, C. M., \& Pellmyr, O. (Eds.). (2009). Plant animal interactions: an evolutionary approach. John Wiley \& Sons.

Herrera, J., \& Garcia, D. (2010). Effects of forest fragmentation on seed dispersal and seedling establishment in ornithochorous trees. Conservation Biology, 24(4), 1089-1098.

Holbrook, K. M., Smith, T. B., \& Hardesty, B. D. (2002). Implications of long-distance movements of frugivorous rain forest hornbills. Ecography, 25(6), 745-749.

Hooper, D. U., Chapin, F. S., Ewel, J. J., Hector, A., Inchausti, P., Lavorel, S., ... \& Schmid, B. (2005). Effects of biodiversity on ecosystem functioning: a consensus of current knowledge. Ecological Monographs, 75(1), 3-35. 
Howe, H.F., and Smallwood, J. (1982). Ecology of seed dispersal. Annual Review of Ecology, Evolution, and Systematics 13: 201-228.

Howe, H. F. (2016). Making dispersal syndromes and networks useful in tropical conservation and restoration. Global Ecology and Conservation, 6, 152-178.

Howe, H. F. (2014). Diversity storage: Implications for tropical conservation and restoration. Global Ecology and Conservation, 2, 349-358.

Howe, H. F., \& Miriti, M. N. (2004). When seed dispersal matters. BioScience, 54(7), 651-660.

Hubbell, S. (1980) Seed predation and the coexistence of tree species in tropical forests. Oikos 35, 214-229.

Hubbell, S.P. (2013). Tropical rain forest conservation and the twin challenges of diversity and rarity. Ecology and Evolution 3(10):3263-3274.

Janzen, D. H. (1970). Herbivores and the number of tree species in tropical forests. American Naturalist. 104: 501-528.

Jordano, P., Forget, P. M., Lambert, J. E., Böhning-Gaese, K., Traveset, A., \& Wright, S. J. (2011). Frugivores and seed dispersal: mechanisms and consequences for biodiversity of a key ecological interaction. Biology Letters., 7: 321-323

Kearns, C. A., Inouye, D. W., \& Waser, N. M. (1998). Endangered mutualisms: the conservation of plant-pollinator interactions. Annual review of Ecology and Systematics, $29(1), 83-112$.

Kinnaird, M. F. and O' Brien, T. G. (2007). The ecology and conservation of Asian hornbills. University of Chicago Press, Chicago, Illinois

Kinnaird, M.F. (1998). Evidence for effective seed dispersal by the Sulawesi redknobbed hornbill, Aceros cassidix. Biotropica 30, 50-55.

Kitamura, S. Suzuki, S.,. . . Chumphon Suckasam. (2004). Dispersal of Aglaia spectabilis, a Large-Seeded Tree Species in a Moist Evergreen Forest in Thailand. Journal of Tropical Ecology, 20(4), 421-427.

Kitamura, S., Yumoto, T., Noma, N., Chuailua, P., Maruhashi, T., Wohandee, P., \& Poonswad, P. (2008). Aggregated seed dispersal by wreathed hornbills at a roost site in a moist evergreen forest of Thailand. Ecological Research, 23(6), 943-952. 
Kitamura, S. (2011). Characteristics of hornbill-dispersed fruits in lowland dipterocarp forests of southern Thailand. The Raffles Bulletin of Zoology, (24), 137-147.

LaFrankie, J.V. (2010). Trees of Tropical Asia: An Illustrated Guide to Diversity. Black Tree Publications, Philippines.

Lamperti, A.M., French, A.R., Dierenfeld, E.S., Fogiel, M.K.,... \& Parker, V. T. (2014). Diet selection is related to breeding status in two frugivorous hornbill species of Central Africa. Journal of Tropical Ecology, 30:273-290.

Lebrija-Trejos, E., Pérez-García, E. A., Meave, J. A., Bongers, F., \& Poorter, L. (2010). Functional traits and environmental filtering drive community assembly in a species-rich tropical system. Ecology, 91(2), 386-398.

Loiselle, B. A., \& Blake, J. G. (1991). Temporal variation in birds and fruits along an elevational gradient in Costa Rica. Ecology, 72 (1), 180-193.

McConkey, K. R., \& Drake, D. R. (2015). Low redundancy in seed dispersal within an island frugivore community. AoB Plants, 7, plv088.

McConkey, K. R., Prasad, S., Corlett, R. T., Campos-Arceiz, A., Brodie, J. F., Rogers, H., \& Santamaria, L. (2012). Seed dispersal in changing landscapes. Biological Conservation, 146(1), 1-13.

McDonald, J.H. (2014). Handbook of Biological Statistics (3rd ed.). Sparky House Publishing, Baltimore, Maryland

Mello, M. A. R., Rodrigues, F. A., Costa, L. D. F., Kissling, W. D., Şekercioğlu, Ç. H., Marquitti, F. M. D., \& Kalko, E. K. V. (2015). Keystone species in seed dispersal networks are mainly determined by dietary specialization. Oikos, 124(8), 1031-1039.

Mueller, T., J. Lenz, T. Caprano, W. Fielder, K. Boehning-Gaese. (2014.) Large frugivorous birds facilitate functional connectivity of fragmented landscapes. Journal of Applied Ecology 51: 684692.

Myers, N., Mittermeier, R. A., Mittermeier, C. G., Da Fonseca, G. A., \& Kent, J. (2000). Biodiversity hotspots for conservation priorities. Nature, 403 (6772), 853-858.

Myers, J. A., \& Harms, K. E. (2011). Seed arrival and ecological filters interact to assemble high-diversity plant communities. Ecology, 92(3), 676-686. 
Naniwadekar, R., Mishra, C., \& Datta, A. (2015). Fruit resource tracking by hornbill species at multiple scales in a tropical forest in India. Journal of Tropical Ecology, doi: $10.1017 / \mathrm{S} 0266467415000449$

Rodríguez-Pérez, J., \& Traveset, A. (2010). Seed dispersal effectiveness in a plant-lizard interaction and its consequences for plant regeneration after disperser loss. Plant Ecology, 207(2), 269-280.

Rumphius, G.E. (1741). Herbarium Amboinense, Netherlands.

Slik, J. F., Arroyo-Rodríguez, V., Aiba, S. I., Alvarez-Loayza, P., Alves, L. F., Ashton, P., ... \& Bernacci, L. (2015). An estimate of the number of tropical tree species.

Proceedings of the National Academy of Sciences, 112(24), 7472-7477.

Shipley, B. (2010). From plant traits to vegetation structure: chance and selection in the assembly of ecological communities. Cambridge University Press.

Spotswood, E. N., Meyer, J. Y., \& Bartolome, J. W. (2012). An invasive tree alters the structure of seed dispersal networks between birds and plants in French Polynesia.

Journal of Biogeography, 39(11), 2007-2020.

Tamme, R., Götzenberger, L., Zobel, M., Bullock, J. M., Hooftman, D. A., Kaasik, A., \& Pärtel, M. (2014). Predicting species' maximum dispersal distances from simple plant traits. Ecology, 95(2), 505-513.

Thomson, F. J., Moles, A. T., Auld, T. D., Ramp, D., Ren, S., \& Kingsford, R. T. (2010). Chasing the unknown: predicting seed dispersal mechanisms from plant traits. Journal of Ecology, 98(6), 1310-1318.

Traveset, A., Heleno, R., Nogales, M., \& Gallagher, R. S. (2014). The ecology of seed dispersal, 62-93 in Gallagher, R. S. (Ed.). Seeds: The ecology of regeneration in plant communities. Cabi.

Vanthomme, H., Bellé, B., \& Forget, P. M. (2010). Bushmeat hunting alters recruitment of large-seeded plant species in central Africa. Biotropica, 42, 672-679.

Wallace, A. R. (1869). The Malay Archipelago: the land of the orang-utan and the bird of paradise; a narrative of travel, with studies of man and nature. Courier Corporation.

Wardle, D. A., Bardgett, R. D., Klironomos, J. N., Setälä, H., Van Der Putten, W. H., \& Wall, D. H. (2004). Ecological linkages between aboveground and belowground biota. Science, 304 (5677), 1629-1633. 
WCSP (2017). World Checklist of Selected Plant Families. Facilitated by the Royal Botanic Gardens, Kew. Published on the Internet; http://apps.kew.org/wcsp/ Retrieved 03 Jan 2017.

Wheelwright, N.T., (1985). Fruit size, gape width, and the diets of fruit-eating birds. Ecology 66: 808-818.

Whitmore, T. C., Tantra, I.G.M., \& Sutisna, U. (1989). Tree flora of Indonesia: Checklist for Maluku. Forest Research and Development Centre, Bogor, Indonesia.

Whitney, K.D. \& T.B. Smith. (1998). Habitat use and resource tracking by African Ceratogymna hornbills: implications for seed dispersal and forest conservation. Animal Conservaton 1: 107-117.

Wotton, D. M., \& Kelly, D. (2011). Frugivore loss limits recruitment of large-seeded trees. Proceedings of the Royal Society of London: Biological Sciences, 278, 3345-3354.

\section{FIGURES AND TABLES}

Figure 1: Map illustrating the Wallacea Biodiversity hotspot containing the Moluccan islands, highlighting Halmahera Island and its Aketajawe-Lolobata National Park

Figure 2: A pair of Papuan hornbills (Rhyticeros plicatus ruficollis), frugivores and seed dispersers, in forest habitat of Halmahera, Indonesia

Figure 3: Examples of tree fruits with zoochorous dispersal syndromes in the Moluccan Islands of Indonesia.

Figure 4: Proportions of tree species in Moluccan Islands of Indonesia across dispersal syndromes.

Figure 5: Number of plant species ingested by Papuan hornbill at each site in Halmahera

Figure 6: Comparison of seed size classes of plant taxa digested by the Papuan hornbill

Table 1: Categories of dispersal syndromes utilized in this study

Table 2: Most diverse tree families of the Moluccan Islands of Indonesia

Table 3: Plant species dispersed by the Papuan hornbill, including notation of species utilized in the germination trial 
Figure 1: Map illustrating the Wallacea Biodiversity hotspot containing the Moluccan islands, highlighting Halmahera Island and its Aketajawe-Lolobata National Park




Figure 2: A pair of Papuan hornbills (Rhyticeros plicatus ruficollis), frugivores and seed dispersers, in forest habitat of Halmahera, Indonesia

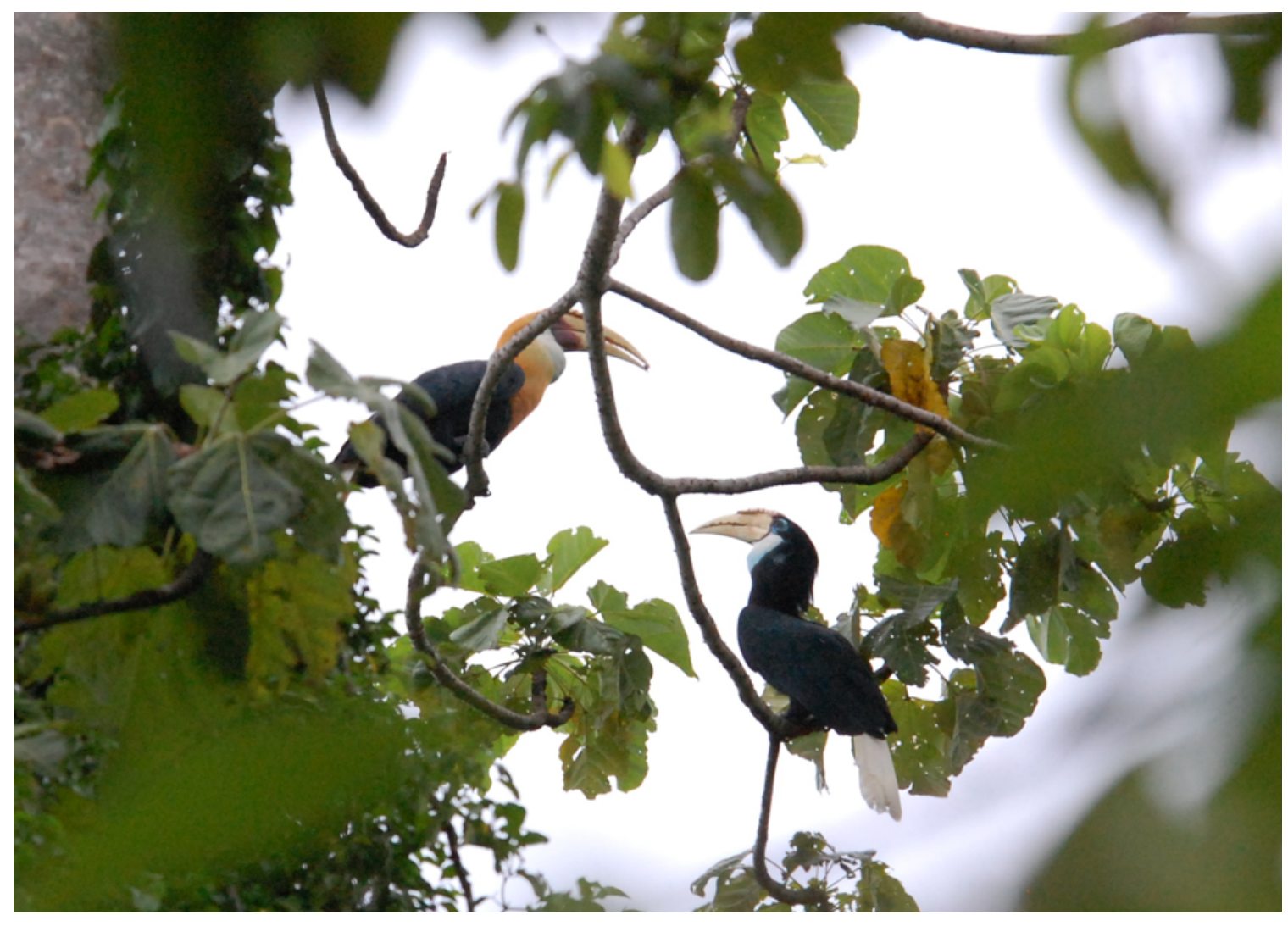


Figure 3: Examples of tree fruits with zoochorous dispersal syndromes in the Moluccan Islands of Indonesia.

(1) Canarium vulgare Leenh. (Burseraceae), note: fruits ripen to nearly black in color; (2) Horsfieldia Willd. sp. (Myristicaceae), (3) Myristica fatua Houtt. (Myristicaceae), (4) and (5) Moluccan endemic Kibara coriacea (Blume) Hook. f. \& Thomson (Monimiaceae), 2 different individuals; (6) Garcinia celebica L. (Clusiaceae); (7) Chisocheton pentandrus (Blanco) Merr. (Meliaceae).

All photos M. Abdo, photographed on Halmahera island.
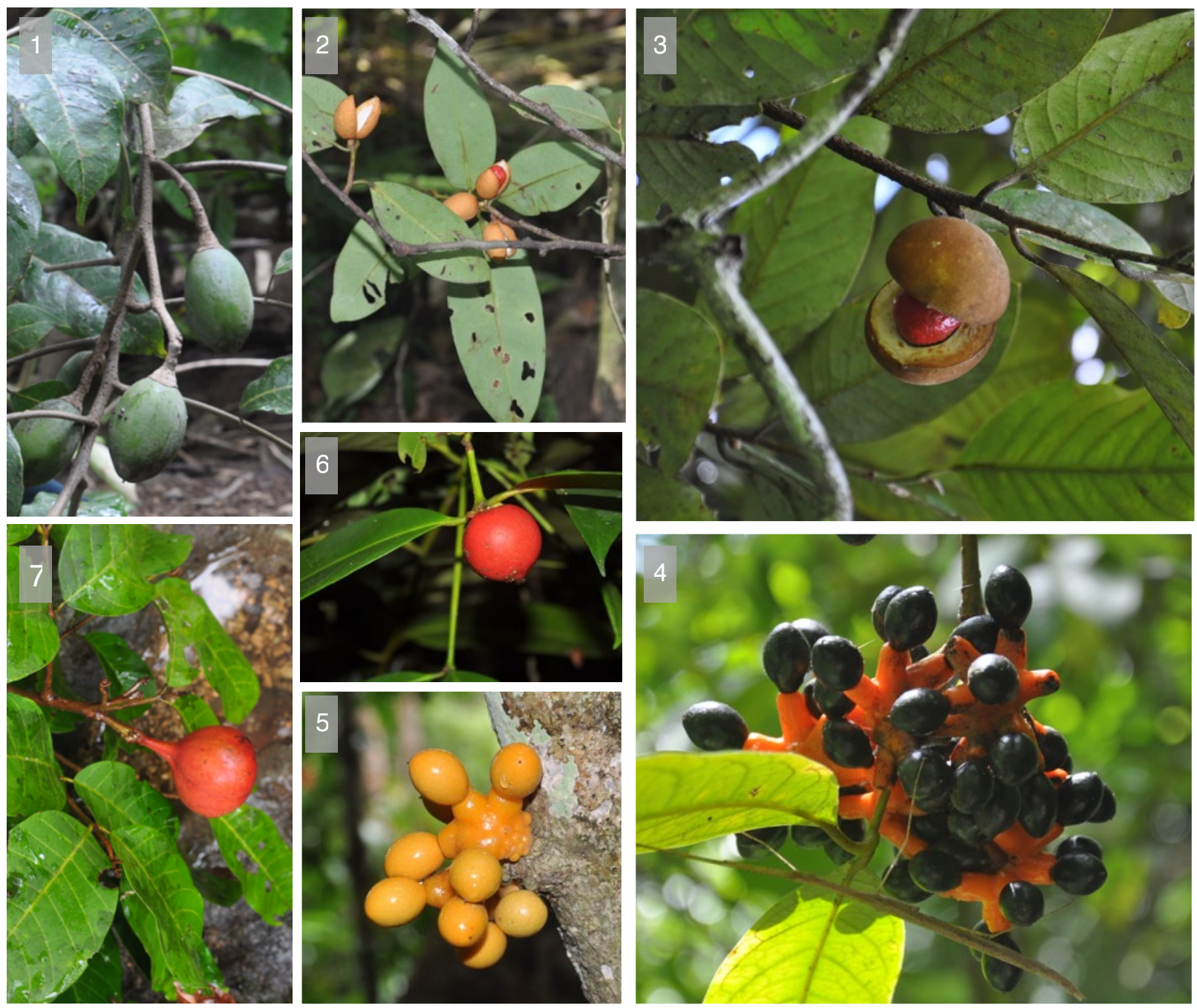
Figure 4: Proportions of Moluccan tree and palm species across dispersal syndromes

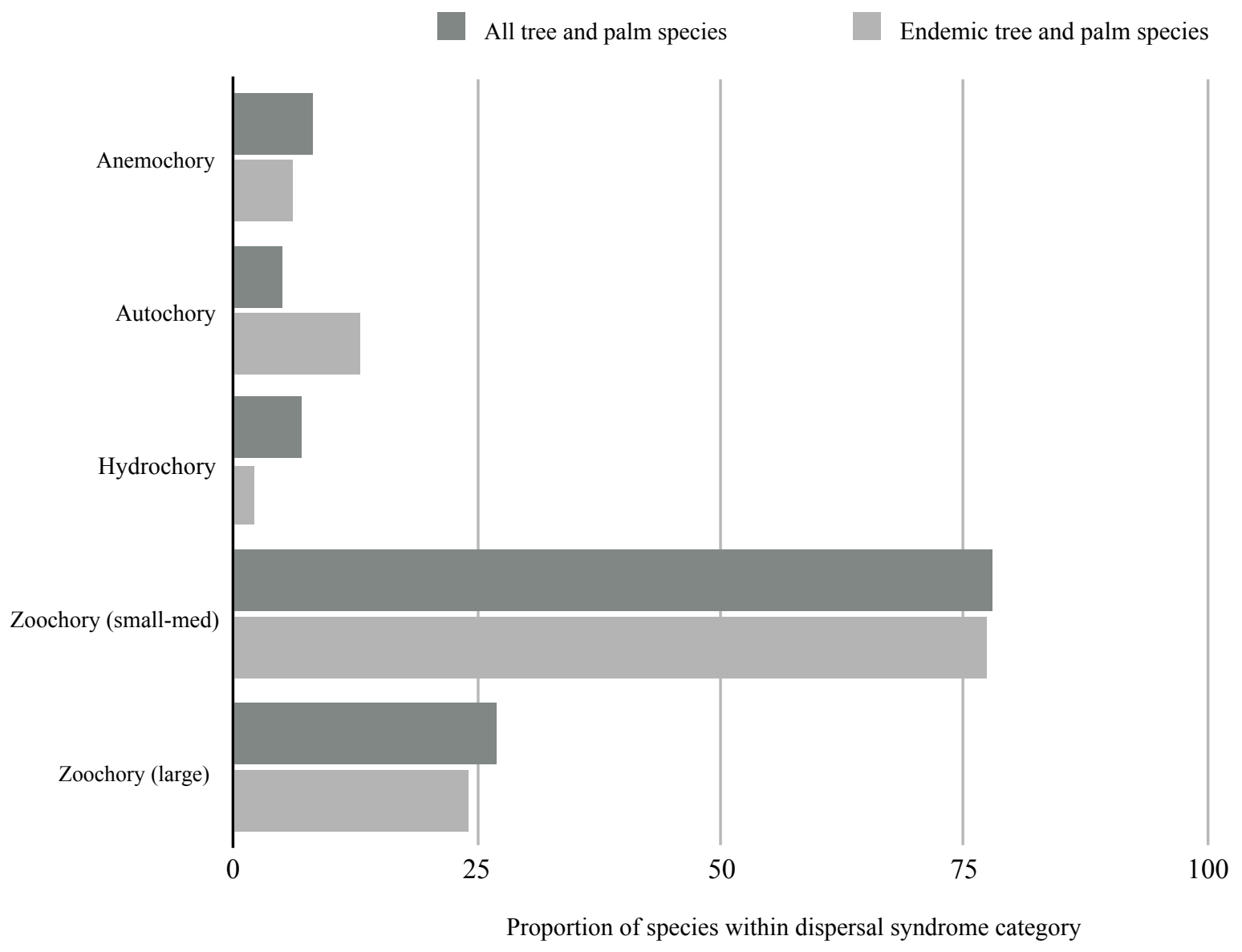


Figure 5: Comparison of seed size classes of plant taxa digested by Papuan hornbills on the Halmahera Island, North Maluku Province (or Moluccan Islands), Indonesia.

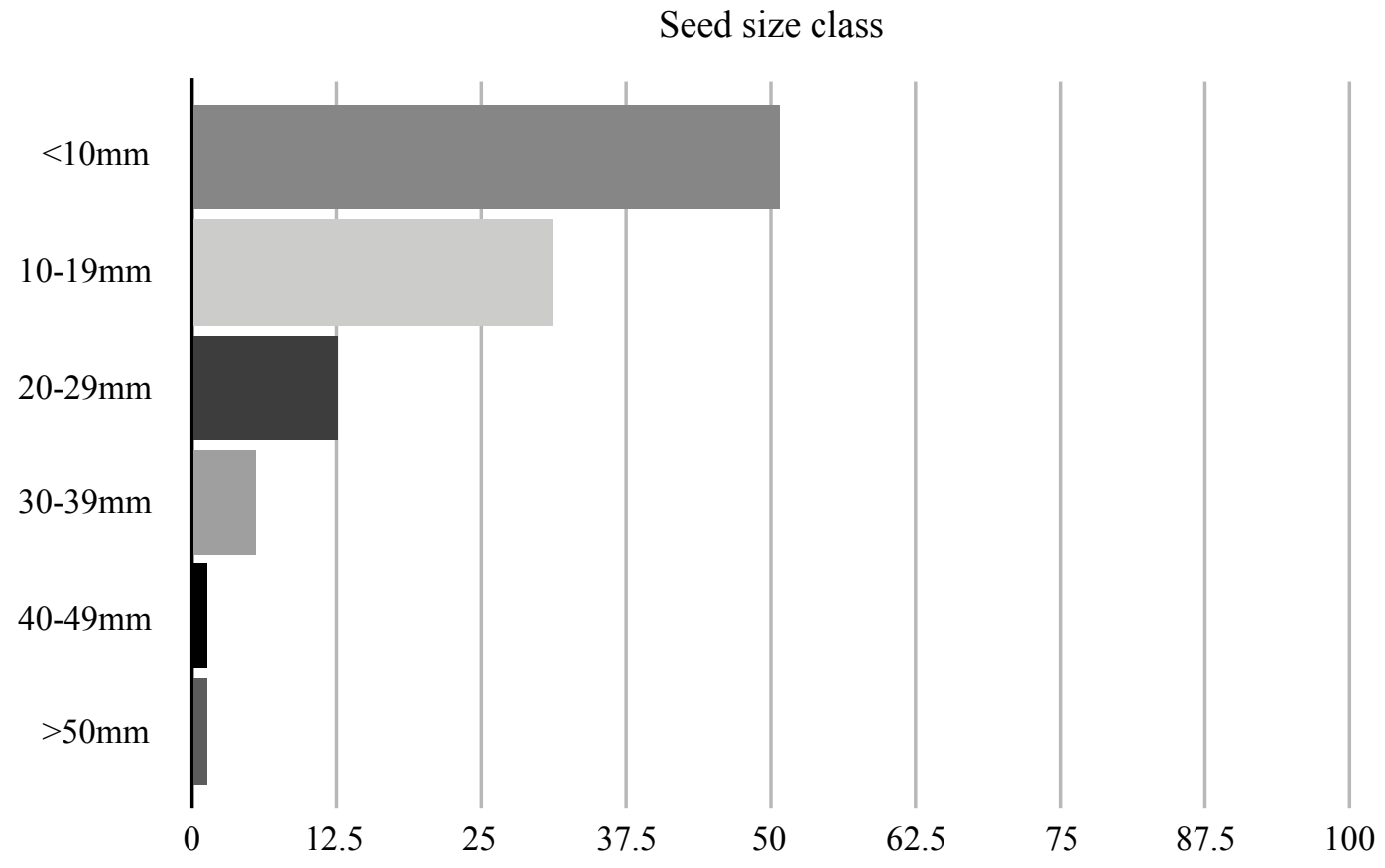

Percentage of plant species occuring in each seed size class 
Figure 6: Number of plant species digested by Papuan hornbills at each site on the Halmahera Island, North Maluka Province (or Moluccan Islands), Indonesia.

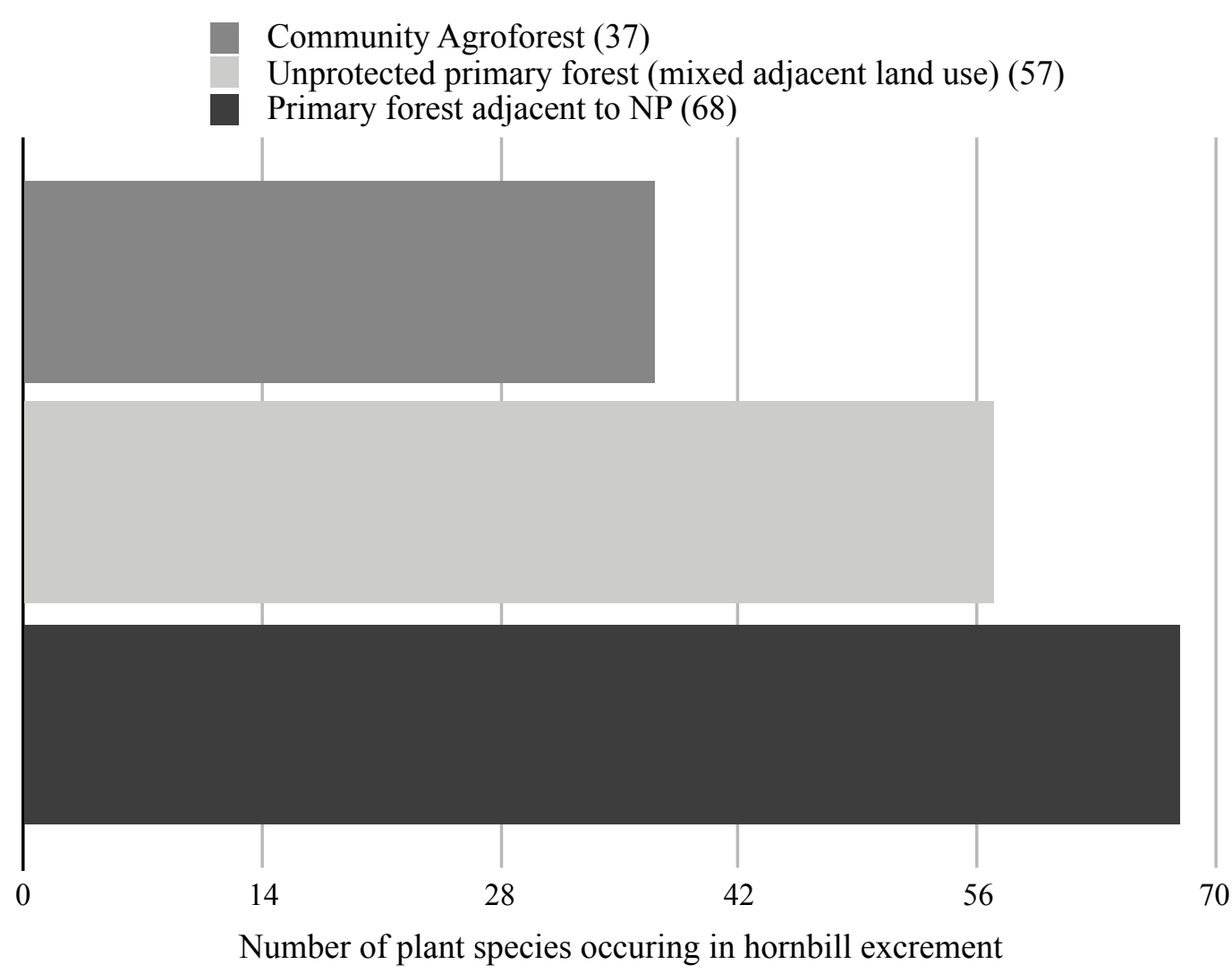


Table 1: Categories of dispersal syndromes utilized in this study

\begin{tabular}{|c|c|c|}
\hline Dispersal category name & Fruit morphology & $\begin{array}{l}\text { Dispersal } \\
\text { distance* }\end{array}$ \\
\hline $\begin{array}{l}\text { Anemochory } \\
\text { Small seeds in wind }\end{array}$ & $\begin{array}{l}\text { Tiny (dust-like) or small seeds }<7 \mathrm{~mm} \text {, or } \\
\text { lighweight (low seed mass) larger-sized seeds } \\
<20 \mathrm{~mm} \text { in size. Fruits of these trees are } \\
\text { sometimes large, but are dehiscent to disperse } \\
\text { their seeds usually from tall plant heights. }\end{array}$ & $>10 \mathrm{~km}$ \\
\hline $\begin{array}{l}\text { General autochory and/ } \\
\text { or myrmecohory } \\
\text { Unassisted seeds of } \\
\text { small-medium size or } \\
\text { large winged auto- } \\
\text { gyrating seeds e.g., } \\
\text { Dipterocarps } \\
\text { Ballistic } \\
\text { Ant-dispersed }\end{array}$ & $\begin{array}{l}\text { Dry and/or typically dehiscent fruits and small } \\
\text { seeds ranging from }>7 \mathrm{~mm} \text { in size to }>20 \mathrm{~mm} \text {. } \\
\text { Includes large winged seeds with and without } \\
\text { wind effect ("auto-gyrating" sensu Smith et al. } \\
\text { 2015). Ballistically dispersed seeds (e.g., as in } \\
\text { some Euphorbiaceae) also included here. } \\
\text { Varied colors, but often brownish. Ant- } \\
\text { dispersed fruits often have a small appendage } \\
\text { with some type of lipid-reward on them. }\end{array}$ & $0-100 \mathrm{~m}$ \\
\hline $\begin{array}{l}\text { Hydrochory } \\
\text { Water dispersed in rivers } \\
\text { or possibly across open } \\
\text { sea }\end{array}$ & $\begin{array}{l}\text { Fruits typically (but not always) large in size } \\
>20 \mathrm{~mm} \text { and with buoyant exocarp or hard } \\
\text { exocarp observed to permit diaspore to float in } \\
\text { either fresh or salt water. }\end{array}$ & $>10 \mathrm{~km}$ \\
\hline $\begin{array}{l}\text { Zoochory, small-med } \\
\text { Small- and medium- } \\
\text { bodied vertebrates } \\
\text { (e.g., many birds, } \\
\text { rodents } \\
\text { many herbivores, } \\
\text { bats with large seeds) }\end{array}$ & $\begin{array}{l}\text { Small and medium sized fruits and seeds, } \\
10 \mathrm{~mm}-25 \mathrm{~mm} \text {, typically with fleshy covering } \\
\text { and/or seeds with colorful aril surrounding } \\
\text { them. Fruits may be displayed amidst foliage, } \\
\text { on infructescences, or cauliflorous. Fruit or } \\
\text { aril colors range from yellow to red, green, or } \\
\text { blue to purple and black and usually mature/ } \\
\text { ripen directly on parent plant. Often } \\
\text { indehiscent. }\end{array}$ & $\begin{array}{l}10 \mathrm{~m}-10 \\
\mathrm{~km}\end{array}$ \\
\hline $\begin{array}{l}\text { Zoochory, large } \\
\text { Large-bodied vertebrates } \\
\text { (e.g., some fruit pigeons, } \\
\text { large fruit bats with tiny } \\
\text { seeds, hornbills) }\end{array}$ & $\begin{array}{l}\text { Large fruits and seeds }>25 \mathrm{~mm} \text {, typically with } \\
\text { fleshy covering and/or seeds with colorful } \\
\text { arils. Fruit or aril colors range from yellow to } \\
\text { red, green, or blue to purple and black and } \\
\text { usually mature/ripen directly on parent plant. } \\
\text { Usually ripen on parent plant, often } \\
\text { indehiscent. }\end{array}$ & $>10 \mathrm{~km}$ \\
\hline
\end{tabular}

*Modified from Corlett (2009) 
Table 2: The 16 most diverse tree families of the Moluccan Islands of Indonesia

\begin{tabular}{|c|c|c|c|}
\hline Plant family & $\begin{array}{l}\text { Total No. } \\
\text { of Taxa } \\
\text { within } \\
\text { family in } \\
\text { Moluccan } \\
\text { islands }\end{array}$ & $\begin{array}{l}\text { Dispersal Syndrome(s) among } \\
\text { Moluccan island species }\end{array}$ & $\begin{array}{l}\text { Papuan } \\
\text { hornbill } \\
\text { dispersal w/in } \\
\text { family on } \\
\text { Halmahera? }\end{array}$ \\
\hline Moraceae & 83 & $\begin{array}{l}\text { - Zoochory, including by large- } \\
\text { bodied frugivores }\end{array}$ & Yes \\
\hline Euphorbiaceae & 59 & $\begin{array}{l}\text { - Zoochory, including by large- } \\
\text { bodied frugivores } \\
\text { - Autochory including ballistic } \\
\text { dispersal } \\
\text { - Anemochory }\end{array}$ & Yes \\
\hline Arecaceae & 50 & $\begin{array}{l}\text { - Zoochory, including by large- } \\
\text { bodied frugivores } \\
\text { - Hydrochory }\end{array}$ & Yes \\
\hline Meliaceae & 50 & $\begin{array}{l}\text { - Zoochory, including by large- } \\
\text { bodied frugivores }\end{array}$ & Yes \\
\hline Phyllanthaceae & 45 & $\begin{array}{l}\text { - Zoochory } \\
\text { - Autochory }\end{array}$ & Yes \\
\hline Sapotaceae & 38 & $\begin{array}{l}\text { - Zoochory, including by large- } \\
\text { bodied frugivores } \\
\text { - Hydrochory }\end{array}$ & Unknown* \\
\hline Fabaceae & 35 & $\begin{array}{l}\text { - Zoochory } \\
\text { - Autochory including ballistic } \\
\text { dispersal } \\
\text { - Anemochory }\end{array}$ & Yes \\
\hline Myrtaceae & 28 & $\begin{array}{l}\text { - Zoochory, including by large- } \\
\text { bodied frugivores } \\
\text { - Autochory }\end{array}$ & Yes \\
\hline Araliaceae & 27 & - Zoochory & Unknown* \\
\hline Anacardiaceae & 25 & $\begin{array}{l}\text { - Zoochory, including by large- } \\
\text { bodied frugivores }\end{array}$ & Unknown* \\
\hline Myristicaceae & 23 & $\begin{array}{l}\text { - Zoochory, including by large- } \\
\text { bodied frugivores } \\
\text { - Hydrochory }\end{array}$ & Yes \\
\hline Ebenaceae & 20 & $\begin{array}{l}\text { - Zoochory, including by large- } \\
\text { bodied frugivores } \\
\text { - Hydrochory }\end{array}$ & Unknown* \\
\hline
\end{tabular}




\begin{tabular}{|l|l|l|l|}
\hline Plant family & $\begin{array}{l}\text { Total No. } \\
\text { of Taxa } \\
\text { within } \\
\text { family in } \\
\text { Moluccan } \\
\text { islands }\end{array}$ & $\begin{array}{l}\text { Dispersal Syndrome(s) among } \\
\text { Moluccan island species }\end{array}$ & $\begin{array}{l}\text { Papuan } \\
\text { hornbill } \\
\text { dispersal w/in } \\
\text { family on } \\
\text { Halmahera? }\end{array}$ \\
\hline Sapindaceae & 19 & $\begin{array}{l}\text { - Zoochory, including by large- } \\
\text { bodied frugivores } \\
\text { Autochory } \\
\text { Anemochory } \\
\text { - Hydrochory }\end{array}$ & Unknown* \\
\hline Sterculiaceae & 19 & $\begin{array}{l}\text { - Zoochory, including by large- } \\
\text { bodied frugivores }\end{array}$ & Unknown* \\
\hline Burseraceae & 17 & $\begin{array}{l}\text { - Zoochory, including by large- } \\
\text { bodied frugivores }\end{array}$ & Yes \\
\hline Rubiaceae & 15 & $\begin{array}{l}\text { - Zoochory } \\
\text { - Autochory }\end{array}$ & Unknown* \\
\hline
\end{tabular}

*There were many seed species found in the Papuan hornbill's excrement that were not possible to identify, and may or may not have been from these families. As these families all contain species with zoochorous dispersal, frugivorous birds such as the hornbill could be involved in their seed dispersal. 
Table 3: Plant species dispersed by the Papuan hornbill including subset from germination trial

(In the habitat occurrences column, 1= primary forest contiguous \& adjacent to protected forest; $2=$ community agroforest in a mixed-habitat landscape; $3=$ primary forest adjacent to unprotected/ mixed use areas. In the germination trial column, $\mathrm{U}=$ species trialled that did not germinate during study period; $\mathrm{Y}=$ species trialled that did germinate.)

\begin{tabular}{|c|c|c|c|c|c|}
\hline Scientific name & Family & $\begin{array}{l}\text { Verna- } \\
\text { cular } \\
\text { name }\end{array}$ & $\begin{array}{l}\text { Seed size } \\
\text { class (in } \\
\mathbf{m m})\end{array}$ & \begin{tabular}{|l|} 
Habitat \\
occurrenc \\
es
\end{tabular} & $\begin{array}{l}\text { Germin- } \\
\text { ation } \\
\text { trial }\end{array}$ \\
\hline $\begin{array}{l}\text { Cananga odorata } \\
\text { (Lam.) Hook.f. \& } \\
\text { Thomson }\end{array}$ & Annonaceae & & $<10$ & 1,2 & \\
\hline Areca vestiaria Giseke & Arecaceae & & $10-19$ & 1,3 & $Y$ \\
\hline $\begin{array}{l}\text { Arenga pinnata } \\
\text { (Wurmb) Merr. }\end{array}$ & Arecaceae & & $20-29$ & $1,2,3$ & $\mathrm{Y}$ \\
\hline $\begin{array}{l}\text { Calamus } \mathrm{L} . \\
\text { sp. A }\end{array}$ & Arecaceae & & $10-19$ & 1 & $Y$ \\
\hline $\begin{array}{l}\text { Calamus L. } \\
\text { sp. B }\end{array}$ & Arecaceae & & $<10$ & 1 & $\mathrm{U}$ \\
\hline $\begin{array}{l}\text { Calamus L. } \\
\text { sp. C }\end{array}$ & Arecaceae & & $10-19$ & 3 & $\mathrm{Y}$ \\
\hline $\begin{array}{l}\text { Caryota rumphiana } \\
\text { Mart. }\end{array}$ & Arecaceae & & $10-19$ & $1,2,3$ & $\mathrm{Y}$ \\
\hline $\begin{array}{l}\text { Pigafetta filaris } \\
\text { (Giseke) Becc. }\end{array}$ & Arecaceae & & $<10$ & $1,2,3$ & $\mathrm{Y}$ \\
\hline $\begin{array}{l}\text { Pinanga rumphiana } \\
\text { (Mart.) J.Dransf. \& } \\
\text { Govaerts }\end{array}$ & Arecaceae & & $<10$ & $1,2,3$ & $\mathrm{Y}$ \\
\hline $\begin{array}{l}\text { Rhopaloblaste } \\
\text { ceramica (Miq.) Burrett }\end{array}$ & Arecaceae & Gelubing & $\begin{array}{l}\text { usually } 20 \\
-29, \\
\text { sometime } \\
\text { s slightly } \\
\text { larger } \\
>30 \mathrm{~mm}\end{array}$ & $1,2,3$ & $\mathrm{Y}$ \\
\hline $\begin{array}{l}\text { Saribus rotundifolius } \\
\text { (Lam.) Blume }\end{array}$ & Arecaceae & $\begin{array}{l}\text { Palem } \\
\text { raja }\end{array}$ & $20-29$ & 1,3 & $Y$ \\
\hline Canarium L. sp. A & Burseraceae & Neara & $20-29$ & $1,2,3$ & $\mathrm{U}$ \\
\hline Canarium L. sp. B & Burseraceae & Kenari & $40-49$ & 1 & $Y$ \\
\hline Canarium L. sp. C & Burseraceae & Kenari & $30-39$ & 1,3 & $\mathrm{U}$ \\
\hline
\end{tabular}




\begin{tabular}{|c|c|c|c|c|c|}
\hline Scientific name & Family & $\begin{array}{l}\text { Verna- } \\
\text { cular } \\
\text { name }\end{array}$ & $\begin{array}{l}\text { Seed size } \\
\text { class (in } \\
\text { mm) }\end{array}$ & $\begin{array}{l}\text { Habitat } \\
\text { occurrenc } \\
\text { es }\end{array}$ & $\begin{array}{l}\text { Germin- } \\
\text { ation } \\
\text { trial }\end{array}$ \\
\hline Trema Lour. sp. & Cannabaceae & & $<10$ & 2 & \\
\hline Carica papaya $\mathrm{L}$. & Caricaceae & & $<10$ & 2 & \\
\hline Calophyllum L. sp. A & Clusiaceae & Hitakono & $30-39$ & 1 & $\mathrm{Y}$ \\
\hline Calophyllum L. sp. B & Clusiaceae & & $20-29$ & 1 & $\mathrm{Y}$ \\
\hline $\begin{array}{l}\text { Calophyllum soulattri } \\
\text { Burm.f. }\end{array}$ & Clusiaceae & & $<10$ & 1,2 & \\
\hline Unknown & Combretaceae & & $10-19$ & 1 & $\mathrm{Y}$ \\
\hline $\begin{array}{l}\text { Cheilocostus speciosus } \\
\text { (J.Koenig) C.D.Specht }\end{array}$ & Costaceae & & $<10$ & 1,2 & \\
\hline Unknown & Cucurbitaceae & & $<10$ & 2 & \\
\hline Eleocarpus L. sp. & Eleocarpaceae & & $10-19$ & 1 & $\mathrm{Y}$ \\
\hline $\begin{array}{l}\text { Macaranga Thouars sp. } \\
\text { A }\end{array}$ & Euphorbiaceae & & $<10$ & 2 & \\
\hline Unknown & Fabaceae & & $<10$ & 1 & \\
\hline Gnetum gnemon L. & Gnetaceae & Ganemo & $10-19$ & $1,2,3$ & $\mathrm{Y}$ \\
\hline $\begin{array}{l}\text { Vitex cofassus Reinw. } \\
\text { ex Blume }\end{array}$ & Lamiaceae & & $<10$ & $1,2,3$ & \\
\hline Aglaia Lour. $s p$. & Meliaceae & & $10-19$ & 2 & $\mathrm{Y}$ \\
\hline Ficus L. sp. A & Moraceae & & $<10$ & 1 & \\
\hline Ficus L. sp. B & Moraceae & & $<10$ & 1 & \\
\hline Ficus L. sp. C & Moraceae & & $<10$ & $1,2,3$ & \\
\hline Ficus L. sp. D & Moraceae & & $<10$ & $1,2,3$ & \\
\hline Ficus L. sp. E & Moraceae & & $<10$ & 1,2 & \\
\hline Ficus L. sp. F & Moraceae & & $<10$ & 3 & \\
\hline $\begin{array}{l}\text { Artocarpus J.R.Forst. \& } \\
\text { G.Forst. sp. }\end{array}$ & Moraceae & & $10-19$ & 2,3 & $\mathrm{Y}$ \\
\hline
\end{tabular}




\begin{tabular}{|c|c|c|c|c|c|}
\hline Scientific name & Family & $\begin{array}{l}\text { Verna- } \\
\text { cular } \\
\text { name }\end{array}$ & $\begin{array}{l}\text { Seed size } \\
\text { class (in } \\
\text { mm) }\end{array}$ & $\begin{array}{l}\text { Habitat } \\
\text { occurrenc } \\
\text { es }\end{array}$ & $\begin{array}{l}\text { Germin- } \\
\text { ation } \\
\text { trial }\end{array}$ \\
\hline Musa L. sp. & Musaceae & $\begin{array}{l}\text { Pisang } \\
\text { hutan }\end{array}$ & $<10$ & $1,2,3$ & \\
\hline $\begin{array}{l}\text { Horsfieldia Willd. } s p . \\
\text { A, }\end{array}$ & Myristicaceae & Dikasuka & $10-19$ & 1 & $\mathrm{U}$ \\
\hline $\begin{array}{l}\text { Horsfieldia Willd. } s p . \\
\mathrm{B} \text {, }\end{array}$ & Myristicaceae & Dikasuka & $10-19$ & $1,2,3$ & $\mathrm{Y}$ \\
\hline $\begin{array}{l}\text { Szygium R.Br. ex } \\
\text { Gaertn. } s p .\end{array}$ & Myrtaceae & & $<10$ & 2,3 & \\
\hline $\begin{array}{l}\text { Glochidion J.R.Forst. \& } \\
\text { G.Forst. } s p .\end{array}$ & Phyllanthaceae & & $<10$ & 2,3 & \\
\hline Unknown & Unknown & Gamunua & $<10$ & 1,3 & \\
\hline Unknown & Unknown & Kori & $10-19$ & 3 & $\mathrm{Y}$ \\
\hline Unknown & Unknown & Mayoro & $10-19$ & 1,3 & $\mathrm{Y}$ \\
\hline Unknown & Unknown & $\begin{array}{l}\text { Lemon- } \\
\text { lemon }\end{array}$ & $10-19$ & 1 & $\mathrm{Y}$ \\
\hline Unknown & Unknown & Ho-oro & $20-29$ & 3 & $Y$ \\
\hline Unknown & Unknown & $\begin{array}{l}\text { Oo-hele, } \\
\text { Kayu raja }\end{array}$ & $>50$ & 1,3 & $Y$ \\
\hline Unknown & Unknown & Kowehe & $10-19$ & 3 & $\mathrm{Y}$ \\
\hline Unknown & Unknown & Hararoko & $10-19$ & 1,3 & $\mathrm{Y}$ \\
\hline Unknown & Unknown & Haya & $<10$ & 1,3 & \\
\hline Unknown & Unknown & $\begin{array}{l}\text { Ngreyeh } \\
\text { oka }\end{array}$ & $10-19$ & 1 & $\mathrm{Y}$ \\
\hline Unknown & Unknown & $\begin{array}{l}\text { Bijuang } \\
\text { gomini }\end{array}$ & $10-19$ & 1 & $Y$ \\
\hline Unknown & Unknown & $\begin{array}{l}\text { Mayoro } \\
\text { daun } \\
\text { halus }\end{array}$ & $<10$ & 1 & \\
\hline Unknown & Verbenaceae & & $<10$ & 1,2 & \\
\hline Leea L. sp. & Vitaceae & & $<10$ & 2 & \\
\hline
\end{tabular}


CHAPTER III

PALM (ARECACEAE) SEED DISPERSAL BY THE PAPUAN

HORNBILL IN WALLACEA 


\section{ABSTRACT}

Dispersal of seeds by birds and other dispersers impacts at least $80 \%$ of woody plant species in the tropics. Large-fruited plant species, especially those that contain a single large seed or few seeds, are likely to be dependent upon the dispersal services of large frugivores, including hornbills (Aves: Bucerotiformes). The palm family (Arecaceae) contains mostly single-seeded drupaceous fruits, and more than half of its species coincide with hornbill habitat in the Old World tropics. To date, little is known about hornbills' role in dispersing palm seeds. Information on the fate of hornbill-dispersed seeds in habitats outside of roost and nest sites is also lacking. Therefore, the aim of this study was to explore dispersal of palms by Papuan hornbills in depth by examining experimentally the fates of hornbill-dispersed palm seeds and their early life history stages in different habitats. An ex-situ shade house germination experiment demonstrated that palm seeds ingested by hornbills experience enhanced germination compared with control seeds. An in-situ germination experiment found that palm seeds ingested by hornbills have a higher rate of germination in four different habitat types and seedling recruitment was higher after one year than in control seeds, although overall recruitment varied between palm species and habitats. One notable conclusion from this study is that dispersal of seeds by hornbills into disturbed habitats results in seedling recruitment, hence hornbills aid both in forest conservation and recovery/assembly after disturbance. Together these results demonstrate that hornbills play a critical ecological role in seed 
dispersal on Halmahera, and their conservation will benefit current and future forests in the Moluccan islands of Wallacea and beyond.

\section{INTRODUCTION}

Seed dispersal and its effectiveness ("SDE") have been widely studied in the Old World tropics, yet gaps in knowledge remain (Schupp et al. 2010). The movement ecology of seeds plays a major role in shaping the composition and long-term persistence of plant communities, including rainforest ecosystems such as those that dominate the Moluccan islands of Indonesia. Dispersal of seeds by birds and other dispersers impacts at least $80 \%$ of woody plant species (Howe and Smallwood 1982, Tiffney 2004, Dennis et al. 2007). Large-fruited plant species (especially those that contain a single large seed or few seeds) are likely to be dependent upon the dispersal services of large frugivores, including hornbills (Wheelwright 1985, Howe 1984 and 1986, Wotton and Kelly 2011, Mueller et al. 2014).

Ranging throughout the Old World tropics, hornbills (Aves: Bucerotiformes) are largebodied frugivorous birds that are among the most important seed dispersers (Kemp 1995, Kinnaird and O’Brien 2007, Kitamura 2011). Hornbills regurgitate or defecate seeds intact with no damage to the seeds, almost always after carrying them away from the parent plant (Kinnaird and O’Brien 2007). Seed dispersal by hornbills is especially important for plants with large capsular fruits such as those in the Meliaceae and Myristicaceae families (Leighton 1986, Corlett 1998, Kitamura 2011), as well as for large 
single-seeded drupes (Kitamura 2011). The palm family (Arecaceae) comprising nearly 2,570 species across 183 genera (Govaerts et al. 2016) has mostly drupaceous fruits, with at least $64 \%$ of its species occurring in the Old World tropics coinciding with hornbill habitat. In a review of zoochorous seed dispersal in palms, Zona and Henderson (1989) found that most palms are dispersed by many different animals, and identified several examples of evidence for long-distance dispersal by frugivorous birds. To date only about 17 species of palms have been documented as consumed by hornbills globally (across both Asia and Africa) (Kitamura 2011, Lamperti et al. 2014, Zona and Henderson 1989).

Hornbills are considered to be agents of effective seed dispersal (e.g., Holbrook 2002, Kinnaird and O'Brien 2007), although a broad review of current hornbill literature suggests that few studies have examined the potential viability of seeds defecated or regurgitated by hornbills (Kitamura 2011). However, across the hornbill species where this has been examined, evidence indicates that seeds defecated or regurgitated by hornbills are able to germinate and in some cases exhibit enhanced germination compared with control seeds (e.g., Whitney et al. 1998, Datta 2001, Balasubramanian and Maheswaran 2003, Kitamura et al. 2008, Santhoshkumar and Balasubramanian 2011). Only a handful of studies have focused upon seed fate beyond initial germination in order to quantify dynamics of seedling survival and recruitment, and most of these studies have focused upon survival around hornbill nest or roost sites (e.g., Kinnaird 1998, Kinnaird and O'Brien 2007, Kitamura et al. 2004, Kitamura et al. 2008, Velho et al. 2009). 
My study seeks to contribute to our understanding of the SDE of hornbills in Wallacea by examining the seeds dispersed by the Papuan hornbill, Rhyticeros plicatus ruficollis, in lowland tropical rainforests of Indonesia and determining its efficacy as a disperser for three palm (Arecaceae) species. Specifically, I asked the following questions: (1) how many and which palm species were ingested by the Papuan hornbills? (2) do palm seeds ingested by the hornbill have a higher germination rate than palm fruits and/or seeds not ingested by the hornbill? (3) what happens to palm seeds ingested by hornbills after they are deposited in four representative habitats on the island (i.e., primary forest, conspecific "parent" palms, roosting sites, and disturbed areas)? For the last two questions I examined three palm species not only because palms are a prominent group of plants in the island's natural vegetation that can be recognized with relative ease, but also because palms have not yet been widely studied in terms of potential dispersal by hornbills.

\section{MATERIALS AND METHODS}

\section{Study site}

This study was conducted on the Indonesian island of Halmahera, the largest island in the Moluccas, with Sulawesi to the west, Papua to the east, and the Philippine island of Mindanao to the north. Halmahera covers an area of approximately $17,780 \mathrm{~km}^{2}$ and is home to the province's only National Park ("NP"), which was established in 2004 to encompass approximately 167,300 hectares that are split into two disjunct NP blocks, the Aketajawe block and the Lolobata block. Ecologically the island of Halmahera is a 
priority conservation area: it contains several Important Bird Areas ("IBAs") (BirdLife International 2016) and occurs in the Wallacea Biodiversity Hotspot (Myers et al. 2000). Prior to this study, there were not any recent studies on plant-animal interactions on the island.

The study was carried out at several sites on Halmahera island (Figure 1), categorized herein as either "seed collection sites" or "in-situ experimental sites," with both categories including sites adjacent to Aketajawe-Lolobata National Park in a contiguous forest matrix. The seed collection study sites were all located within $0-10 \mathrm{~km}$ distance to primary moist evergreen tropical forest and were selected on the basis of the following criteria: 1) accessibility of hornbill roost tree, 2) ensuring each of three seed collection habitat types had a "representative" roost tree, and 3) the roost tree was occupied by hornbills for the majority of the study duration. The seed collection habitat types were: 1) primary forest contiguous with a protected forest, 2) primary forest adjacent to mixed land uses, and 3) community agroforest adjacent to mixed land uses and disturbed areas. The in-situ experimental study of palm seed fates was conducted in four different habitat types that are representative of habitats that are common across Halmahera as well as other islands in Wallacea, defined here as: 1) "parent" palm, 2) hornbill roost tree, 3) primary forest, with potential perch trees present and 4) disturbed area lacking closed canopy, but with remaining perch/food trees present.

The island of Halmahera receives a relatively high yet varied amount of precipitation, with mean annual rainfall of approximately $2000 \mathrm{~mm}$ in the southern part of the island up 
to about $4000 \mathrm{~mm}$ in the northwest part of Halmahera (Suparan et al. 2001). While rain is common throughout the year, the slightly dry months tend to be July, August, and September.

Study species

The Papuan (or Blyth's) hornbill, Rhyticeros plicatus ruficollis, is the only hornbill species native to Halmahera. It is one of six subspecies of the $R$. plicatus species complex (Clements et al. 2016). It is a large frugivorous bird that is relatively common, although declining, throughout the island of Halmahera (M. Abdo, unpublished data). Papuan hornbills on Halmahera are found both in and adjacent to primary and secondary forests, in "ecotones" and disturbed areas between agroforests, in fallow lands, and in alluvial zones (M. Abdo, unpublished data).

The remaining focal species of this study are the palms (Arecaceae) of Halmahera island. Halmahera has a diverse flora, and the palm flora including all native and naturalized palms extant in Halmahera is estimated to include 22 native genera (Govaerts et al. 2016, and Chapter 1 of this dissertation). The "botanical country" in which Halmahera island occurs is the Moluccan islands (including the Indonesian provinces of North Maluku and Maluku), a subregion of the Wallacea biogeographical region and hotspot. An estimated 47 native palm taxa are present in the Moluccan islands (Govaerts et al. 2016, and Chapter 1 of this dissertation). 
Three particular species of palm were examined in the comparative germination experiments of this study to determine the efficacy of hornbill seed dispersal. Pigafetta filaris (Giseke) Becc., is a dioecious unbranched polycarpic palm that may grow up to 50 meters in height, and has been called a "strict heliophile" during its juvenile stage, when it is not known to grow past a seedling unless it is receiving plenty of open sunlight (Dransfield 1976). Its leaflets are up to about $1 \mathrm{~m}$ long on pinnate leaves that are up to about $6 \mathrm{~m}$ long, with petioles about $1.8 \mathrm{~m}$ long and having a whitish powder covering at their bases. Pigafetta filaris has axillary inflorescences with pendulous branches, all cream-yellow in color, reaching up to about $2 \mathrm{~m}$ long and bearing fruits that are about $12 \mathrm{~mm}$ in length, ovoid, single-seeded, and covered by imbricate yellow to orange colored scales (Dransfield et al. 2008). Rhopaloblaste ceramica (Miq.) Burret is a monoecious unbranched polycarpic palm that may grow up to an estimated 38 meters in height, and is the tallest species in its genus. Its slender leaflets grow up to about $1 \mathrm{~m}$ long on pinnate leaves that are up to about $4 \mathrm{~m}$ long, with the leaf sheath and petiole being about $2 \mathrm{~m}$ long. Rhopaloblaste ceramica has inflorescences that are branched to three orders that reach $1.5 \mathrm{~m}$ long and across, bearing single-seeded ellipsoid fruits that are typically $30-35 \mathrm{~mm}$ long, yellow ripening to red in color (Dransfield et al. 2008). Saribus rotundifolius (Lam.) Blume is a hermaphroditic unbranched polycarpic palm that typically fruits and flowers all year, and grows up to $50 \mathrm{~m}$ in height. It has costapalmate leaves up to $1.5 \mathrm{~m}$ long on petioles up to $2 \mathrm{~m}$ long. Saribus rotundifolius has inflorescences that are branched to four orders that reach about $1.5 \mathrm{~m}$ long bearing globose single-seeded fruits 
usually $20 \mathrm{~mm}$ in diameter, yellow ripening to red or bluish-black in color (Dransfield et al. 2008). Pigafetta filaris and $R$. ceramica have narrow ranges, occurring only in the Moluccan islands and New Guinea, compared to S. rotundifolius which occurs in Malaysia, Borneo and eastern Indonesia, and the Philippines.

\section{Seed collecting, sorting and identification}

Seeds were collected from hornbill- excreted and regurgitated seeds collected in seed traps below roosting sites. Three known hornbill roosting locations were surveyed over 12 months in order to capture hornbill-ingested seed samples. Hornbills themselves were not handled nor their roosting trees disturbed in any way during the course of this study. Roosting locations consisted of tall emergent trees, one in each of the three different seed collecting habitats, and were frequented by at least fifteen hornbills each (on a recurring basis) and occasionally up to forty-six individuals simultaneously. One sampling location was located adjacent to the Aketajawe block of the Aketajawe-Lolobata National Park in east Halmahera in primary forest habitat; one sampling location was within a community agroforest/secondary forest matrix in central Halmahera; and one was located within unprotected primary forest in North Halmahera, adjacent to mixed land uses. Each location was visited a minimum of 4 times during the 12-month period to survey hornbill roosting activity for consecutive 5-day watches. (Note: additional roosting locations were originally chosen for the study, however during the study period hornbills had been extirpated from these sites because of hunting, a relatively new local phenomenon discussed later). 
Sampled collections of hornbill-ingested seeds captured in traps were then sorted. All seeds (across all plant families) were identified at least to the level of genus and/or by vernacular names (see Chapter 1 for details on all the seed species), and all palm seeds were identified to either genus or species level. Measurements of all palm seeds found in hornbill excrement were collected using digital calipers. The palm seeds deposited by hornbills in the seed traps formed part of the basis for the experiments described below.

\section{Ex-situ comparative germination trials}

The ex-situ component of this study was carried out using germination trials in a shade house in order to ascertain the germination rate of hornbill-ingested palm seeds in comparison to palm seeds not ingested by hornbills. The germination experiment was conducted over fourteen weeks and was concluded once no signs of germination (i.e., emergence of a radicle) were observed for two consecutive weeks.

The nursery germination trials were carried out with the three aforementioned palm species (Pigafetta filaris, Rhopaloblaste ceramica, and Saribus rotundifolius), using three treatments: 1) seed that was hornbill-selected, consumed, and passed through the digestive tract of the hornbill i.e., obtained from fresh hornbill excrement or regurgitated material; 2) seed that was human-selected at maturity and had its pulp removed manually; and 3) mature fruits with no manipulations done i.e., pulp/pericarp intact. The seeds from the first treatment (i.e., seeds ingested by the hornbills) were collected from fresh samples taken from three different sampling locations (as outlined above). The seeds from the 
second and third treatments were collected from mature palms, at least five individual palm plants per species. A total of 120 seeds per species per treatment were used in the nursery germination experiment, which consisted of two replications (60 seeds per replication). The experimental setting was an open-sided nursery under approx. $30 \%$ shade cloth. All seeds were planted in plastic pots in a mixture of local-derived leaf and organic matter humus $(\sim 70 \%)$ mixed with peat moss $(\sim 30 \%)$. Humidity readings were consistently over $80 \%$ (due to ambient humidity) and irrigation was applied to ensure the media was kept slightly moist.

In-situ palm seed germination and seedling survival experiment

The in-situ component of this study was conducted in order to ascertain the germination rate and early life seed fate of hornbill ingested palm seeds, in comparison to palm seeds not ingested by hornbills as controls, in a variety of habitats where seed deposition could potentially occur. The four habitat types studied are also representative of habitats common in Halmahera and other Moluccan islands. In some of these habitats, palm seeds that are not ingested and deposited by hornbills (i.e., palm seeds with fruit pulp/ exocarp intact) might be transported/dispersed by other modes such as other frugivores or abiotic dispersal e.g., heavy rains, rolling down slopes, (M.Abdo unpublished data). Distances of dispersal from parent palms by other such vectors are unknown.

This experiment was carried out using the same three palm species (Pigafetta filaris, Rhopaloblaste ceramica, and Saribus rotundifolius) as in the aforementioned shade house 
germination study, with the same three treatments and seed sources as outlined above. However, in these experiments a total of 50 seeds (instead of 60) per species per treatment were used at each of the four different in-situ habitat settings, with two replications done per habitat (a total of twelve experimental sites), equalling a total of 100 seeds for each unique species, treatment, and habitat combination. The total number of seeds used of a given palm species across all treatment and habitat types was 1200 . The four different habitats where the palm seeds were planted were chosen according to realistic possibilities that seed deposition by hornbills may occur in these habitats and because they are representative of habitats common on Halmahera: 1) beneath the "parent" palm; 2) beneath hornbill roosting trees; 3) within primary forest below potential perching trees; and 4) within disturbed sites lacking canopy cover, but with potential perching/feeding trees still present. Active nesting sites were not included in this experiment as Papuan hornbills appeared to be very sensitive to our movements when around nesting trees, with human presence/movement potentially disruptive to male feedings to the nesting female (M. Abdo and Mahroji, unpublished data). On the other hand, our activity around the roosting (vs. nesting) trees did not appear to impact or disturb the hornbills' typical behavior, and we also timed our study visits during the day hours when hornbills were normally not at the roost site so as to avoid any potential disruptions to the hornbills. Two replicates of each habitat type were used simultaneously. At each "parent" palm habitat only corresponding (conspecific) seed species were examined. Two mature palms of each of the three palm species (a total of 
six experimental sites across all palm species) were used, with a total of 150 samespecies seeds utilized experimentally at each parent palm site. Across both replicated sites 300 total seeds were used, with a total of 100 seeds per species and treatment combination in total per replicated parent palm habitat. For the remaining three habitat types (each replicated twice), the three palm seed species and treatments were tested concurrently at the same six sites with a total of 100 seeds for each unique palm species and treatment combination per habitat type.

Palm seed species were easy to distinguish from one another. However, to aid in discerning the different treatment types within sets of species two simple methods were used. First, seeds of a unique treatment type were marked in different colors with permanent paint marker to aid in determining if planted seeds rolled (or were moved around by biotic/abiotic factors) after the initial planting stage. Second, grouped planting areas under the crowns of each experimental site/tree were clearly diagrammed (see Figure 2). "Planting" of the various treatments of palm seeds is defined in this experiment as placing the seeds firmly on the substrate that was present at each site, embedding them into the substrate approximately $0-25 \%$ of their seed diameter. Substrates were not otherwise disturbed and no surrounding seedlings, leaves/humus, or grass (in the case of the disturbed habitats) was removed. At each site, half of the seeds were planted close to the center of the tree's crown, i.e., $0.5-2.5 \mathrm{~m}$ from the trunk, and the other half was placed further out from the middle to edge of the crown i.e., $2.5 \mathrm{~m}-6 \mathrm{~m}$ from the trunk (Figure 2). 
Experimental sites were observed frequently (typically two sites each day) over the course of the first four weeks post-planting to collect observations on planted seeds. I specifically observed for signs of germination (defined here as a radicle and/or plumule emerging), signs of seedling growth/recruitment (i.e., establishment past germination stage), signs of predation damage (e.g., bore holes made by seed-predating insects or bite marks of small scavengers), disappearance (possibly via transport away from site by a terrestrial scavenger, or rolling away by abiotic factors), or pathogens (e.g., rot and/or apparent disintegration of seed). Subsequent to the first four weeks post-planting, all sites were visited at week ten and twelve, followed by week sixteen, twenty, and forty-six post-planting. This observation schedule was aligned purposefully with the experiment in that the majority of the palm seeds germinated fairly rapidly, varying from one to ten weeks post-planting across all three palm species.

\section{Statistical analyses}

In order to analyze the results of the in-situ palm seed germination experiment, a twoway analysis of variance (ANOVA) with a significance of 5\% was used. The cumulative number of seeds that germinated or recruited as seedlings was considered as the measurement variable. The seed treatment (i.e., hornbill ingested, manual pulp removal, and intact fruits) was the first nominal/categorical variable and the habitat was the second nominal variable. In order to obtain insightful statistics while examining multiple variables in this in-situ experimental setting, each palm species was analyzed separately (unlike the ex-situ setting wherein palm species were pooled into the same analysis, as 
described below). Post-hoc Tukey Honestly Significant Difference (Tukey HSD) tests were also conducted. Analyses were carried out at four different stages in the experiment. First, germination was analyzed after the majority of seeds had germinated (this occurred by the tenth week in all palm species planted in in-situ settings). Second, seedling recruitment was analyzed at the sixteen-week point, followed by seedling survival at the twentieth week and finally at the forty-sixth week. The two-way ANOVA is appropriate to test this particular experiment with one measurement variable and two nominal or categorical variables, and the post-hoc Tukey HSD test provides additional clarity on the results of the two-way ANOVA (McDonald 2014).

In order to analyze the results of the shade house (ex-situ) experiment, each of the palm species with its unique treatment was analyzed to result in nine total possible combinations of palm species (of which there were three) and treatments (of which there were three). Emergence of a radicle indicated germination, and any result otherwise indicated lack thereof. Analysis was carried out using a two-way ANOVA test (McDonald 2014) to determine if there was a significant difference in the means of seed species that germinated or the different palm seed treatments, with a significance of $5 \%$. A post-hoc Tukey HSD test was then used to examine results between all pairs of treatments in further detail. 


\section{RESULTS}

\section{Palm seed dispersal by hornbills in Halmahera}

Examination of the seeds ingested by Rhyticeros plicatus ruficollis yielded at least 71 distinct plant taxa (see Chapter 2 of this dissertation) and 10 distinct palm seed species identified in the hornbills' excrement/regurgitated material (see Table 1) in total from 3 locations on the island of Halmahera. The primary forest habitat site contiguous with the Aketajawe block of the National Park had 8 palm species, followed by the primary forest contiguous with unprotected habitat and mixed-use areas having 7 palm species, and the community agroforest/secondary forest site having seeds from 5 palm species. The fruit sizes of these palms varied from less than $10 \mathrm{~mm}$ to over $30 \mathrm{~mm}$ in diameter. Their colors at maturity also vary from dark greenish/brownish to orange to red to purple and bluishblackish. I was able to identify all the palm seed species except for three Calamus spp. that were identified to the level of genus.

\section{Ex-situ germination experiment}

The nursery germination trials of the three palm species ingested by the hornbill demonstrated that the hornbill provides a high-quality dispersal service for Pigafetta filaris, Rhopaloblaste ceramica, and Saribus rotundifolius. Across all three (pooled) palm species, the mean number of seeds that germinated in each trial $(n=60)$ that were ingested by hornbills, had their pulp removed manually, or were intact fruits with no manipulations done were $47.50( \pm 0.866), 47( \pm 7.000)$, and $10.83( \pm 4.856)$ respectively 
(Figure 3). The ANOVA analysis indicated that there was not a significant effect on the results caused by the species of palm seed. A significant effect was observed in the seed treatments (ANOVA; $\left.\mathrm{F}_{2,9}=121.19, \mathrm{P}<.0001\right)$, which accounted for $91.54 \%$ of the total variance. A post-hoc Tukey HSD test indicated that there was not a significant difference between the germination rates of different palm species that underwent the same seed treatment. There were no significant differences between the germination rate of any species of the hornbill-ingested seeds and any species of seeds that had their pulp removed manually. Across all palm species, the intact fruit (control) treatment had a significant negative effect on germination in comparison to the other two treatments (Tukey, in all tested comparisons $\mathrm{P}<.001$ ).

\section{Pigafetta filaris}

The mean number of Pigafetta filaris seeds that germinated that were ingested by hornbills, had their fruit pulp removed manually, and controls with fruit/pulp intact were $48( \pm 8.485), 52( \pm 4.243)$, and $15( \pm 5.657)$, respectively. Tukey HSD analysis revealed that there was not a significant difference between the two $P$. filaris treatments that had their pulp removed (by the hornbill and by manual removal), but there were significant differences between both of those treatments in comparison to the intact fruit control treatment (Tukey, $\mathrm{P}<.001$ in both cases). 


\section{Rhopaloblaste ceramica}

The mean number of Rhopaloblaste ceramica seeds that germinated that were ingested by hornbills, had their fruit pulp removed manually, and controls with fruit/pulp intact were $46.5( \pm 4.950), 50( \pm 4.243)$, and $5.5( \pm 2.121)$, respectively. Tukey HSD analysis revealed that there was not a significant difference between the two $R$. ceramica treatments that had their pulp removed (by the hornbill and by manual removal), but there were significant differences between both of those treatments in comparison to the intact fruit control treatment (Tukey, $\mathrm{P}<.0001$ in both cases).

\section{Saribus rotundifolius}

The mean number of Saribus rotundifolius seeds that germinated that were ingested by hornbills, had their fruit pulp removed manually, and controls with fruit/pulp intact were 48 ( \pm 4.243$), 39( \pm 1.414)$, and $12( \pm 2.828)$, respectively. Tukey HSD analysis revealed that there was not a significant difference between the two $S$. rotundifolius treatments that had their pulp removed (by the hornbill and by manual removal), but there were significant differences between both of those treatments in comparison to the intact fruit control treatment (Tukey, $P<.001$ in both cases). 
$\underline{\text { In-situ germination and seedling recruitment of hornbill-dispersed palms }}$

Pigafetta filaris

Germination of the Pigafetta filaris seeds was influenced the most by the seed treatments, which accounted for approximately $82.02 \%$ of the total variance in effect (Fig. 4). Habitat types also had significant effect on germination (ANOVA; $\mathrm{F}_{3,12}=7.33, \mathrm{P}<.01$ ), accounting for about $7.56 \%$ of the total variance (Fig. 4). The intact palm fruits (used as controls, mimicking autochoric or abiotic dispersal) had significantly lower germination, across all habitat types, in comparison to the other two treatments of palm fruits (ANOVA; $\mathrm{F}_{2,12}=119.25, \mathrm{P}<.0001$ ). Tukey analysis revealed that there was not a significant difference between germination of the hornbill ingested or pulp-removed seeds, at any habitat site.

Interestingly, successful recruitment of Pigafetta filaris seedlings at the end of the study was influenced by both the habitat and seed treatment (Fig. 5). The seed treatments accounted for approximately $41.44 \%$ of the total variance in effect $\left(\right.$ ANOVA; $F_{2,12}=$ 18.22, $\mathrm{P}<.001)$. Habitat types also had significant effect on recruitment $\left(\right.$ ANOVA; $\mathrm{F}_{3,12}=$ $10.04, \mathrm{P}<.01$ ), accounting for about $34.24 \%$ of the total variance. Within primary forest and parent palm habitats, seed treatment did not have a significant effect on recruitment success. At the roost site, only hornbill ingested seeds experienced a significant positive effect in their survival in comparison to the control seeds (Tukey, $\mathrm{P}<.01$ ). Within disturbed habitats, both the hornbill ingested and pulp-removed seeds resulted in 
significantly higher recruitment (Tukey, $\mathrm{P}<.01$ and $\mathrm{P}<.05$, respectively) in comparison to control seeds.

Further examination of interactions between variables revealed that across the total set of control seeds there was no significant difference between recruitment at different habitat types. However, across the hornbill ingested seed set, recruitment was significantly higher at roost sites compared with primary forests (Tukey, $\mathrm{P}<.05$ ) and significantly lower in primary forests compared to disturbed sites (Tukey, $\mathrm{P}<.05$ ). Seeds that had their pulp removed manually demonstrated significantly lower recruitment at both parent palm habitats and primary forest habitats compared to disturbed sites (Tukey, $\mathrm{P}<.05$ in both cases).

\section{Rhopaloblaste ceramica}

Germination of the Rhopaloblaste ceramica seeds was influenced the most by the seed treatments, which accounted for approximately $71.48 \%$ of the total variance in effect (Fig. 6). Habitat types also had significant effect on germination (ANOVA; $\mathrm{F}_{3,12}=7.83$, $\mathrm{P}<.01$ ), accounting for about $14.14 \%$ of the total variance (Fig. 6). The intact (i.e., control) palm fruits had significantly lower germination, across all habitat types, in comparison to the other two treatments of palm fruits (ANOVA; $\mathrm{F}_{2,12}=59.38, \mathrm{P}<.0001$ ). Tukey analysis revealed that there was not a significant difference between germination of the hornbill ingested or pulp-removed seeds, at any habitat site. 
The interaction between the seed treatment and habitat type that Rhopaloblaste ceramica seeds were planted within was statistically significant to overall recruitment at the end of the study period, accounting for $23.23 \%$ of the overall variance (ANOVA; $\mathrm{F}_{6,12}=9.07, \mathrm{P}$ $<.001$ ) (Fig. 7). The seed treatments accounted for approximately $28.33 \%$ of the total variance in effect (ANOVA; $\left.\mathrm{F}_{2,12}=33.18, \mathrm{P}<.0001\right)$, while habitat types also had a significant effect on recruitment (ANOVA; $\mathrm{F}_{3,12}=33.82, \mathrm{P}<.0001$ ), accounting for about $43.31 \%$ of the total variance.

Further analysis using Tukey's multiple comparisons test revealed that across most habitats there was no significant difference in the overall survival of seedlings from seeds that had their pulp removed either via hornbill ingestion or manually, except for at the primary forest sites. Within primary forest habitat, hornbill ingested seeds had somewhat higher seedling survival than seeds with their pulp removed by hand (Tukey, $\mathrm{P}<.01$ ) and both types of seed treatments mimicking frugivore dispersal (hornbill ingestion and manual pulp removal) fared better than control seeds (Tukey, $\mathrm{P}<.0001$ and $\mathrm{P}<.001$ respectively). In contrast, the parent palm and disturbed habitats had less variability in overall seedling survival. All seed treatments fared poorly (no significant differences) over time at the parent palm sites. Within disturbed habitats, only the seeds with their pulp manually removed resulted in slightly higher seedling survival than control seeds (Tukey, $\mathrm{P}<.05$ ). Within the roost habitat, the hornbill ingested seeds also resulted in slightly higher seedling survival than control seeds (Tukey, $\mathrm{P}<.05$ ). 
Further examination of interactions between variables revealed that across the total set of control seeds there was no significant difference between recruitment at different habitat types. However, across the hornbill ingested seed set, recruitment was significantly lower at every non-primary forest habitat (i.e., roost, parent palm, and disturbed sites) when compared with recruitment at the primary forest habitat site (Tukey, $\mathrm{P}<.0001$ in all cases). Seeds that had their pulp removed manually demonstrated significantly higher recruitment in primary forest habitats as well, compared with roost, parent palm, and disturbed habitats (Tukey, $\mathrm{P}<.01, \mathrm{P}<.01$, and $\mathrm{P}<.05$ respectively).

\section{Saribus rotundifolius}

Germination of the Saribus rotundifolius seeds was influenced strongly by both the seed treatments as well as the type of habitat the seeds were planted in. The seed treatments accounted for approximately $47.57 \%$ of the total variance in effect, while habitat types accounted for approximately $34.03 \%$ of the total variance in effect (Fig. 8). The intact palm fruits (i.e., controls) had significantly lower germination, across all habitat types, in comparison to the other two treatments of palm fruits (ANOVA; $\mathrm{F}_{2,12}=149.23, \mathrm{P}<.0001$ ). Tukey analysis revealed that there was not a significant difference between germination of the hornbill ingested or pulp-removed seeds, at any habitat site. Further Tukey analysis of the interaction between variables revealed that only at the disturbed habitat site there was not a significant difference in germination for any Saribus rotundifolius 
seeds that underwent different seed treatments (germination was poor across the board at the disturbed habitats).

At the end of the study, successful recruitment of Saribus rotundifolius seedlings was influenced by both the habitat and seed treatment (Fig. 9). The interaction between the seed treatment and habitat type that Saribus rotundifolius seeds were planted within was not quite significant $(\mathrm{P}<.1)$ although it accounted for $18.79 \%$ of the total variance. The seed treatments accounted for approximately $30.06 \%$ of the total variance in effect $\left(\right.$ ANOVA; $\left.\mathrm{F}_{2,12}=11.70, \mathrm{P}<.001\right)$. Habitat types also had significant effect on recruitment (ANOVA; $\mathrm{F}_{3,12}=9.27, \mathrm{P}<.001$ ), accounting for about $35.74 \%$ of the total variance.

However, within parent palm and disturbed habitats, there was not a significant difference in the overall recruitment of palm seedlings between any seed treatments. In contrast, at the roost and primary forest sites, hornbill ingested seeds and those with their pulp removed manually experienced similar significant positive effects in their survival in comparison to the control seeds (Tukey, $\mathrm{P}<.05$ and $\mathrm{P}<.01$, respectively).

Further examination of interactions between variables revealed that across the total set of control seeds there was no significant difference between recruitment at different habitat types. However, across the hornbill ingested seed set, recruitment was significantly higher at primary forests compared with parent palm and disturbed habitats (Tukey, $\mathrm{P}<$. 05 and $\mathrm{P}<.01$ respectively). Seeds that had their pulp removed manually also demonstrated significantly higher recruitment at primary forests compared with parent 
palm and disturbed habitats (Tukey, $\mathrm{P}<.05$ and $\mathrm{P}<.01$ respectively), and additionally at roost habitats compared with disturbed sites (Tukey, $\mathrm{P}<.05$ ).

\section{Additional observations}

While this study does not focus on the reproductive or movement behavior of Papuan hornbills, we report that hornbills in Halmahera tend to roost in emergent or high-canopy trees (including healthy trees with intact canopies and defoliated or dead trees) in both primary forest and mixed-habitat areas including zones in the periphery of human-used agricultural areas. In contrast, Papuan hornbill nesting behavior was not observed in any trees adjacent to human-used or heavily degraded areas but rather only in primary forests or minimally disturbed forests.

One roost site I studied was located in close proximity to a small river and hornbilldigested seeds often fell into the river and while many viable seeds sank, some viable seeds were carried away by the current (frequent changes in the water levels could easily deposit them ashore). This is an example of how seeds from roost sites can also experience secondary abiotic dispersal.

\section{DISCUSSION}

Seed dispersal service by hornbills in Halmahera

I found that the Papuan hornbill ingests and disperses at least 10 different species of palms in Halmahera. The nursery-based experiment demonstrated that hornbills have a 
positive effect in enhancing germination rates of palm seeds in comparison to that of palm fruits that had no pulp removed (when environmental factors were otherwise the same across species and treatments). This indicates that Papuan hornbills, and possibly other large-bodied frugivores that are capable of removing the pulp of diaspores prior to seed deposition/dispersal, play an important role in SDE of the palm species they disperse. There was no significant difference in germination between hornbill-digested seeds and seeds that had their pulp removed manually in the ex-situ experiment. On the basis of observations made during the study, the mechanism for why seeds with pulp removed (either by hornbills or manually) fared better than control seeds is unclear, but possible explanations could be that intact fruit seemed to attract both fruit/seed predators (e.g., boring insects) as well as facilitate rot/fungal growth and both of these factors contributed at least in part to the demise of the control seeds that did not germinate.

An important finding of this study is that without frugivore consumption/ingestion resulting in fruit pulp removal, all three palm species experienced significantly lowered or zero germination and recruitment; this result could very well represent the case for other palm species and the numerous drupaceous plant species in the Moluccan islands. Further, this finding validates increasing concerns over the impacts of frugivore declines on plant communities (Vanthomme et al. 2010, Wotton and Kelly 2011, Howe 2016). Dispersal by hornbills imparted benefits to palms in a range of habitats in Halmahera (Fig. 10), in marked contrast to control palm seeds. The results of the experimental field study indicate that some hornbill-dispersed palm seeds do survive to recruit into seedlings 
almost a year post-dispersal in several different habitats. This is an important contribution as only a couple of studies have investigated hornbill-dispersed seed fates in habitats other than roosting or nesting sites (i.e., Kitamura et al. 2008, Datta 2001), and understanding how dispersal by hornbills (and other large-bodied frugivores) acts as a mechanism contributing to plant community assembly in a variety of habitats is desirable.

In primary forests, palm seeds undergoing pulp removal (either manually or by hornbill ingestion) successfully recruited and survived almost a year post-dispersal, with recruitment success above $29 \%$ and $42 \%$ respectively for two of the tested palm species, Rhopaloblaste ceramica and Saribus rotundifolius and at about 4.5\% for Pigafetta filaris. However, in disturbed habitats, Pigafetta filaris seeds undergoing pulp removal (either manually or by hornbill ingestion) experienced about $29 \%$ survival at the close of the study, while Rhopaloblaste ceramica and Saribus rotundifolius seedlings only had about $8.5 \%$ and $1.5 \%$ recruitment success (respectively) after nearly a year. As presented earlier in the results, recruitment also varied in the roost and parent palm sites. The significant variability in recruitment success of different palm species in different habitats could have been caused by differing abiotic resource requirements of the palm species during their early life stages (e.g., precipitation and ambient humidity, light, soil nutrients) and the varied availability of such resources in the different habitats studied. Biotic factors such as herbivory, rhizospheric interactions, and the influence of pathogens could also play roles in the varied recruitment success. Temporal/seasonal variations and stochastic events may also impact the early lives of plants. Indeed, the aforementioned 
wealth of factors may have played a part in the increased proportion of variance attributed to "influence of habitat" observed a year post-dispersal compared with postgermination in two of the species, $R$. ceramica and P. filaris.

Howe (2016) has highlighted that understanding seed dispersal dynamics in disturbed habitats adjacent to forests is increasingly important given the declining state of tropical landscapes today, and this study's finding that seeds undergoing dispersal treatment (i.e., pulp removal and movement away from the parent plant) have increased survival in disturbed habitats is noteworthy. Observations made during this study of hornbills present and/or perching in all the habitats studied indicates that seed deposition into a range of habitats (including disturbed areas) is feasible in Halmahera. Therefore, frugivores such as Papuan hornbills contribute not only to plant community assembly in primary forests, but also to regeneration and assembly processes in disturbed habitats as they aid in moving plant diaspores into disturbed sites. Frugivorous birds and bats have been shown to aid in rebuilding island plant communities elsewhere in Indonesia (Whittaker and Jones 1994).

Several factors contribute to the overall SDE of Papuan hornbills as dispersal vectors. Hornbills have the capability of ingesting a relatively large quantity of palm fruits during their feeding visits (Chapter 2 of this dissertation). Furthermore, Papuan hornbills have the potential for long-distance dispersal as they are able to fly at least $10-15 \mathrm{~km}$ in a day (Kinnaird \& O'Brien 2007) and in Halmahera may travel more than $11 \mathrm{~km}$ in a day (see Chapter 2 of this dissertation). Hornbills traveling such distances while carrying 
diaspores helps plant species to colonize a range of habitats, and to avoid densitydependent mortality around parent plants (Jansen 1970, Howe 1984) such as was evidenced at the conspecific habitat sites in this study.

In summary, the combined high-quality and high-quantity dispersal activity, being key components of evaluating SDE (Schupp et al. 2010), demonstrate that Papuan hornbills are indeed effective seed dispersers for palms in Halmahera. Future study to follow the fate of surviving seedlings in order to learn more about their long-term survival is ultimately an important and under-examined aspect of SDE (Schupp et al. 2010, Traveset et al. 2014) and hornbill SDE in particular (Kitamura 2011). This study illustrates that hornbills impact the SDE of palms in Wallacea by enhancing their recruitment into primary forests, roost sites, and into disturbed landscapes where forest regeneration is desirable.

\section{Ecological notes on hornbill-dispersed species of Halmahera island}

One of the 10 palms dispersed by the Papuan hornbill, Pigafetta filaris, requires significant sunlight in order to grow into a healthy palm (Dransfield 1978), and where the Papuan hornbill is extant, P. filaris has been observed to often be one of the first tree/ palm species to colonize open areas disturbed by humans or natural causes (e.g., treefalls, landslides) (M. Abdo, pers. obs.). Among the other palms found to be dispersed by Papuan hornbills, Arenga pinnata and Caryota rumphiana are also known to be earlysuccession species. It is likely that hornbill seed deposition of these early colonizers has 
contributed to re-establishing plant growth into disturbed areas. Furthermore, if we consider the large species pool of potential plant colonists into such disturbed areas, it is unlikely that medium- and large- sized fruit/plant species would be dispersed into disturbed areas without the dispersal services of large birds such as hornbills. Many of these medium- and large- sized fruit species in Halmahera consist of tall trees and palms (M. Abdo, unpublished data), including trees such as Ficus spp. that are important sources of food/shelter for many animals. Pigafetta filaris is a rapidly growing palm tree, and in about a decade stands of the palm can grow in previously disturbed areas to provide shade or microhabitats for less tolerant plant species. Importantly, P. filaris can also provide perching habitat (that early-colonizing smaller shrubs/grasses cannot provide) for bird and bat species with varied diets that then may contribute to further seed dispersal into the community. The dispersal service of hornbills may very well be an important mechanism by which local plant communities assemble after disturbance.

Areca vestiaria and Pinanga rumphiana typically grow to be low- to mid-story palms in Halmaheran forests, and are relatively common members of the island's understory forest plant communities, especially in alluvial and karst plant communities in Halmahera's forests (M. Abdo, unpublished data). Both of these species provide important food and nectar sources for other wildlife (e.g., birds, bats, butterflies, and insects) (M.Abdo, pers. obs.). Calamus spp., climbing rattan palms, are common and frequently abundant components of forest communities and play varying roles in the plant community structure, from creating rattan-dominated understory habitats to rattan-sparse 
communities where they climb and are eventually emergent palms. Several endemic and rare birds are closely associated with the rattan-dominant forest communities. Last but not least, the tall and often-emergent Rhopaloblaste ceramica and Saribus rotundifolius palms are also relatively common in Halmaheran forests, the latter species more frequently encountered on hill ridges than in lower elevations in Halmahera. However, dense stands of these two species of palms have not been found in Halmahera (unlike with Pigafetta filaris), and in some areas subpopulations of these two palm species appear to be limited to specific microhabitats. Both species provide important food sources for wildlife, especially bats and birds. All of these palm species can be longlived.

Outside of the palm species that the Papuan hornbill disperses, there are many important tree species that the hornbill disperses, including both short-lived early successional species (e.g., Macaranga sp., Trema sp., Musa sp.) as well as many long-lived tree species (see Chapter 2 of this dissertation). Several of the species it disperses have relatively large seeds, such as Canarium spp. with seeds $\sim 30$ - 40mm in length, and the unidentified tree species "oo-hele" with giant $\sim 52 \mathrm{~mm}$ oblong seeds. Papuan hornbills appear to consume and disperse a variety of plant species whose functional dispersal traits vary. While we do not know why hornbills choose to consume both large and small fruits, we did learn that in Halmahera they are not limited to consuming the classic birddispersal syndrome range of yellow-to-red-to-purple fruits but they also consume fruits that are green and brown (colors often associated with bat dispersal) as well as some 
fruits that are not very fleshy and have rather hard exocarps (e.g., Pigafetta filaris, Calamus spp.). Other studies have shown that hornbills track fruit resources

(Naniwadekar et al. 2015b) and selectively consume different fruits according to their breeding season (Lamperti et al. 2014). Papuan hornbills are indeed keystone dispersers not only in terms of their SDE, but also in that the palms and other species dispersed occupy multiple ecological niches that in turn provide resources to members of other guilds within the community. It can also be postulated that the hornbill is a significant disperser for taxa with large seeds that may not be able to be handled by other potential dispersers, especially in terms of the hornbill's ability to carry large seeds long distances from parent trees.

\section{Conservation and restoration implications}

Nesting behavior was not a focus of our study, but observations made during the study duration showed no Papuan hornbills to be nesting in areas frequented by humans or significantly disturbed by humans. Papuan hornbills in Halmahera were found nesting in trees in primary and secondary forest only. This indicates, at least anecdotally, that the conservation of protected hornbill nesting sites in undisturbed forest fragments (even if surrounded by mixed land uses) across the landscape may aid in ensuring that hornbills have nesting (i.e., reproductive) areas at the scale needed to ensure not only a minimum viable population but also an ecologically functional population as landscape-scale dispersers. 
Papuan hornbills are most likely common in Halmahera because forested habitat still covers a majority of the island and localities where they can nest/breed, roost, and forage without being hunted out (i.e., extirpated) continue to exist. To the east of Halmahera, a four-month study based in 3 different types of forest habitats of New Britain, Papua New Guinea, included R. plicatus and documented the taxon consuming at least 12 species of plants (Marsden and Pilgrim 2003). Separately, there have been studies on two differing species of hornbills (Penelopides exarhatus and Aceros cassidix) that are native directly to the west of Halmahera, on the larger Indonesian island of Sulawesi, which recorded consumption of between 42 and up to 52 plant species respectively (Walker 2007, Kinnaird and O'Brien 1993). Chapter 2 of this dissertation study demonstrated that Papuan hornbills in Halmahera consume a large quantity of forest plant species (at least 71), and this chapter has demonstrated experimentally that several palm species are being dispersed effectively by hornbills into a variety of habitats and successfully recruiting post-dispersal.

I therefore make the argument that Papuan hornbills are not only effective dispersers of palms but are also likely to be effective dispersers for most, if not all, of the plant species in their diets. This has implications for short-term community assembly as well as longterm evolutionary processes. A dated phylogenetic analysis of all 15 hornbill genera recently proposed that frugivorous hornbills played a meaningful role in the floristic intermixing and evolution of plant taxa from the Indian subcontinent to areas across Southeast Asia and into Melanesia (Viseshakul et al. 2011). Hornbills are important seed 
dispersers in Wallacea, an area highly regarded for its biological diversity and remnant forest tracts, and their role as frugivores aiding ecological and evolutionary processes is becoming increasingly important in the face of logging, habitat conversion and destruction, and hunting pressures (Kinnaird and O'Brien 2007, Chasar et al. 2014, Naniwadekar et al. 2015a).

In addition to maintaining ecological processes through conservation of seed dispersal mutualisms (Howe 2016), researchers have also identified the feasibility of using these biotic interactions as mechanisms for both restoring and monitoring ecosystem functionality (Kaiser-Bunbury et al. 2010). In Halmahera, there is demand for efficient methods for carrying out restorative plantings with native forest species, especially in sites damaged by exploratory mining surveys or pits (M. Abdo, pers. obs.). The results of this study's field experiment combined with relevant literature on the topic suggest a novel avenue that can be explored for future restoration efforts. While long-term data for the Wallacea region remain incomplete, studies elsewhere in Southeast Asia have shown that long-term survival of dispersed saplings at hornbill roost sites tends to be low relative to the original number of dispersed seeds (Kitamura et al. 2008). This study's results demonstrate that germination and early-life seedling survival is relatively high for seeds planted from hornbill-ingested seed traps stationed below roosting sites. A separate study in Sumatra showed that the installation of artificial perches intended to draw hornbills to a restoration area was not successful. Hornbill roost sites in Halmahera may provide a practical source for obtaining viable seed from a diverse array of forest species, 
without expending the effort of searching for reproductive trees, obtaining quantities of their mature diaspores, and removing their pulp manually to aid germination. This method could be experimentally trialled in a future study, as long as strict controls were in place e.g., 1) ensuring that the identification of and activity around roosting sites does not cause any hornbill hunting; and 2) permitting only a percentage of the dispersed seeds collected from roost sites to be harvested for the restoration effort. This could be done on local scales to help rapidly and efficiently establish native forest tree nursery stock.

Alarmingly, during the study period an increase in hornbill hunting was observed. A new effort to assess the population demographics and threats to the Papuan hornbill is warranted. Continued research not only on hornbills but on all Moluccan forest frugivores is also timely and important. Other studies have shown that seed arrival is reduced and recruitment (especially of frugivore diet plant species) is altered in forests where hornbill hunting and logging exists, and in some cases this disproportionately affects large-seeded plants (Naniwadekar et al. 2015a, Sethi and Howe 2009). Given the current lack of information on possible dispersal redundancy for the ecological service Papuan hornbills provide in Halmahera, and evidence that hornbills are being increasingly hunted, urgent measures to ban hunting and protect hornbill populations are imperative. Protection of the Papuan hornbill as an important seed disperser will aid in both maintaining and restoring forests.

The link between forest plant dispersal and regeneration, both within intact forests and in disturbed areas of Halmahera, and the dispersal service provided by hornbills is clear. 
Plant populations, including many forest trees and palms, will suffer if hornbills continue to be extirpated across the island. Palm species that have been shown by this study to be effectively dispersed by hornbills are components of many habitats in the Moluccan islands (M. Abdo, unpublished data), occupy diverse ecological niches, and provide food for other frugivores (Zona and Henderson 1989). Further, some of the very trees for which the Moluccan islands gained their famous moniker as the "Spice islands" are largeseeded trees in the Myristicaceae family that rely on the dispersal service of Papuan hornbills.

\section{Future directions}

Virtually no recent studies on plant-animal interactions from the large and ecologically important island of Halmahera exist. On Halmahera island, in addition to the Papuan hornbill, other potential moderate- to large- bodied frugivores include fruit pigeons (e.g., Ducula spp., Ptilinopus spp.), bats, civets, wild pigs, the Javan deer, cus-cus, and possibly river eels, fish, and rats. Study into the spectrum of seed dispersal and seed predation activities by any of these potential frugivores and gaining insights into guild interactions and their effects on plant community assembly would be viable directions for future work.

Future study of the Papuan hornbills' role in seed dispersal just west of the study area in Papua New Guinea would be beneficial, as some of the world's largest remaining tracts of primary forest remain in this area yet knowledge of plant-animal interactions in Papua 
is lacking. The Papuan hornbill is the only hornbill taxon that is native to the island of Papua New Guinea, and therefore it is likely to be an important seed disperser there.

Two of the palm species studied, Rhopaloblaste ceramica and Saribus rotundifolius, have relatively large diaspores (both typically over $20 \mathrm{~mm}$ in length), with Rhopaloblaste ceramica having a narrower distribution/range and larger fruits. It would be interesting to examine the broader community of potential dispersers of these taxa in order to learn if there is effective dispersal redundancy for either of these species (one might hypothesize that the wider range and slightly smaller diaspores of $S$. rotundifolius indicate likelihood of higher dispersal redundancy than for that of $R$. ceramica). The question of dispersal redundancy, especially amongst large-seeded tropical tree and palm species, has rarely been studied in insular ecologies. That said, in two island-based studies researchers found very low dispersal redundancy (a single effective disperser in both instances) for large-seeded plant species (Wotton and Kelly 2011, McConkey and Drake 2015).

Other dimensions of the dispersal dynamics of hornbills, e.g., temporal variation in hornbills' food species preferences, resource tracking at multiple scales, if there are plant species with disproportionate or obligate reliance on hornbills for dispersal, the recruitment and long-term survival of all dispersed seed species, movement behavior at landscape scale and across fragments, and ecological effects of reduced hornbill abundance caused by logging/hunting are dimensions that have only been examined in a small number of studies (e.g., Kinnaird 1998, Datta 2001, Kitamura et al. 2008, Sethi and Howe 2009, Lenz et al. 2011, Lamperti et al. 2014, Chasar et al. 2014, Mueller et al. 
2014, Naniwedekar et al. 2015a, Naniwedekar 2015b). All of these dimensions and aforementioned studies provide a basis for the development of research questions and methodologies for future studies on Halmahera and beyond.

\section{REFERENCES}

BirdLife International. (2016). Important Bird Areas factsheet: Aketajawe. Downloaded from http://www.birdlife.org on 26/11/2016.

Chasar, A., R.J. Harrigan, K.M. Holbrook, T.V. Dietsch, T.L.Fuller, M. Wikelski, and T.B. Smith. (2014). Spatial and Temporal Patterns of Frugivorous Hornbill Movements in Central Africa and their Implications for Rain Forest Conservation. Biotropica, 46 (6) 763-770.

Clements, J. F., T. S. Schulenberg, M. J. Iliff, D. Roberson, T. A. Fredericks, B. L. Sullivan, and C. L. Wood. (2016). The eBird/Clements checklist of birds of the world: v2016. Downloaded from http://www.birds.cornell.edu/clementschecklist/download/ on 26/11/2016.

Corlett, R. T. (1998). Frugivory and seed dispersal by vertebrates in the oriental (Indomalayan) region. Biological Reviews of the Cambridge Philosophical Society, 73(04), 413-448.

Cramer, J. M., Mesquita, R. C., \& Williamson, G. B. (2007). Forest fragmentation differentially affects seed dispersal of large and small-seeded tropical trees. Biological Conservation, 137(3), 415-423.

Datta, A. (2001). An ecological study of sympatric hornbills and fruiting patterns in a tropical forest in Arunachal Pradesh. Rajkot, Saurashtra University. PhD, 265.

de Almeida, L. B., \& Galetti, M. (2007). Seed dispersal and spatial distribution of Attalea geraensis (Arecaceae) in two remnants of cerrado in southeastern Brazil. Acta Oecologica, 32(2), 180-187.

Dennis, A.J., Schupp, E.W., Green, R.J., \& Westcott, D.A. (2007). Seed Dispersal: Theory and Its Application in a Changing World. CAB International, Wallingford, UK. 
Donatti, C., Galetti, M., Pizo, M., Guimaraes Jr, P., \& Jordano, P. (2007). Living in the land of ghosts: Fruit traits and the importance of large mammals as seed dispersers in the pantanal, Brazil. in Seed Dispersal: Theory and its Application in a Changing World Dennis AJ, Green RJ, Schupp EW, Westcott DA, 104-123.

Dransfield, John. (1978). "Growth forms of rain forest palms," 247-268 in Tropical trees as living systems, Tomlinson, P.B. and MH Zimmermann. Cambridge Univ. Press, New York.

Fadini, R. F., Fleury, M., Donatti, C. I., \& Galetti, M. (2009). Effects of frugivore impoverishment and seed predators on the recruitment of a keystone palm. Acta Oecologica, 35(2), 188-196.

Gautier-Hion, A., Duplantier, J., Quris, R., Feer, F., Sourd, C., Decoux, J., . . .

Hecketsweiler, P. (1985). Fruit characters as a basis of fruit choice and seed dispersal in a tropical forest vertebrate community. Oecologia, 65(3), 324-337.

Govaerts, R., Dransfield, J., Zona, S.F, Hodel, D.R. \& Henderson, A. (2016). World Checklist of Arecaceae. Facilitated by the Royal Botanic Gardens, Kew. Published on the Internet; http://apps.kew.org/wcsp/ Retrieved 2016-11-24

Guimarães, P. R., Lopes, P. F., Lyra, M. L., \& Muriel, A. P. (2005). Fleshy pulp enhances the location of Syagrus romanzoffiana (Arecaceae) fruits by seed-dispersing rodents in an atlantic forest in south-eastern brazil. Journal of Tropical Ecology, 21(01), 109-112.

Holbrook, K. M., Smith, T. B., \& Hardesty, B. D. (2002). Implications of long-distance movements of frugivorous rain forest hornbills. Ecography, 25(6), 745-749.

Howe, H. F. (2016). Making dispersal syndromes and networks useful in tropical conservation and restoration. Global Ecology and Conservation 6: 152-178.

Howe, H. F. (2014). Diversity Storage: Implications for tropical conservation and restoration. Global Ecology and Conservation 2: 349-358.

Howe, H.F. (1986). Seed dispersal by fruit-eating birds and mammals. Seed Dispersal 123:189.

Howe, H. F. (1984). Implications of seed dispersal by animals for tropical reserve management. Biological Conservation 30: 261-281.

Howe, H.F., and Smallwood, J. (1982). Ecology of seed dispersal. Annual Review of Ecology, Evolution, and Systematics 13: 201-228. 
Kaiser-Bunbury, C.N., Traveset, A., and Hansen, D.M. (2010). Conservation and restoration of plant-animal mutualisms on oceanic islands. Perspectives in Plant Ecology, Evolution and Systematics 12: 131-143.

Kinnaird, M. F. and O'Brien, T. G. (2007). The ecology and conservation of Asian hornbills. University of Chicago Press, Chicago, Illinois

Kinnaird, M.F. (1998). Evidence for effective seed dispersal by the Sulawesi redknobbed hornbill, Aceros cassidix. Biotropica 30, 50-55.

Kinnaird, M.F., T.G. O’Brien, and S. Suryadi. (1996.) Population fluctuation in Sulawesi red-knobbed hornbills: Tracking figs in space and time. Auk 113: 431440.

Kitamura, S. (2011). Characteristics of hornbill-dispersed fruits in lowland dipterocarp forests of southern Thailand. The Raffles Bulletin of Zoology, (24), 137-147.

Kitamura, S., Yumoto, T., Noma, N., Chuailua, P., Maruhashi, T., Wohandee, P., \& Poonswad, P. (2008). Aggregated seed dispersal by wreathed hornbills at a roost site in a moist evergreen forest of Thailand. Ecological Research, 23(6), 943-952.

Kitamura S., Yumoto T, Poonswad P, Noma N, Chuailua P, Plongmai K, Maruhashi T, Suckasam C. (2004). Pattern and impact of hornbill seed dispersal at nest trees in a moist ever- green forest in Thailand. Journal of Tropical Ecology 20:545-553

Kitamura, S., Yumoto, T., Poonswad, P., Chuailua, P., Plongmai, K., Maruhashi, T., \& Noma, N. (2002). Interactions between fleshy fruits and frugivores in a tropical seasonal forest in Thailand. Oecologia, 133(4), 559-572.

Lenz, J., Fiedler, W., Caprano, T., Friedrichs, W., Gaese, B. H., Wikelski, M., \& BohningGaese, K. (2011). Seed-dispersal distributions by trumpeter hornbills in fragmented landscapes. Proceedings. Biological Sciences / the Royal Society, 278(1716), 2257-2264.

Markl, J. S., Schleuning, M., Forget, P. M., Jordano, P., Lambert, J. E., Traveset, A., Böhning-Gaese, K. (2012). Meta-analysis of the effects of human disturbance on seed dispersal by animals. Conservation Biology, 26(6), 1072-1081.

McConkey, K. R., Prasad, S., Corlett, R. T., Campos-Arceiz, A., Brodie, J. F., Rogers, H., \& Santamaria, L. (2012). Seed dispersal in changing landscapes. Biological

Conservation, 146(1), 1-13.

Mueller, T., J. Lenz, T. Caprano, W. Fielder, K. Boehning-Gaese. (2014.) Large frugivorous birds facilitate functional connectivity of fragmented landscapes. Journal of Applied Ecology 51: 684692. 
Myers, N., Mittermeier, R. A., Mittermeier, C. G., Da Fonseca, G. A., \& Kent, J. (2000). Biodiversity hotspots for conservation priorities. Nature, 403 (6772), 853-858.

Naniwadekar, R., U. Shukla, K. Isvaran, A. Datta. (2015a) Reduced hornbill abundance associated with low seed arrival and altered recruitment in a hunted and logged tropical forest. PLoS ONE 10(3): e0120062. doi:10.1371/journal.pone.0120062

Naniwadekar, R., C. Mishra, A. Datta. (2015b). Fruit resource tracking by hornbill species at multiple scales in a tropical forest in India. Journal of Tropical Ecology, doi: $10.1017 / \mathrm{S} 0266467415000449$

Santhoshkumar, E. and P. Balasubramanian. (2011). Seed dispersal by the Indian grey hornbill Ocyceros birostris in Eastern Ghats, India. Ecotropica 17: 71-77.

Schupp, E. W., Jordano, P., \& Gómez, J. M. (2010). Seed dispersal effectiveness revisited: A conceptual review. New Phytologist, 188(2), 333-353.

Sethi, P., \& Howe, H. F. (2009). Recruitment of Hornbill-Dispersed trees in hunted and logged forests of the indian eastern himalaya. Conservation Biology, 23(3), 710-718.

Suparan, P., R.A.C. Dam, S. van der Kaars, \& T.E. Wong. (2001). Late Quaternary tropical lowland environments on Halmahera, Indonesia. Palaeogeography, Palaeoclimatology, Palaeoecology 171: 229-258

Tiffney, B.H. (2004). Vertebrate dispersal of seed plants through time. Annual Review of Ecology, Evolution, and Systematics 35, 1e29.

Viseshakul N, Charoennitikul W, Kitamura S, Kemp AC, Thong-aree S, Surapunpitak Y, Poonswad P, Ponglikitmongkol M. (2011). A phylogeny of frugivorous hornbills linked to the evolution of Indian plants within Asian rainforests. Journal of Evolutionary Biology 24:1533-1545.

Wang, B. C., \& Smith, T. B. (2002). Closing the seed dispersal loop. Trends in Ecology \& Evolution, 17(8), 379-386.

Webb, C. O., \& Peart, D. R. (2001). High seed dispersal rates in faunally intact tropical rain forest: Theoretical and conservation implications. Ecology Letters, 4(5), 491-499.

Wheelwright, N.T., (1985). Fruit size, gape width, and the diets of fruit-eating birds. Ecology 66: 808-818.

Whittaker, R.J. and Jones, S.H. (1994). The role of frugivorous bats and birds in the rebuilding of a tropical forest ecosystem, Krakatau, Indonesia. Journal of Biogeography $21,245-258$. 
Zona, S., and Henderson, A. (1989). A review of animal-mediated seed dispersal of palms. Selbyana 11: 6-21.

\section{FIGURES AND TABLES}

Figure 1: Map illustrating the Wallacea Biodiversity hotspot (Indonesia) containing the Moluccan islands, highlighting Halmahera island and its Aketajawe-Lolobata National Park

Figure 2: Diagram illustrating planting design of the in-situ palm seed fate experiment carried out at four habitat types on Halmahera island, Indonesia

Figure 3: Comparison of mean germination of three palm (Arecaceae) species experiencing three different diaspore treatments, in a nursery setting.

Figure 4: Germination results of three seed treatments of Pigafetta filaris planted at four different habitat types on Halmahera island, Indonesia.

Figure 5: Recruitment results of three seed treatments of Pigafetta filaris planted at four different habitat types on Halmahera island, Indonesia.

Figure 6: Germination results of three seed treatments of Rhopaloblaste ceramica planted at four different habitat types on Halmahera island, Indonesia.

Figure 7: Recruitment results of three seed treatments of Rhopaloblaste ceramica planted at four different habitat types on Halmahera island, Indonesia.

Figure 8: Germination results of three seed treatments of Saribus rotundifolius planted at four different habitat types on Halmahera island, Indonesia.

Figure 9: Recruitment results of three seed treatments of Saribus rotundifolius planted at four different habitat types on Halmahera island, Indonesia.

Figure 10: Habitats of Halmahera, Indonesia, including habitats studied over the course of my dissertation and used in the in-situ experiment on seed fates in three native palm (Arecaceae) species

Table 1: List of palm species ingested by the Papuan hornbill at three study sites in Halmahera island, Indonesia 
Figure 1: Map illustrating the Wallacea Biodiversity hotspot (Indonesia) containing the Moluccan islands, highlighting Halmahera island and its Aketajawe-Lolobata National Park

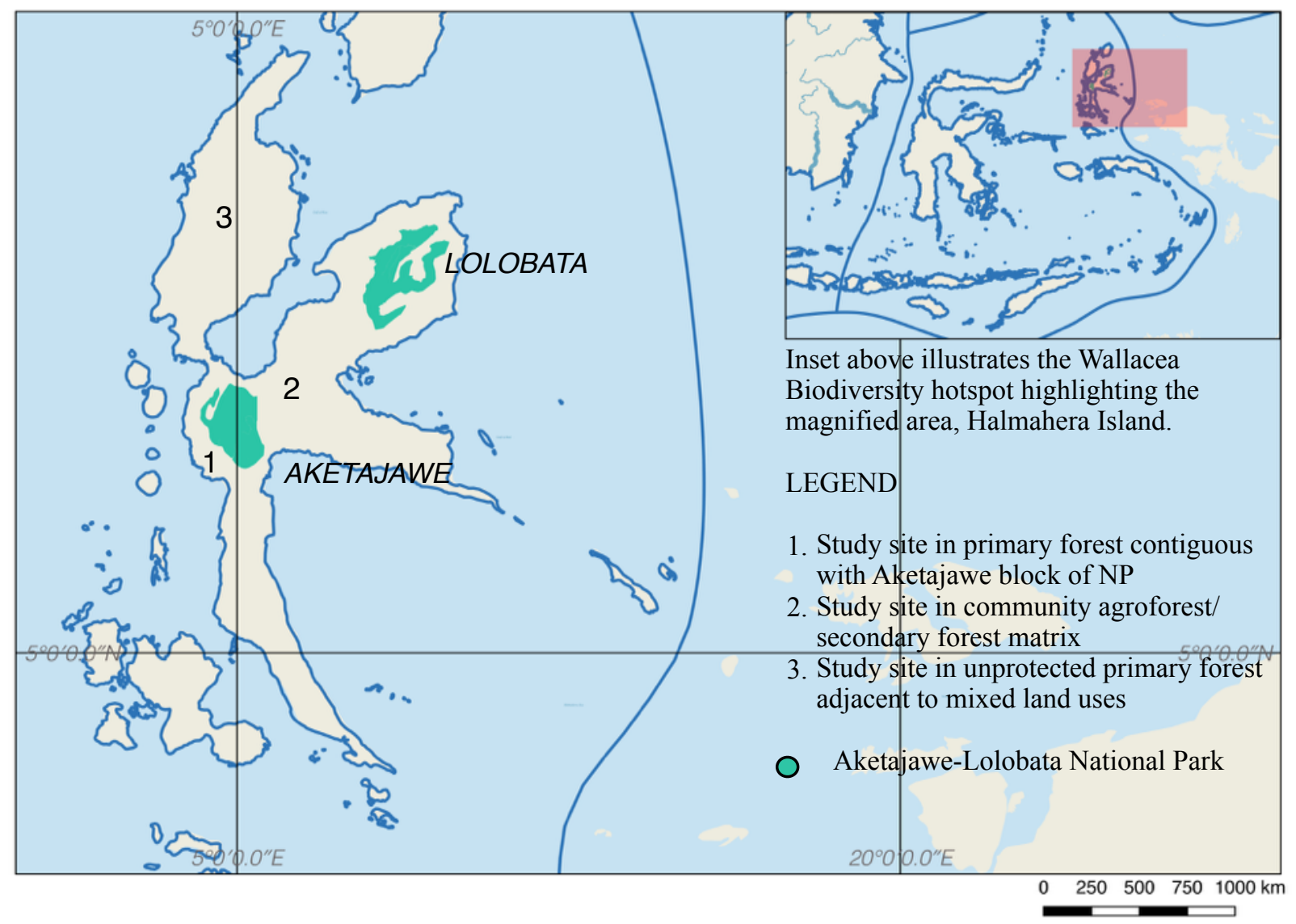


Fig. 2: Diagram illustrating planting design of the in-situ palm seed fate experiment carried out at four habitat types on Halmahera island, Indonesia.

(P1 = palm species \#1, T1 = seed treatment \#1, and so forth for all three palm species and all three treatments. Dashed lines indicate separate zones for each palm species. Dotted lines indicate separate zones for each treatment. Outline mimics tree canopy.)

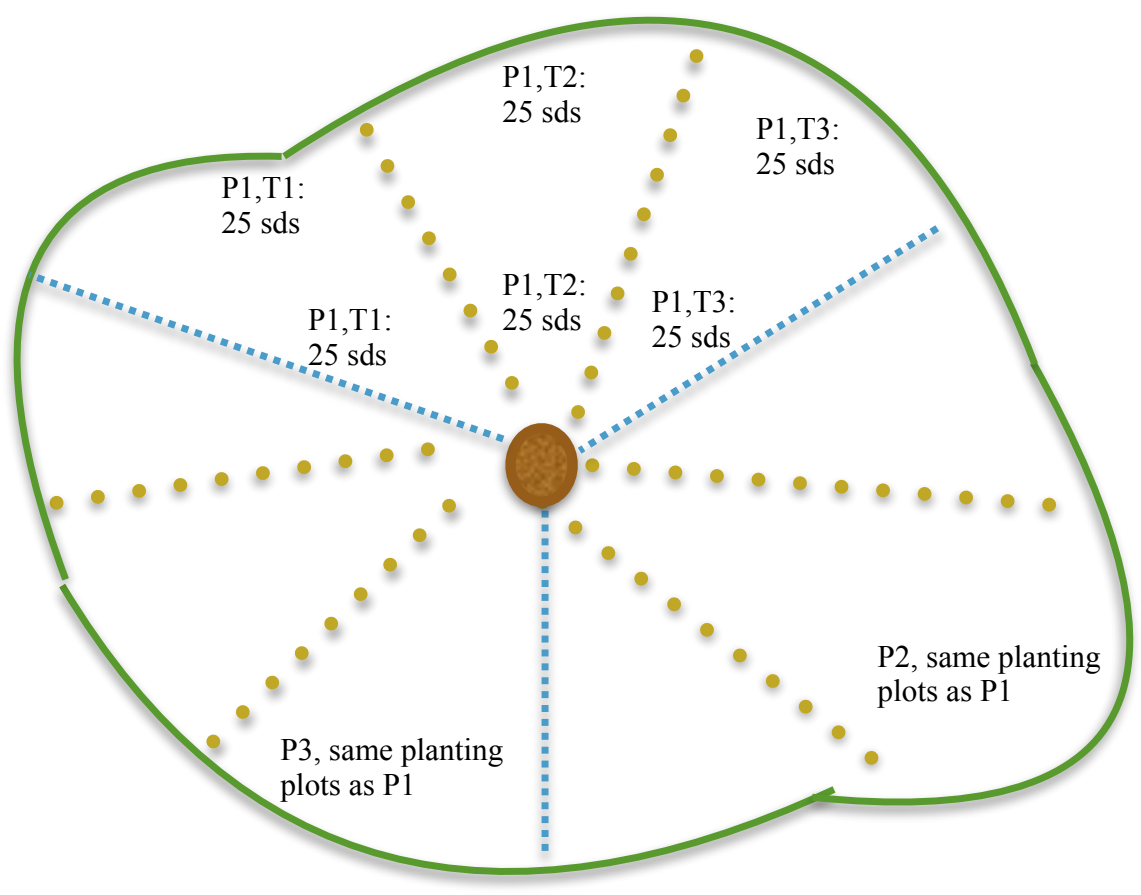


Fig. 3: Comparison of mean germination of three palm (Arecaceae) species experiencing three different diaspore treatments, in a nursery setting. (Error bars indicate standard deviations.)

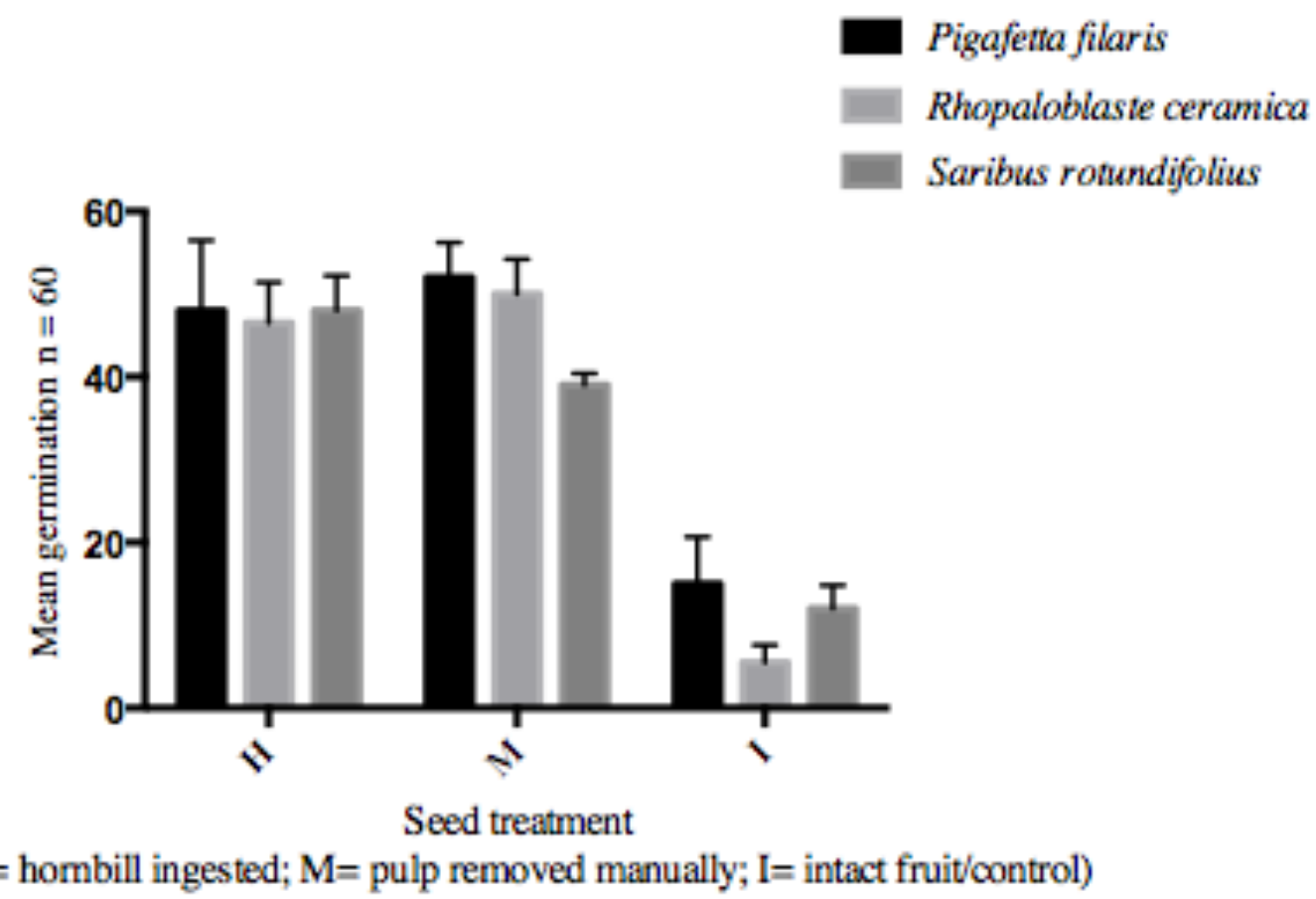


Figure 4: Germination results of three seed treatments of Pigafetta filaris planted at four different habitat types on Halmahera island, Indonesia. (Error bars indicate standard deviations.)

Pigafetta filaris cumulative germination after 10 weeks



Treatments of palm seed across habitats

( $\mathrm{H}=$ hornbill ingested, $\mathrm{M}=$ pulp removed manually, $\mathrm{I}=$ intact fruits/control) 
Figure 5: Recruitment results of three seed treatments of Pigafetta filaris planted at four different habitat types on Halmahera island, Indonesia.

Pigafetta filaris hornbill ingested treatment: recruitment across time at 4 different habitats



Pigafetta filaris pulp removed manually treatment: recruitment across time at 4 different habitats

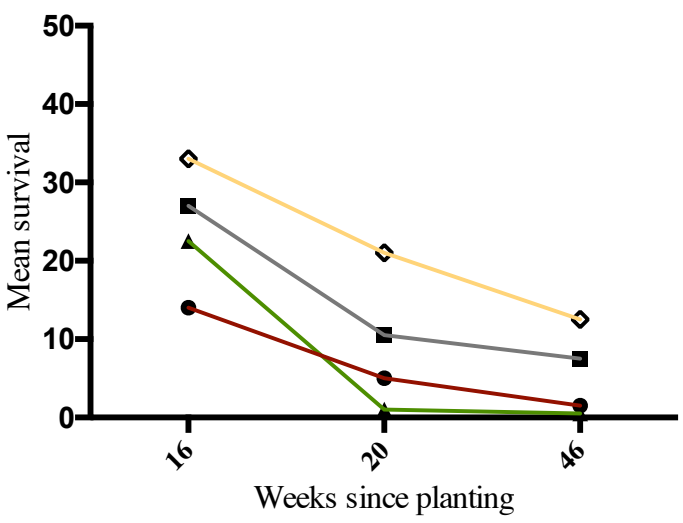
$\multimap$ Parent Palm
- Roost
$\leftarrow$ Primary Forest
$\diamond$ Disturbed

Pigafetta filaris control treatment: recruitment across time at 4 different habitats

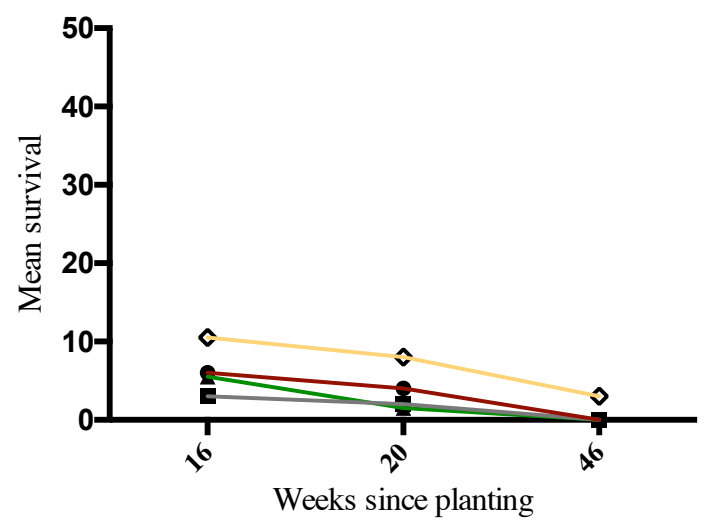


Figure 6: Germination results of three seed treatments of Rhopaloblaste ceramica planted at four different habitat types on Halmahera island, Indonesia. (Error bars indicate standard deviations.)

Rhopaloblaste ceramica cumulative germination after 10 weeks

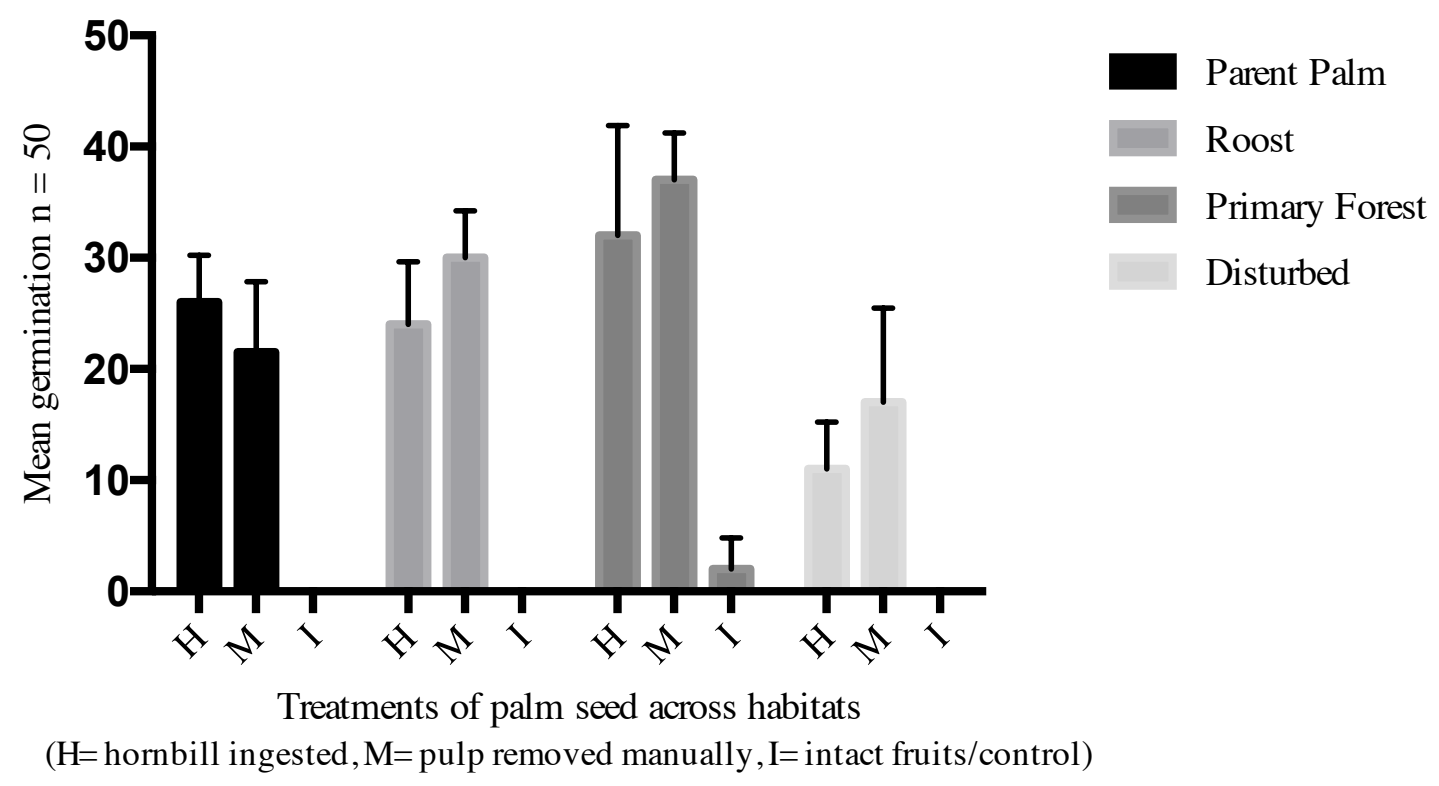


Figure 7: Recruitment results of three seed treatments of Rhopaloblaste ceramica planted at four different habitat types on Halmahera island, Indonesia.

Rhopaloblaste ceramica hornbill ingested treatment: recruitment across time at 4 different habitats



Rhopaloblaste ceramica pulp removed manually treatment: recruitment across time at 4 different habitats

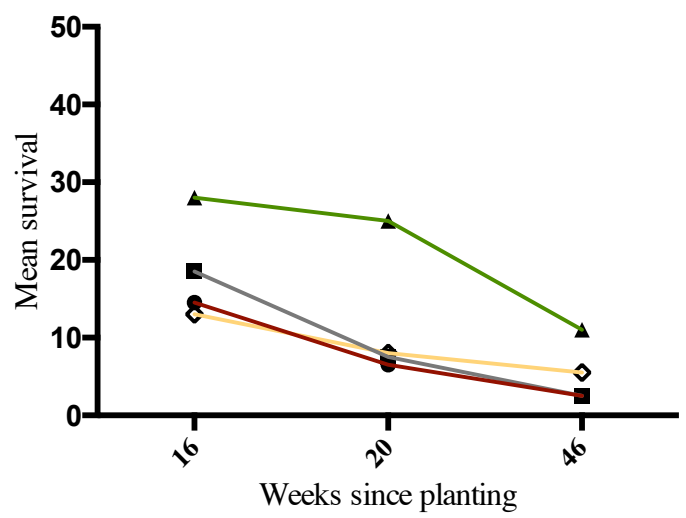
$\rightarrow$ Parent Palm
$\rightarrow$ Roost
$\leftarrow$ Primary Forest
$\diamond$ Disturbed

Rhopaloblaste ceramica control (intact) treatment: recruitment across time at 4 different habitats

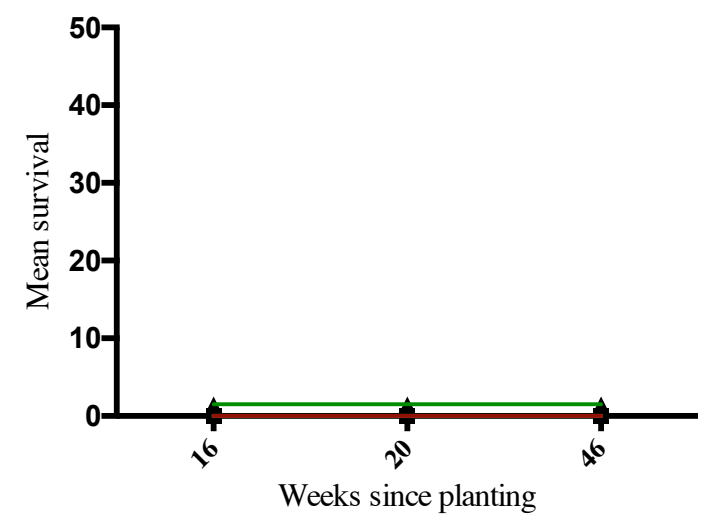
$\rightarrow$ Parent Palm
- Roost
$\leftarrow$ Primary Forest
$\diamond$ Disturbed 
Figure 8: Germination results of three seed treatments of Saribus rotundifolius planted at four different habitat types on Halmahera island, Indonesia. (Error bars indicate standard deviations.)

Saribus rotundifolius cumulative germination after 10 weeks




Figure 9: Recruitment results of three seed treatments of Saribus rotundifolius planted at four different habitat types on Halmahera island, Indonesia.

Saribus rotundifolius hornbill ingested treatment: recruitment across time at 4 different habitats



Saribus rotundifolius pulp removed manually treatment: recruitment across time at 4 different habitats

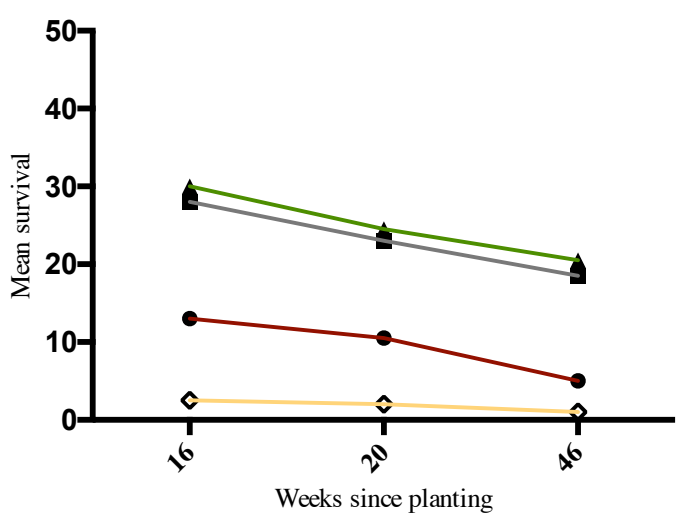

- Parent Palm
- Roost
$\leftarrow$ Primary Forest
$\bullet$ Disturbed

Saribus rotundifolius control treatment: recruitment across time at 4 different habitats

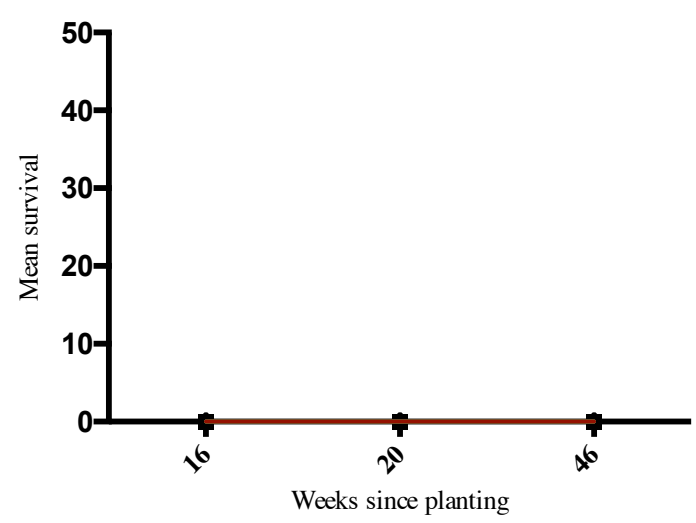


Figure 10: Habitats of Halmahera, Indonesia, including habitats studied over the course of my dissertation and used in the in-situ experiment on seed fates in three native palm (Arecaceae) species


Left (top \& bottom), landscape and close-up view of primary forest habitat; Left (middle), two disturbed habitats; Right (top), Papuan hornbill nesting tree; Right (bottom), Pigafetta filaris, young stand of palms 
Table 1. List of palm species ingested by the Papuan hornbill at three study sites in Halmahera island, Indonesia

\begin{tabular}{|c|c|c|c|c|}
\hline Family & Scientific name & $\begin{array}{l}\text { Vernacular } \\
\text { name(s) }\end{array}$ & $\begin{array}{l}\text { Fruit size } \\
\text { class (in mm) }\end{array}$ & $\begin{array}{l}* \text { Habitat } \\
\text { occurrences }\end{array}$ \\
\hline Arecaceae & Areca vestiaria Giseke & Areca palm & $10-19$ & 1,3 \\
\hline Arecaceae & $\begin{array}{l}\text { Arenga pinnata (Wurmb) } \\
\text { Merr. }\end{array}$ & $\begin{array}{l}\text { Sugar palm, } \\
\text { Aren }\end{array}$ & $20-29$ & $1,2,3$ \\
\hline Arecaceae & $\begin{array}{l}\text { Calamus } \mathrm{L} . \\
\text { sp. A }\end{array}$ & Rattan, rotan & $10-19$ (est.) & 1 \\
\hline Arecaceae & $\begin{array}{l}\text { Calamus L. } \\
\text { sp. B }\end{array}$ & Rattan, rotan & $<10$ (est.) & 1 \\
\hline Arecaceae & $\begin{array}{l}\text { Calamus L. } \\
\text { sp. C }\end{array}$ & Rattan, rotan & $10-19$ (est.) & 3 \\
\hline Arecaceae & Caryota rumphiana Mart. & Fishtail palm & $10-19$ & $1,2,3$ \\
\hline Arecaceae & $\begin{array}{l}\text { Pigafetta filaris (Giseke) } \\
\text { Becc. }\end{array}$ & & $<10$ & $1,2,3$ \\
\hline Arecaceae & $\begin{array}{l}\text { Pinanga rumphiana (Mart.) } \\
\text { J.Dransf. \& Govaerts }\end{array}$ & Palem Pinang & $<10$ & $1,2,3$ \\
\hline Arecaceae & $\begin{array}{l}\text { Rhopaloblaste ceramica } \\
\text { (Miq.) Burrett }\end{array}$ & Gelubing & $\begin{array}{l}\text { usually } 30- \\
39 \mathrm{~mm}, \\
\text { sometimes } \\
\text { smaller }\end{array}$ & $1,2,3$ \\
\hline Arecaceae & $\begin{array}{l}\text { Saribus rotundifolius (Lam.) } \\
\text { Blume }\end{array}$ & Palem raja & $\begin{array}{l}\text { usually } 20 \text { - } \\
\text { 29, sometimes } \\
\text { larger }>30 \mathrm{~mm}\end{array}$ & 1,3 \\
\hline
\end{tabular}

*Habitat occurrences are represented by 1: primary forest contiguous with NP; 2 : community agroforest/ secondary forest; 3 : primary forest in unprotected mixed habitat matrix 


\section{CONCLUSION}

My dissertation research has resulted in a significant increase in scientific knowledge of the ecology of Halmahera, Maluku's largest island and a key repository for Wallacean biodiversity. By utilizing a combination of studies focusing on natural history, field exploration, and ecological experimentation, my research findings contribute to the fields of botany and ecology while also providing management recommendations relevant to the conservation of Halmahera's only National Park. My dissertation contributed the first regional study of dispersal syndromes of Moluccan tree and palm species, the first experimental study of plant-animal interactions and seed dispersal ecology on Halmahera island, and a field study of palm distributions within Halmahera's National Park. Results of the studies have provided insights into the importance of frugivores' seed dispersal service to the assembly and recovery of forests in Wallacea, the diversity of plants dispersed by Papuan hornbills, and the distribution and biogeography of Moluccan palms.

Chapter I explored the palms of the Moluccan islands through field-, herbarium-, and literature- based studies. There are twenty-two genera of palms in Maluku that contain a total of forty-seven palm taxa. Nearly half of the palms (47\%) of Maluku are endemic to the region: twenty-two palm taxa have been recorded only on islands within Maluku. As with other areas in Indonesia and throughout Malesia, most of the palm diversity of Maluku is found in the tribes Areceae and Calameae (Govaerts et al. 2016); combined they contain about $77 \%$ of the species diversity of Maluku. The results of herbarium specimen collections are presented within a preliminary list of palms for Halmahera's 
Aketajawe-Lolobata National Park, and contextualized in a review of regional palm biogeography. On the basis of the field surveys I conducted in the Park, I estimated that at least twenty-seven species of palms are extant in the NP. Several of the herbarium collections made during field research were noteworthy in that they represent species that were previously poorly known in the wild. Two poorly known endemic Hydriastele species (Baker and Loo 2004) were found extant in Aketajawe-Lolobata NP: Hydriastele microcarpa and $H$. beguinii. Heterospathe glauca, another endemic also poorly known in the wild (Gardiner et al. 2012) was found in Tidore, Maluku, within a conservation area. A specimen identified tentatively as a variable phenotype of Heterospathe glauca was also collected within the NP on Halmahera. Many of the palms in the NP were observed in only one of the two disjunct Park blocks, with six species found only in Aketajawe and six other palm species found only in Lolobata. Further study is needed to determine if these disjunct occurrences are indicative of incomplete field study, variation in habitat/community, or are biogeographic in origin. A prudent management recommendation is to study the distribution and demography of palms found only in one Park block and monitor them.

Going beyond the study of one plant family, Chapter II examined all the tree and palm taxa of the Moluccan islands in order to infer seed dispersal syndromes for each taxon. Through analyses of over 900 taxa, I summarized the region's floristic diversity and the dispersal modes of regional trees and palms. I found that the majority of the archipelago's trees, and nearly $80 \%$ of the region's endemic species, rely upon the 
dispersal services of frugivores; these results support the widely-held perspective that the majority of tropical tree species are dispersed by frugivores (Howe and Smallwood 1982, Dennis et al. 2007). Further, the findings that an estimated $20 \%$ of all trees in the Moluccan islands and nearly $30 \%$ of the region's endemic species rely almost exclusively upon the dispersal services of large-bodied frugivores (e.g., hornbills, fruit bats, fruit pigeons) are noteworthy. My study also demonstrated that one large-bodied frugivore in particular, the Papuan hornbill, provides dispersal services for over 70 species of tropical trees and palms on Halmahera. Considering the potential distances travelled by Papuan hornbills, the high number of fruits ingested during a tree visitation, the large proportion of hornbill-digested dispersed seeds that successfully germinate, and the diversity of tree and palm species consumed, Papuan hornbills are effective seed dispersers in the Moluccan islands. Hornbills in Halmahera likely play important roles in forest community assembly as well as forest regeneration, dispersing at least $10 \%$ of the island's tree and palm species.

Chapter III focused on the dispersal of palm species by Papuan hornbills. Specifically, I aimed to discover how dispersal by hornbills and varied habitat types influenced the early life history stages of three palms: Pigafetta filaris, Rhopaloblaste ceramica, and Saribus rotundifolius. Although palms are well-known to rely on the dispersal services of frugivores (Zona and Henderson 1989), to date, little is known about hornbills' role in dispersing palm seeds. I found that the Papuan hornbill ingests and disperses at least 10 different species of palms in Halmahera. My nursery-based experiment demonstrated 
that hornbills have a positive effect in enhancing germination rates of palm seeds in comparison to that of palm fruits that had no pulp removed (when environmental factors were otherwise the same across species and treatments). This indicates that Papuan hornbills, and possibly other large-bodied frugivores that are capable of removing the pulp of diaspores prior to seed deposition/dispersal, play an important role in enhancing the germination success of the species they disperse. An important finding of this study is that without frugivore consumption/ingestion resulting in fruit pulp removal, all three palm species experienced significantly lowered or zero germination and recruitment; this finding validates increasing concerns over the impacts of frugivore declines on plant communities (Vanthomme et al. 2010, Wotton and Kelly 2011, Howe 2016).

Dispersal by hornbills also imparted benefits to palms in a range of habitats in Halmahera, in marked contrast to control palm seeds. The results of the experimental field study indicate that some hornbill-dispersed palm seeds do survive to recruit into seedlings almost a year post-dispersal in several different habitats. This is an important contribution as only a couple of studies have investigated hornbill-dispersed seed fates in habitats other than roosting or nesting sites (i.e., Kitamura et al. 2008, Datta 2001), and understanding how dispersal by hornbills (and other large-bodied frugivores) acts as a mechanism contributing to plant community assembly in a variety of habitats is desirable. Howe (2016) has highlighted that understanding seed dispersal dynamics in disturbed habitats adjacent to forests is increasingly important given the declining state of tropical landscapes today, and my study's finding that seeds undergoing dispersal treatment (i.e., 
pulp removal and movement away from the parent plant) have increased survival in disturbed habitats is noteworthy. Observations made during this study of hornbills present and/or perching in all the habitats studied indicates that seed deposition into a range of habitats (including disturbed areas) is feasible in Halmahera. Therefore, frugivores such as Papuan hornbills contribute not only to plant community assembly in primary forests, but also to regeneration and assembly processes in disturbed habitats as they aid in moving plant diaspores into disturbed sites. Frugivorous birds and bats have been shown to aid in rebuilding island plant communities elsewhere in Indonesia (Whittaker and Jones 1994).

\section{REFERENCES}

Baker, W.P. and Loo, A.H.B. (2004). A synopsis of the genus Hydriastele (Arecaceae). Kew Bulletin 59: 61-68.

Datta, A. (2001). An ecological study of sympatric hornbills and fruiting patterns in a tropical forest in Arunachal Pradesh. Rajkot, Saurashtra University. PhD, 265.

Dennis, A.J., Schupp, E.W., Green, R.J., \& Westcott, D.A. (2007). Seed Dispersal: Theory and Its Application in a Changing World. CAB International, Wallingford, UK.

Gardiner, L. M., Dransfield, J., Marcus, J., and Baker, W.J. (2012). Heterospathe barfodii, a new species from Papua New Guinea. Palms 56 (2): 91-100.

Govaerts, R., Dransfield, J., Zona, S.F, Hodel, D.R. \& Henderson, A. (2016). World Checklist of Arecaceae. Facilitated by the Royal Botanic Gardens, Kew. Published on the Internet; http://apps.kew.org/wcsp/ Retrieved 2016-11-24

Howe, H. F. (2016). Making dispersal syndromes and networks useful in tropical conservation and restoration. Global Ecology and Conservation 6: 152-178. 
Howe, H.F., and Smallwood, J. (1982). Ecology of seed dispersal. Annual Review of Ecology, Evolution, and Systematics 13: 201-228.

Kitamura, S., Yumoto, T., Noma, N., Chuailua, P., Maruhashi, T., Wohandee, P., \& Poonswad, P. (2008). Aggregated seed dispersal by wreathed hornbills at a roost site in a moist evergreen forest of Thailand. Ecological Research, 23(6), 943-952.

Vanthomme, H., Bellé, B., \& Forget, P. M. (2010). Bushmeat hunting alters recruitment of large-seeded plant species in central Africa. Biotropica, 42, 672-679.

Whittaker, R.J. and Jones, S.H. (1994). The role of frugivorous bats and birds in the rebuilding of a tropical forest ecosystem, Krakatau, Indonesia. Journal of Biogeography $21,245-258$.

Wotton, D. M., \& Kelly, D. (2011). Frugivore loss limits recruitment of large-seeded trees. Proceedings of the Royal Society of London: Biological Sciences, 278, 3345-3354.

Zona, S., and Henderson, A. (1989). A review of animal-mediated seed dispersal of palms. Selbyana 11: 6-21. 
VITA

MELISSA E. ABDO

Born, Florida, USA

B.A., Environmental Science, Florida International University, Miami, Florida

2007

Award for Outstanding Service to the Environment, Florida International University

2008

M.Sc., Environmental Studies, Florida International University, Miami, Florida

2008

President's Volunteer Service Award, U.S. Council on Service \& Civic Participation

2013

Ph.D Candidate, Florida International University, Miami, Florida

\section{PUBLICATIONS}

Ardi, W. H., Kusuma, Y. W., Lewis, C. L., Risna, R. A., Wiriadinata, H., Abdo, M. E., \& Thomas, D. C. (2014). Studies on Begonia (Begoniaceae) of the Molucca Islands I: Two new species from Halmahera, Indonesia, and an updated description of Begonia holosericea. Reinwardtia 14(1): 19-26.

Oleas, N., Jestrow, B., Calonje, M., Peguero, B., Jiménez, F., Rodríguez-Peña, R., Oviedo, R., Santiago-Valentín, E., Meerow, A.W., Abdo, M. and Maunder, M., (2013). Molecular systematics of threatened seed plant species endemic in the Caribbean Islands. The Botanical Review, 79(4): 528-541.

Maunder, M., Abdo, M., Berazain, R., Clubbe, C., Jiménez, F., Leiva, A., ... \& FranciscoOrtega, J. (2011). The plants of the Caribbean islands: a review of the biogeography, diversity and conservation of a storm-battered biodiversity hotspot, 154-178 in The Biology of Island Floras. Cambridge University Press, London.

Gann, G.D., M.E. Abdo, et. al. Natives For Your Neighborhood. The Institute for Regional Conservation, Miami. 2005-2017. 